\title{
REHABILITATION \\ OF STROKE PATIENTS \\ WITH SENSOR-BASED SYSTEMS
}

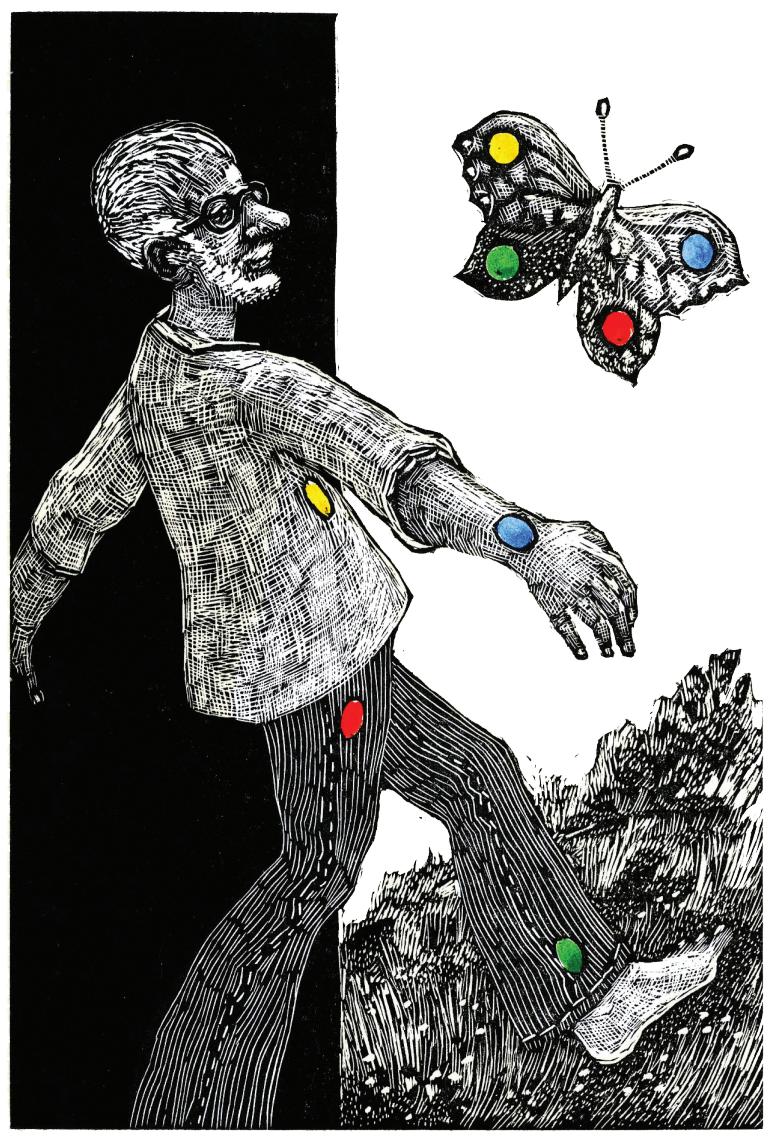

JEREMIA HELD 



\section{REHABILITATION OF STROKE PATIENTS WITH SENSOR-BASED SYSTEMS}

Jeremia Held 
This work was carried out at the:
UNIVERSITY OF TWENTE.
Electrical Engineering, Mathematics and Computer Science, University of Twente, Enschede, the Netherlands

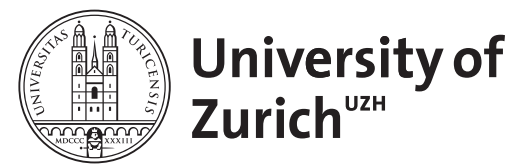
Department of Neurology, University of Zurich and University Hospital Zurich, Zurich, Switzerland

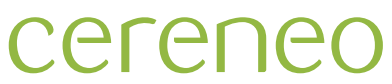
cereneo, Center for Neurology and
CENTER FOR NEUROLOGY
\& REHABILITATION
Rehabilitation, Vitznau, Switzerland.

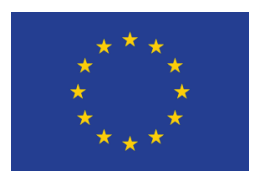

This work was supported by the FP7 project INTERACTION (FP7/ICT project 287351), and project REWIRE (FP7/ICT project 287713), the Swiss Commission for Technology and Innovation (CTI Grant 13612.1) and the P \& K Foundation.

$\begin{array}{ll}\text { Layout } & \text { Renate Siebes | Proefschrift.nu } \\ \text { Printing } & \text { Ridderprint, Ridderkerk } \\ \text { ISBN } & 978-90-365-4708-6 \\ \text { DOI } & 10.3990 / 1.9789036547086 \\ \text { Imprint } & \text { Graphic, drift (2018), Bettina Haller } \\ & \text { Engraving, colour woodcut, coloured }\end{array}$

\section{Copyright $\odot 2018$ Jeremia Philipp Oskar Held}

No parts of this publication may be reproduced or transmitted in any form or by any means, electronic or mechanical, including photocopying, recording, or any information storage or retrieval system, without written permission from the author. 


\title{
REHABILITATION OF STROKE PATIENTS WITH SENSOR-BASED SYSTEMS
}

\author{
DISSERTATION \\ to obtain \\ the degree of doctor at the University of Twente, \\ on the authority of the rector magnificus, \\ Prof.dr. T.T.M. Palstra \\ on account of the decision of the graduation committee, \\ to be publicly defended \\ on Wednesday 13th February 2019 on 12.45 hrs \\ by \\ Jeremia Philipp Oskar Held \\ born on the 10th of July, 1981 \\ in Bielefeld, Germany
}


This dissertation has been approved by:

Promotors:

Prof. dr. ir. P.H. Veltink (University of Twente)

Prof. dr. med. A.R. Luft (University of Zurich)

Co-promotor: $\quad$ Prof. dr. J.H. Buurke (University of Twente) 
Composition of the Graduation Committee:

Chairperson and secretary: $\quad$ Prof. dr. J.N. Kok (University of Twente)

Promotors: $\quad$ Prof. dr. P.H. Veltink (University of Twente)

Prof. dr. A.R. Luft (University Hospital Zurich)

Co-promotor:

Prof. dr. J.H. Buurke (University of Twente)

Members (internal):

Prof. dr. M.M.R. Vollenbroek (University of Twente)

Prof. dr. H. Rietman (University of Twente)

Members (external):

Prof. dr. G. Verheyden (KU Leuven)

Prof. dr. T. Nef (University of Bern)

Dr. J.B.J. Bussmann (Erasmus University Rotterdam)

Paranymphs:

Dr. Janne M. Veerbeek

Albert Eenhoorn 



\section{Contents}

$\begin{array}{lll}\text { Chapter } 1 & \text { General introduction } & 9\end{array}$

$\begin{array}{lll}\text { Chapter } 2 \text { Inertial sensor measurements of upper limb kinematics in } & \mathbf{2 1}\end{array}$

stroke patients in clinic and home environment

Chapter $3 \quad$ Usability evaluation of a vibrotactile feedback system in 41

stroke subjects

Chapter 4 Encouragement-induced real-world upper limb use

after stroke by a tracking and feedback device: a study

protocol for a multi-center, assessor-blinded, randomized

controlled trial

Chapter 5 Self-directed arm therapy at home after stroke with a

sensor-based virtual reality training system

Chapter 6 Does motivation matter in upper limb rehabilitation

after stroke? ArmeoSenso-Reward: Study protocol for a randomized controlled trial.

Chapter 7 Autonomous rehabilitation at stroke patients home for

balance and gait: safety, usability and compliance

of a virtual reality system

Chapter 8 General discussion

Summary

Samenvatting (Summary in Dutch)

Zusammenfassung (Summary in German)

Acknowledgements 



\section{Chapter 1}

General introduction 


\section{STROKE}

A stroke is characterized as a neurological deficit caused by an infarction of the central nervous system in a defined area of a vascular disruption (intracerebral haemorrhage) or a focal ischemic injury based on symptoms persisting longer than 24 hours or until death. ${ }^{1}$ A stroke can occur in any part of the brain. It results in cell death within the nervous system and leads to post-stroke disabilities. Early signs and symptoms of a stroke include the inability to move or feel one side of the body, problems understanding or speaking, and loss of vision on one side. ${ }^{2}$ These impairments depend on the size and localisation of the lesion.

Globally, strokes are the leading cause of long-term disability ${ }^{3}$ and is the number one cause of motor handicap in Europe. ${ }^{4}$ Almost 16,000 people in Switzerland ${ }^{5}$ and 41,000 people in the Netherlands suffer from a stroke each year. ${ }^{6}$ Common, persistent disabilities are upper and lower extremity deficits, cognitive dysfunction, incontinence, and speech problems. ${ }^{7,8}$ Around $80 \%$ of stroke patients experience a unilateral motor deficit, which limits their functionality and engagement in social life, requiring them to use assistance for various activities of daily living (ADLs). ${ }^{9-12}$ To treat post-stroke disabilities, more than two thirds of patients receive rehabilitation services after acute hospitalization. ${ }^{13}$

\section{STROKE REHABILITATION}

Stroke rehabilitation is complex because of the different varieties of brain lesions and diversity of physical and psychological problems. ${ }^{2}$ The rehabilitation process can be distinguished between acute (within the first 24 hours), the early ( 24 hours to 3 months) and late rehabilitation ( 3 to 6 months) as well as rehabilitation in the chronic stages (beyond 6 months). ${ }^{14}$

Stroke rehabilitation is a problem-solving process that aims to decrease the complexity of disabilities and optimize social participation at different stages after a stroke. To tackle the complexity of post-stroke characteristics, it is important to monitor, while assessing the patient, set realistic goals, execute interventions, and re-assess patients' disabilities. ${ }^{8}$

The complexity of stroke can be classified according to the International Classification of Function, Disabilities and Health (ICF). ${ }^{15}$ To classify patients' disabilities and handicaps, a core set for stroke disabilities was developed (Figure 1.1). ${ }^{16}$ This set aims to distinguish problems with stroke subjects in three different categories: functions and structures, activities, and participation. However, the functions and structures categories can be subdivided into capacity, or what a patient can do in a standard environment, and performance, 


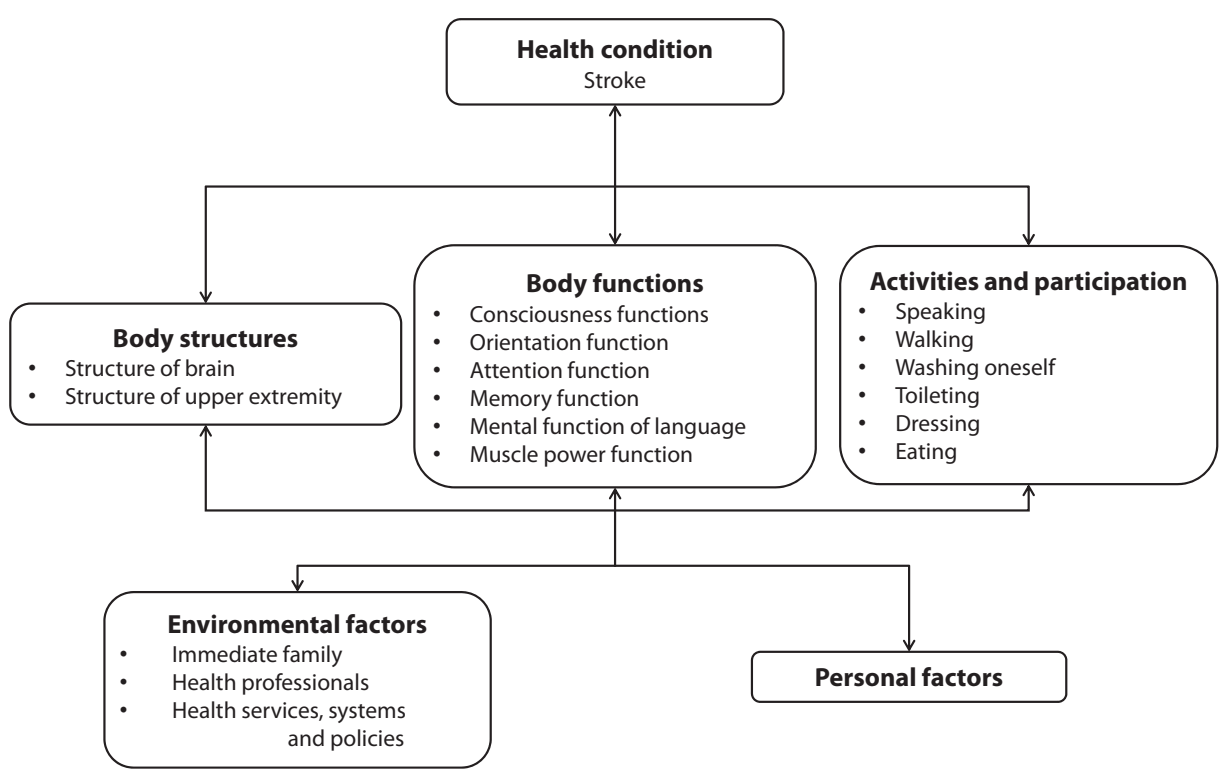

Figure 1.1: Brief ICF Core Set for Stroke. ${ }^{16}$

or what a person actually does in their usual environment. Participation is defined by involvement in daily life.

An important limitation in stroke rehabilitation is described by James Gordon: 'It is easy enough to "facilitate" a certain pattern of movement. What is difficult is to get patients to use that pattern when they are actually carrying out some functional activity. This is the fundamental challenge facing rehabilitation therapists. ${ }^{17}$ To face this challenge, it is important to monitor patients and support stroke rehabilitation interventions after patients are discharged in order to transfer what is taught in clinics over to patients' daily lives and achieve the final goal of independent living at home. ${ }^{18}$ This is also important to prevent a functional decline of ADLs in the first two years. ${ }^{19}$ Based on this knowledge, it is critical to detect a functional decline by performing long-term monitoring after a stroke and planning appropriate stroke rehabilitation interventions.

\section{Monitoring patients after a stroke}

Monitoring patients after a stroke is essential to organizing the rehabilitation process, and the measurement of time-points should be well defined, based on the neural repair process. ${ }^{20}$ Monitoring can be differentiated between laboratory assessments performed in the rehabilitation clinic and assessments in daily life. Laboratory assessments reflect patients' 
best abilities (i.e. capacity; Table 1.1), as they are encouraged by a therapist (e.g. Fugl-Meyer Assessment, Action Research Arm Test, 10 Meter-Walk-Test). Laboratory assessments are also important for identifying and quantifying different levels of function and activity. ${ }^{15}$ To measure what patients do in their daily lives (i.e. performance; Table 1.1), clinicians and researchers traditionally rely on semi-structured interviews. ${ }^{15}$ Movement analysis systems, such as optical tracking systems and sensor-based systems to quantify stroke patients' function, ${ }^{21}$ have been added to stroke rehabilitation guidelines and have been widely used for clinical research in recent years. ${ }^{20}$ These technologies tackle problems with floor and ceiling effects in clinical assessments and allow for more objective measurements of performance. ${ }^{22}$ These measurements are important for reflecting the quality of stroke patients' motor performance during the rehabilitation process. Functional activities can be measured with optical tracking systems (e.g. Qualisis) or movement-sensor systems (e.g. Xsens) Figure 1.2. Optical tracking systems remain restricted to motion capture laboratories and cannot be used in daily life. Sensor-based technologies allow for the continuous monitoring of performance during daily life and can guide the rehabilitation process. ${ }^{23}$

A
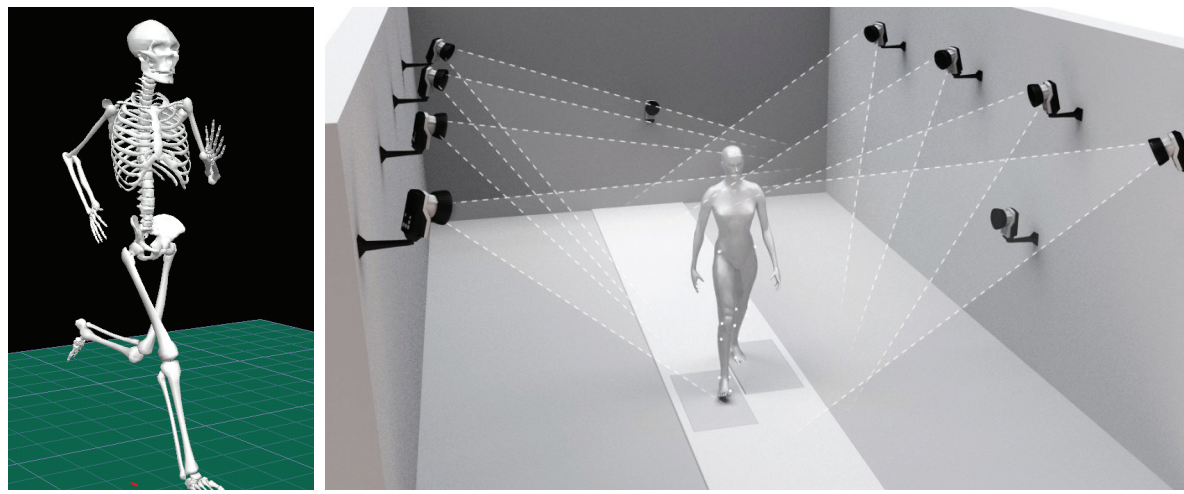

B
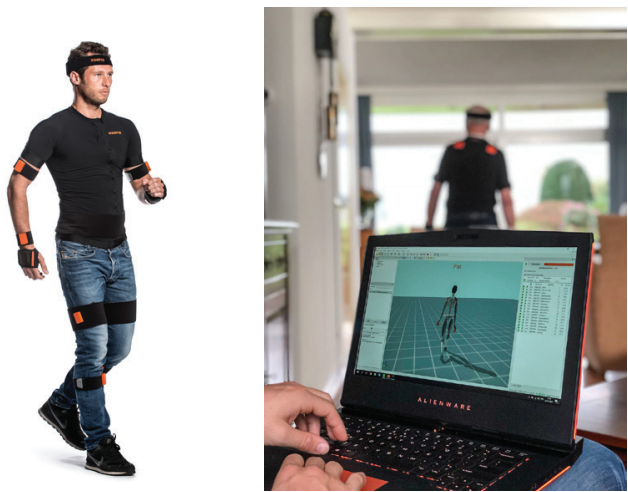

Figure 1.2: A) Optical tracking system - Qualisys; B) Movement-sensor system - Xsens. 
Sensor-based systems (Table 1.1) can help measure physical properties of performance. ${ }^{24-26}$ However, it is unknown how patients' performance, objectively measured by sensor-based systems during daily life, match and complement standard clinical assessments. Stroke rehabilitation interventions intend to improve patients' performance in daily life, but this has never been objectively evaluated. In addition, sensor-based systems can assess patients' performance not only during daily life, but also during therapies, and the therapy can subsequently be adapted based on patients' performances during interventions. ${ }^{27}$

Table 1.1: Definitions

\begin{tabular}{ll}
\hline Term & Definition \\
\hline Sensor-based systems & $\begin{array}{l}\text { Movement sensor technology to monitor or control movements or } \\
\text { objects in an environment }\end{array}$ \\
\hline Performance & $\begin{array}{l}\text { Activities that are performed in daily life without the encouragement } \\
\text { of a therapist; knowledge about what people do in daily life }\end{array}$ \\
\hline Capacity & $\begin{array}{l}\text { Activities that are performed during a predefined task with the } \\
\text { encouragement of an assessor to achieve the best possible task } \\
\text { performance; the maximum potential of what a person can do }\end{array}$ \\
\hline Self-directed therapy & $\begin{array}{l}\text { Therapy where patients perform activities by themselves } \\
\text { Inertial measurement unit (IMU) }\end{array}$ \\
$\begin{array}{l}\text { An electronic device that measures and reports the acceleration, } \\
\text { angular rate, and environmental magnetic field, while being placed } \\
\text { on an object }\end{array}$ \\
\hline
\end{tabular}

\section{Neurorehabilitation stroke intervention}

Neurorehabilitation is effective in increasing stroke patients' independence in ADLs. ${ }^{14}$ Key aspects of effective stroke rehabilitation are intensity, specificity, feedback, and enrichment. ${ }^{14,28}$ It has been shown already that intensity correlates positively with functional outcomes, ${ }^{14,28}$ implying that post-stroke therapy should be highly intensive. ${ }^{28}$ High intensity therapy is easily organized in the first weeks after a stroke in clinical rehabilitation settings. After discharge from the rehabilitation clinic, training at patients' homes and therapy during ADLs are important to prevent functions from deteriorating. ${ }^{29,30}$ However, the delivery of such intensive home therapy in a traditional one-to-one setting requires extensive therapist support, which in practice is not often feasible to implement due to high costs, logistics, and limited human resources. However, traditional, self-reliant home therapy without therapist supervision often suffers from low compliance and patients' lack of motivation to complete the instructed rehabilitative training at the recommended frequency. ${ }^{31}$ To increase rehabilitation intensity, rehabilitation technologies are increasingly important, especially with the use of robotics and virtual reality. ${ }^{32,33}$ Nevertheless, this type of training 
is most often performed in rehabilitation clinics. In recent years, the development of monitoring and intervention technologies has created low-cost tools, such as movementsensors and cameras, to control virtual reality gaming platforms for stroke rehabilitation in patients' homes. Commercial, sensor-based home intervention systems (e.g. Wii (2006, Nintendo Co., Japan), Kinect (Microsoft Inc., USA)) were developed to encourage users to be more physically active. These systems include structured exercise for the upper and lower extremities. Such entertainment systems have been tested with the elderly and with stroke patients, producing results similar to conventional therapy. ${ }^{34,35}$ However, these systems were not designed for patients with neurological disorders, and they do not provide training in tasks that are clinically meaningful to reduce impairments. To deliver specific interventions after discharge from rehabilitation clinics and to enrich the home environment of stroke patients, it is important that sensor-based home interventions are motivating, tailored to patients' impairments, and monitoring the task performed in order to reduce the occurrence of adverse events.

Improvement in learned tasks does not transfer to other trained tasks or activities, such as ADLs. ${ }^{33}$ Therefore, an additional factor of intervention that is important is context specificity. Training is almost always performed in the clinic, and to date, beneficial effects have been shown with capacity measures. However, it is unknown how this training translates to daily life. A combination of sensor-based technology and tailored, patient-specific feedback during daily life might increase patients' abilities to reduce their disabilities.

Specificity alone is not enough for stroke rehabilitation. Reward and feedback during rehabilitation have also been shown to increase the effectiveness of learning new motor tasks. ${ }^{36-39}$ Therefore, the feedback provided should be tailored to individual needs with the goal to increase motivation for, compliance with, and effectiveness of an intervention. Various forms of feedback, like visual, tactile, proprioceptive, or auditory response, during training are known to support the stroke rehabilitation process. ${ }^{40}$

\section{THESIS PROJECTS}

Most sensor-based systems have been developed to monitor and/or encourage physical activity in the general population. They were not designed for use in stroke rehabilitation. While some of these systems have been tested with stroke patients during inpatient rehabilitation, only a few have been used in patients' homes to monitor and/or treat disabilities remaining after discharge. However in recent years, sensor-based systems have been developed in different projects to specifically monitor and treat stroke patients' disabilities. 
This thesis focuses on the use of these sensor-systems that were specifically developed to monitor and treat stroke patients.

The research in this Ph.D. thesis was performed in the framework of several projects:

In the research project 'INTERACTION', a wearable sensor system was developed to monitor stroke patients' quality of movement during performance. This project was funded by the European Union under the $7^{\text {th }}$ Framework Program. International partners were from the Netherlands with the Biomedical Signals and Systems group of the University Twente, Roessingh Research and Development, and Xsens Technologies B.V.; from Switzerland with the University Zurich; and from Italy with Smartex S.r.l. and the University of Pisa. In addition to the monitoring system, a new feedback system, the 'Arm Usage Coach', was designed to motivate stroke patients to use their affected arm more often during daily life performance.

The idea of the Arm Usage Coach was further developed in Swiss national project 'ISEAR' to investigate the effect of rewards on arm use in daily life. The partners involved were the University Hospital Zurich, Rehabilitation Engineering Laboratory of the Swiss Federal Institute of Technology in Zurich, Zurich University of the Arts, FHNW University of Applied Sciences and Arts Northwestern Switzerland, and industrial partner yband therapy AG collaborate.

Furthermore, in Swiss national project, 'ArmeoSenso', a sensor-based system was developed for unsupervised, sensor-based home therapy for upper extremities. The ArmeoSenso was a collaborative project with the University Hospital Zurich, the Rehabilitation Engineering Laboratory of the Swiss Federal Institute of Technology in Zurich, the Balgrist University Hospital, and industrial partner, Hocoma. To further investigate the impact of rewards on stroke rehabilitation intervention, the ArmeoSenso-Reward system was developed.

In European project 'REWIRE' under the $7^{\text {th }}$ Framework Program, a home rehabilitation system was developed to train balance and gait in patients after a stroke. The project partners were the Università degli Studi di Milano; the Ecole Polytechnique Fédérale de Lausanne; the Chancellor, Masters, and Scholars of the University of Oxford; the Università degli Studi di Padova; the Swiss Federal Institute of Technology in Zurich; Ab.Acus Srl; IAVANTE; Fundación Pública Andaluza para el Avance Tecnológico y el Entrenamiento Profesional. Consejería de Salud de Andalucía; Technogym SpA (TECHNO); Fundació Privada Barcelona Digital Centre Tecnològic; the University Hospital Zurich; and the Jožef Stefan Institute Andalusia Health Service-Virgen del Rocío-University Hospital. 


\section{THESIS OBJECTIVES}

The objectives of this thesis are outlined below:

1. To evaluate a sensor-based system that can quantify upper limb activities of stroke patients during the rehabilitation process, in the rehabilitation clinic, and in the home environment.

2. To evaluate the usability and efficacy of sensor-based systems with feedback modalities for stroke rehabilitation interventions.

a. Evaluate how sensor-based systems can be used in daily life to treat stroke patients' disabilities.

b. Evaluate how the provision of rewards by sensor-based systems can influence rehabilitation outcomes in stroke patients?

c. Evaluate how sensor-based systems can be used in patients' home environments without therapists' supervision?

Chapter 2, addressing objective 1, longitudinally to explore the parallels between poststroke, upper limb capacity measured with standard clinical assessments and daily-life performance using IMUs (Table 1.1) during the transition from inpatient rehabilitation to home. These data could be valuable in planning and monitoring rehabilitation therapy when patients are in their home environment.

In Chapter 3 (objective 2a), the usability and acceptance of a vibrotactile feedback system for stroke patients during simulated ADLs are investigated. The Arm Usage Coach aims to train stroke patients in ADLs by monitoring their performance and giving real-time feedback. Based on the results in this chapter, I determine in Chapter 4 (objective 2c) the effects of wearing a wrist-worn, commercially available tracking device. This device provides multimodal feedback on the amount of upper limb use in daily life. The intervention is currently investigated in a randomized controlled trial (RCT), for hemiparetic subjects three months after a stroke. The intervention compares to a control group receiving an identical, sham wrist-device providing no feedback (sham). In Chapter 5 (objective 2b), I investigate the feasibility, safety, and first effects of self-directed home therapy (Table 1.1) using a sensorbased, virtual therapy system (ArmeoSenso). Furthermore, Chapter $\mathbf{6}$ (objective 2b) presents a protocol that describes an RCT to investigate the effect of enhanced feedback and rewards on upper limb outcome measures after a stroke. In addition to upper-extremity stroke rehabilitation, the usage (acceptance and compliance) and safety of the home autonomous therapy system (objective 2a) for balance and gait is investigated in Chapter 7. Finally, Chapter 8 presents the conclusion and discussion as well as future outlook, reflecting the main and sub-goals of this thesis and the work that has been completed. 


\section{REFERENCES}

1. Sacco RL, Kasner SE, Broderick JP, Caplan LR, Connors JJ, Culebras A, Elkind MS, et al. An updated definition of stroke for the 21st century: a statement for healthcare professionals from the American Heart Association/American Stroke Association. Stroke. 2013;44(7):2064-2089.

2. Donnan GA, Fisher M, Macleod M, Davis SM. Stroke. Lancet. 2008;371(9624):1612-1623.

3. Mozaffarian D, Benjamin EJ, Go AS, Arnett DK, Blaha MJ, Cushman M, de Ferranti S, et al. Heart disease and stroke statistics--2015 update: a report from the American Heart Association. Circulation. 2015;131(4):e29-322.

4. Bejot Y, Benatru I, Rouaud O, Fromont A, Besancenot JP, Moreau T, Giroud M. Epidemiology of stroke in Europe: geographic and environmental differences. Journal of the Neurological Sciences. 2007;262(1-2):85-88.

5. Meyer K, Simmet A, Arnold M, Mattle H, Nedeltchev K. Stroke events, and case fatalities in Switzerland based on hospital statistics and cause of death statistics. Swiss Medical Weekly. 2009;139(5-6):65-69.

6. Nederlandse Hartstichting. Beroerte. 2017; https://www.hartstichting.nl/berorte. Accessed 13 November 2017.

7. Lawrence ES, Coshall C, Dundas R, Stewart J, Rudd AG, Howard R, Wolfe CD. Estimates of the prevalence of acute stroke impairments and disability in a multiethnic population. Stroke. 2001;32(6):1279-1284.

8. Langhorne P, Bernhardt J, Kwakkel G. Stroke rehabilitation. Lancet. 2011;377(9778):1693-1702.

9. Langhorne P, Sandercock P, Prasad K. Evidence-based practice for stroke. Lancet Neurology. 2009;8(4):308-309.

10. Wolfe CD, Giroud M, Kolominsky-Rabas P, Dundas R, Lemesle M, Heuschmann P, Rudd A. Variations in stroke incidence and survival in 3 areas of Europe. European Registries of Stroke (EROS) Collaboration. Stroke. 2000;31(9):2074-2079.

11. Lai SM, Studenski S, Duncan PW, Perera S. Persisting consequences of stroke measured by the Stroke Impact Scale. Stroke. 2002;33(7):1840-1844.

12. Johansson A, Mishina E, Ivanov A, Bjorklund A. Activities of daily living among St Petersburg women after mild stroke. Occupational Therapy International. 2007;14(3):170-182.

13. Buntin MB, Colla CH, Deb P, Sood N, Escarce JJ. Medicare spending and outcomes after postacute care for stroke and hip fracture. Medical Care. 2010;48(9):776-784.

14. Veerbeek JM, van Wegen E, van Peppen R, van der Wees PJ, Hendriks E, Rietberg M, Kwakkel $\mathrm{G}$. What is the evidence for physical therapy poststroke? A systematic review and meta-analysis. PLoS One. 2014;9(2):e87987.

15. World Health Organization. International Classification of Functioning, Disability and Health: ICF. World Health Organization; 2001.

16. Geyh S, Cieza A, Schouten J, Dickson H, Frommelt P, Omar Z, Kostanjsek N, et al. ICF Core Sets for stroke. Journal of Rehabilitation Medicine. 2004;36(0):135-141.

17. Gordon J. Assumptions underlying physical therapy intervention: Theoretical and historical perspectives. Movement science: Foundations for Physical therapy in Rehabilitation, ed. J. Carr \& R. Sheppard. 1987.

18. Maclean N, Pound P, Wolfe C, Rudd A. Qualitative analysis of stroke patients' motivation for rehabilitation. British Medical Journal. 2000;321(7268):1051-1054.

19. Wolfe CD, Crichton SL, Heuschmann PU, McKevitt CJ, Toschke AM, Grieve AP, Rudd AG. Estimates of outcomes up to ten years after stroke: analysis from the prospective South London Stroke Register. PLoS Medicine. 2011;8(5):e1001033. 
20. Kwakkel G, Lannin NA, Borschmann K, English C, Ali M, Churilov L, Saposnik G, et al. Standardized measurement of sensorimotor recovery in stroke trials: Consensus-based core recommendations from the stroke recovery and rehabilitation roundtable. Neurorehabil Neural Repair. 2017;31(9):784-792.

21. Reinkensmeyer DJ, Burdet E, Casadio M, Krakauer JW, Kwakkel G, Lang CE, Swinnen SP, et al. Computational neurorehabilitation: modeling plasticity and learning to predict recovery. Journal of NeuroEngineering and Rehabilitation. 2016;13(1):42.

22. Thrane G, Sunnerhagen KS, Persson HC, Opheim A, Alt Murphy M. Kinematic upper extremity performance in people with near or fully recovered sensorimotor function after stroke. Physiotherapy Theory and Practice. 2018:1-11.

23. Schweighofer N, Han CE, Wolf SL, Arbib MA, Winstein CJ. A functional threshold for longterm use of hand and arm function can be determined: Predictions from a computational model and supporting data from the extremity constraint-induced therapy evaluation (EXCITE) trial. Physical Therapy. 2009;89(12):1327-1336.

24. Leuenberger K, Gonzenbach R, Wiedmer E, Luft A, Gassert R. Classification of stair ascent and descent in stroke patients. 2014 11th International Conference on Wearable and Implantable Body Sensor Networks Workshops (Bsn Workshops). 2014:11-16.

25. Moncada-Torres A, Leuenberger K, Gonzenbach R, Luft A, Gassert R. Activity classification based on inertial and barometric pressure sensors at different anatomical locations. Physiological Measurement. 2014;35(7):1245-1263.

26. van Meulen FB, Reenalda J, Buurke JH, Veltink PH. Assessment of daily-life reaching performance after stroke. Annals of Biomedical Engineering. 2015;43(2):478-486.

27. Wittmann F, Lambercy O, Gonzenbach RR, van Raai MA, Hover R, Held J, Starkey ML, et al. Assessment-driven arm therapy at home using an IMU-based virtual reality system. Paper presented at: Rehabilitation Robotics (ICORR), 2015 IEEE International Conference on 2015.

28. Lohse KR, Lang CE, Boyd LA. Is more better? Using metadata to explore dose-response relationships in stroke rehabilitation. Stroke. 2014;45(7):2053-2058.

29. Legg LA, Lewis SR, Schofield-Robinson OJ, Drummond A, Langhorne P. Occupational therapy for adults with problems in activities of daily living after stroke. Cochrane Database of Systematic Reviews. 2017;7:CD003585.

30. Taub E. Movement in nonhuman primates deprived of somatosensory feedback. Exercise and Sport Sciences Reviews. 1976;4:335-374.

31. Lenze EJ, Munin MC, Quear T, Dew MA, Rogers JC, Begley AE, Reynolds CF. Significance of poor patient participation in physical and occupational therapy for functional outcome and length of stay. Archives of Physical Medicine and Rehabilitation. 2004;85(10):1599-1601.

32. Laver KE, Lange B, George S, Deutsch JE, Saposnik G, Crotty M. Virtual reality for stroke rehabilitation. Cochrane Database of Systematic Reviews. 2017;11:CD008349.

33. Veerbeek JM, Langbroek-Amersfoort AC, van Wegen EE, Meskers CG, Kwakkel G. Effects of robot-assisted therapy for the upper limb after stroke. Neurorehabilitation and Neural Repair. 2017;31(2):107-121.

34. Donath L, Rössler R, Faude O. Effects of virtual reality training (Exergaming) compared to alternative exercise training and passive control on standing balance and functional mobility in healthy community-dwelling seniors: A meta-analytical review. Sports Medicine. 2016;46(9): 1293-1309.

35. Bonnechère B, Jansen B, Omelina L, Van Sint Jan S. The use of commercial video games in rehabilitation: a systematic review. International Journal of Rehabilitation Research. 2016;39(4): 277-290.

36. Abe M, Schambra H, Wassermann EM, Luckenbaugh D, Schweighofer N, Cohen LG. Reward improves long-term retention of a motor memory through induction of offline memory gains. Current Biology. 2011;21(7):557-562. 
37. Galea JM, Mallia E, Rothwell J, Diedrichsen J. The dissociable effects of punishment and reward on motor learning. Nature Neuroscience. 2015;18(4):597-602.

38. Wachter T, Lungu OV, Liu T, Willingham DT, Ashe J. Differential effect of reward and punishment on procedural learning. Journal of Neuroscience. 2009;29(2):436-443.

39. Widmer M, Ziegler N, Held J, Luft A, Lutz K. Rewarding feedback promotes motor skill consolidation via striatal activity. Progress in Brain Research. 2016;229:303-323.

40. Magill RA, Anderson DI. Motor learning and control: Concepts and applications. Vol 11: McGraw-Hill New York; 2007. 



\section{Chapter 2}

\section{Inertial sensor measurements of upper limb kinematics in stroke patients in clinic and home environment}

J.P.O. Held, B. Klaassen, A. Eenhoorn, B-J.F. van Beijnum,

J. Buurke, P.H. Veltink, A.R. Luft 


\section{ABSTRACT}

Background Upper limb impairments in stroke patients are usually measured in clinical setting using standard clinical assessment. In addition, kinematic analysis using opto-electronic systems has been used in the laboratory setting to map arm recovery. Such kinematic measurements cannot capture the actual function of the upper extremity in daily life. The aim of this study is to longitudinally explore the complementarity of post-stroke upper limb recovery measured by standard clinical assessments and daily-life recorded kinematics.

Methods The study was designed as an observational, single-group study to evaluate rehabilitation progress in a clinical and home environment, with a full-body sensor system in stroke patients. Kinematic data were recorded with a full-body motion capture suit during clinical assessment and self-directed activities of daily living. The measurements were performed at three time points for three hours: (1) two weeks before discharge of the rehabilitation clinic, (2) right after discharge, and (3) four weeks after discharge. The kinematic analysis of reaching movements uses the position and orientation of each body segment to derive the joint angles. Newly developed metrics for classifying activity and quality of upper extremity movement were applied.

Results The data of four stroke patients (three mildly impaired, one sever impaired) were included in this study. The arm motor function assessment improved during the inpatient rehabilitation, but declined in the first four weeks after discharge. A change in the data (kinematics and new metrics) from the daily-life recording was seen in in all patients. Despite this worsening patients increased the number of reaches they performed during daily-life in their home environment.

Conclusions It is feasible to measure arm kinematics using Inertial Measurement Unit sensors during daily-life in stroke patients at the different stages of rehabilitation. Our results from the daily-life recordings complemented the data from the clinical assessments and illustrate the potential to identify stroke patient characteristics, based on kinematics, reaching counts, and work area.

Trial registration https://clinicaltrials.gov, identifier: NCT02118363. 


\section{INTRODUCTION}

Stroke is the third most common cause of disability worldwide. ${ }^{1}$ After stroke, approximately $50 \%$ of all patients have long-term impairments of upper limb motor function. ${ }^{2}$ These impairments and activities are usually measured in the laboratory with standard clinical assessments such as the Fugl-Meyer Assessment - Upper Extremity subscale (FMA-UE) ${ }^{3}$ and Action Research Arm Test (ARAT). ${ }^{4}$ In the past decade, kinematic analysis of the upper extremity using opto-electronic systems in a clinical setting, ${ }^{5-9}$ has been applied as well to evaluate upper limb motor recovery after stroke. ${ }^{10}$ However, these clinical assessments reflect the patients' best abilities as they are encouraged by an assessor. This test situation does not reflect daily-life upper limb use. ${ }^{11}$

In stroke clinical trials, acceleration sensors have been used to measure the patient armactivities in real world. ${ }^{12}$ Although accelerometer sensors can be used to measure movements in the sagittal plane,${ }^{13}$ they cannot provide information regarding three-dimensional (3D) movements of the upper limb. To measure movement quality kinematic metrics from optical motion capture systems quantify the patients' motor abilities on a body function level but remain restricted to a motion capture laboratory and cannot be used in daily life. New technologies such as wearable inertial measurement units (IMUs) make it possible to quantify upper limb motor function in daily-life. ${ }^{14-16}$ IMUs are able to measure movement kinematics without being restricted to certain location. ${ }^{17}$ The application of IMUs in a laboratory setting, has been compared with standard clinical assessments and showed a good correlation to clinical assessments (e.g., FMA-UE) and short simulated daily-life tasks. ${ }^{16}$ This study indicated that achievements during rehabilitation are incompletely implemented in daily-life. ${ }^{18}$

New technologies, with the possibility to continuously perform daily-life monitoring of functional activities in real life, can monitor response to a new therapy, guide recovery, ${ }^{19}$ and may be valuable tools to measure outcomes in clinical trials. For patients who need continuing training after inpatient rehabilitation, it is important to monitor progress and deterioration.

So far it was not possible to study upper limb motor recovery during daily-life in terms of kinematics at different stages after inpatient stroke rehabilitation. The development of new sensor technology made it possible to detect movement kinematics in stroke patients. ${ }^{18}$ 


\section{Aim of the study}

The aim is to longitudinally explore the complementary between post-stroke upper limb recovery measured with standard clinical assessments and daily-life kinematic recordings using IMUs during the transition from inpatient rehabilitation to home. These data could be valuable in planning and monitoring outpatient rehabilitation therapy. ${ }^{20,21}$

\section{METHODS AND MATERIALS}

\section{Study design}

The study was designed as an observational, single-group study to evaluate rehabilitation progress (over six weeks) in a clinical and home environment, with a full-body IMU system in stroke patients (Figure 2.1). Stroke subjects with a first-ever ischemic stroke were admitted to cereneo - Center for Neurology and Rehabilitation, Vitznau, Switzerland. Inclusion criteria were (I) age between 35 and 80 years of age, (II) a hemiparesis as a result of a single unilateral stroke, (III) able to lift their effected arm against gravity and (IV) to walk 10 meters without supervision. Exclusion criteria were the inability to understand questionnaires and inability to perform given instructions. Patients were recruited between January 2014 and January 2015.

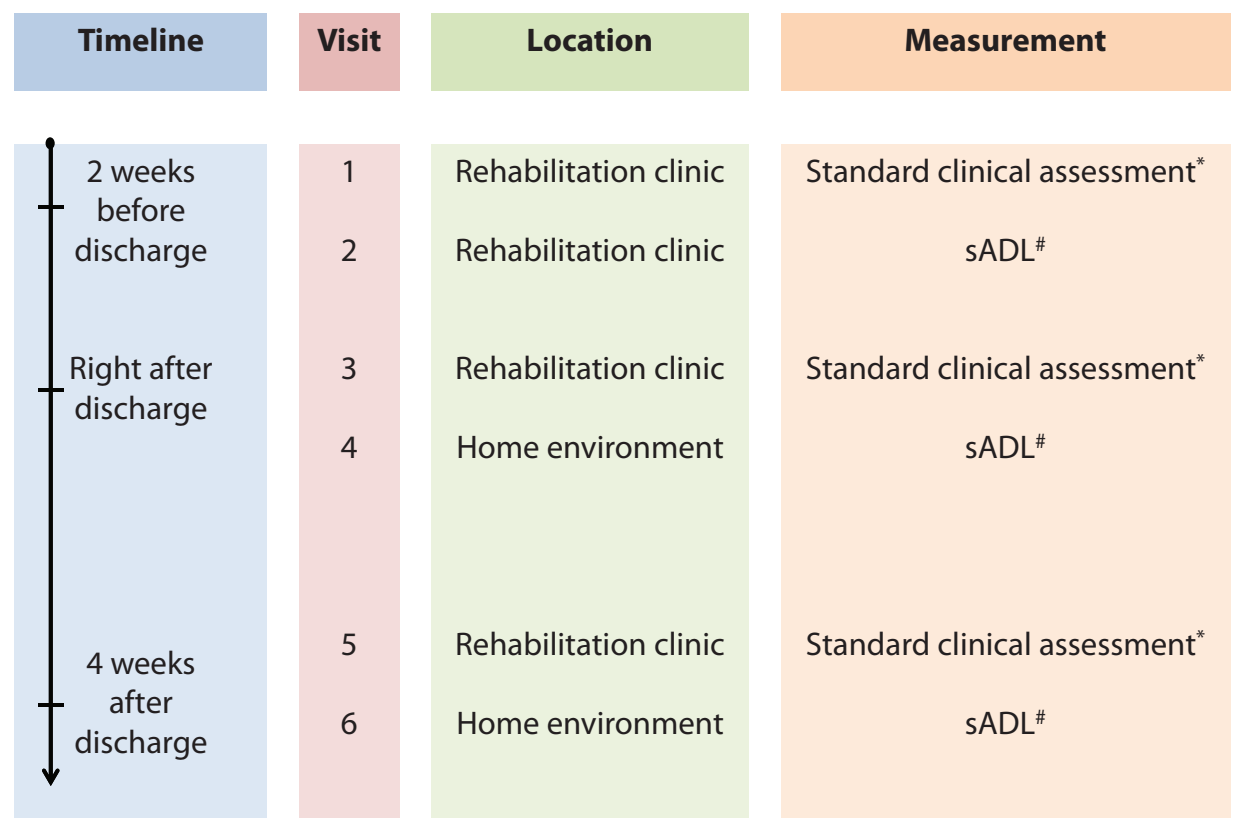

Figure 2.1: Overview of visits and assessments.

ARAT, Action Research Arm Test; FMA-UE, Fugl-Meyer Assessment - Upper Extremity; sADL, self-directed Activities of Daily Living. 
The study was approved by the Cantonal Ethics Committee Northwest and Central Switzerland (EKNZ 13101). All subjects gave written informed consent in accordance with the declaration of Helsinki.

\section{Measurement system}

Kinematic data were recorded with a Xsens full-body motion capture suit. Each IMU consists of a 3D accelerometer, a 3D magnetometer and a 3D gyroscope (Xsens Technologies, Enschede, Netherlands). To measure full-body kinematics, 14 IMUs were positioned by a therapist on the following body segments: on the instep of both feet, lower legs (medial of the tuberosity tibia), upper legs (middle part of the upper leg, on the Iliotibial tract), lower arms ( $3 \mathrm{~cm}$ distal of the wrist), upper arms ( $15 \mathrm{~cm}$ distal from the acromion), both shoulders (spine of the scapula), sternum, and the sacrum. ${ }^{22}$ Data of all sensors were captured in Xsens MVN Studio software to estimate full-body 3D kinematics, e.g., each body segment orientation, relative segment position and joint angles, ${ }^{23}$ with a sampling rate of $20 \mathrm{~Hz}$. This frequency was found to be adequate for the developed daily-life movement metrics as internal sensor data were captured at a higher frequency. ${ }^{18,22}$

Data were transferred wirelessly to a base station (Awinda Station, Xsens, the Netherlands), and connected to a laptop via USB. The base station allowed a maximal range of $10 \mathrm{~m}$ to the stroke patients. A trained research therapist monitored the system for sensor loss or system failure. To ensure good sensor quality data, the calibration procedure was performed during the measurement, if the patient changed floor level or when changes in the movement reconstructions where found indicated a sensor drift. The therapist never encouraged the patient to perform any activity. If the patient was out of range a therapist took the laptop and the base station after to the patient.

\section{Measurements}

The measurements with the full-body IMU system have been performed during the standard clinical assessment and during of self-directed Activities of Daily Living (sADL). Clinical assessments included arm motor function assessment using the FMA-UE ${ }^{3}$ and the $\mathrm{ARAT}^{4}$ to assess the patients' arm activities. In addition, the Test of Attentional Performance was included, to test the existence of a neglect. ${ }^{24}$ The assessments were performed in the clinic by a trained therapist. The SADLs were performed in the patient leisure time (clinic) and in house without any instructions. sADL data at each time point were collected for 3 hours. Measuring stroke patients' sADL that could not be possible to performed while wearing the full-body IMU system (dressing, go to the restroom, showering) were excluded from 
the daily-life measurements. Data were continuously recorded during sADL. To ensure manageable file sizes, data were saved every 10-15 minutes, after which recordings were continued without influencing the patient daily-life activities.

Measurements were performed at three time points for 3 hours (Figure 2.1: (1) 2 weeks before discharge of the rehabilitation clinic, (2) right after discharge, an (3) 4 weeks after discharge.

\section{Sensor data}

The Xsens MVN studio software (MVN Studio, Xsens, the Netherlands) was used for data capturing. Each body segment position and orientation was estimated using a Kalman filter (Xsens Kalman Filter, XKF) included in the software to generate a 3D reconstruction. ${ }^{17}$ Measurement reports, including new metrics for stroke patient evaluation, were generated in an offline environment using MATLAB ${ }^{\circledast}$ (The MathWorks Inc., Natick, MA, USA). The measurement reports use the position and orientation of each body segment to derive the joint angles. The accuracy was approximately $5 \mathrm{~mm}$ for position and $3^{\circ}$ for orientation measurements of the system for each body segment. ${ }^{25}$

Previously developed metrics for classifying activities and assessing the quality of lower and upper extremity movements were applied. ${ }^{18}$ Classification of the activities included posture detection (sitting or standing), walking detection, arm movements, and reaching detection of the affected and non-affected arm. To present large amount of aggregated sADL data in a consistent way, descriptive statistics, including average joint range of motion (RoM) (from min to max) during a reaching movement and SDs was used. ${ }^{18}$

For the upper extremities (affected and non-affected arm), the elbow and shoulder RoM, the hand position relative to the pelvis in the transversal plane, the maximum reaching distance and the reaching counts were calculated. Reaching counts were based on a hand displacement of more than $10 \mathrm{~cm}$ away from the preferred hand position (the average hand position relative to the pelvis). ${ }^{18}$ Based on this metric, the ratio of reaching counts between non-impaired and the impaired side was calculated. The reaching distance was estimated by evaluating consecutive positions of each hand expressed in the pelvis and the sternum coordinate system. ${ }^{15}$ Based on these data, the distribution of the patient's hand position in the horizontal plane was visualized. The usability of these metrics for the objective evaluation of motor performance stroke patients were found to be adequate, while a combination of metrics provided better insight in the patient sADL performance. ${ }^{26}$ 


\section{RESULTS}

\section{Subjects baseline characteristics}

Eight stroke patients (48-55 years of age) were included in this study. They had an inpatient rehabilitation stay of at least 1 month. There was a full longitudinal data set available for four of eight patients (Table 2.1). Due to technical problems related to sensor data loss and sensor drift, the other patients could not be included in the analysis.

Table 2.1: Baseline characteristics of four stroke patients

\begin{tabular}{lllll}
\hline & P1 & P2 & P3 & P4 \\
\hline Time post stroke (months) & 12 & 1 & 4 & 4 \\
Affected side & Left & Left & Right & Right \\
Dominant side & Right & Right & Right & Right \\
Neglect test (TAP\#) & None & 7 left & None & None \\
FMA-UE (total) & 57 & 55 & 57 & 7 \\
FMA-UE (proximal) & 30 & 31 & 31 & 7 \\
FMA-UE (hand/wrist) & 23 & 20 & 21 & 0 \\
FMA-UE (coordination) & 4 & 4 & 5 & 0 \\
ARAT ${ }^{\S}$ (total) & 57 & 52 & 57 & 3 \\
ARAT (grasp) & 18 & 18 & 18 & 3 \\
ARAT (grip) & 12 & 11 & 12 & 0 \\
ARAT (pinch) & 18 & 14 & 18 & 0 \\
ARAT (gross movement) & 9 & 9 & 9 & 0 \\
\hline
\end{tabular}

\#Test of Attentional Performance - Subtest Visual Field (Absence on one side).

${ }^{\dagger}$ Fugl-Meyer Assessment - Upper Extremity (0-66 points).

${ }^{\S}$ Action Research Arm Test (0-57 points).

\section{Standard clinical assessments}

Three patients (P1, P2, and P3) had mild motor upper limb impairments (FMA-UE $\geq$ 53/66 points) and one ( $\mathrm{P} 4)$ had severe motor impairment of the upper extremity (7/66 points). The arm motor function assessment (FMA-UE) improved seven points in the three patients (P1, P2, P3) with a high FMA-UE from baseline to right after discharge, but declined 4 weeks after discharge (Figure 2.2A). In the ARAT two patients (P3 and P4) improved slightly in arm activities (Figure 2.2B). One patient was diagnosed with a neglect (P2) patient, which improved over time from 7 to 4 omissions in the Test of Attentional Performance. 
A

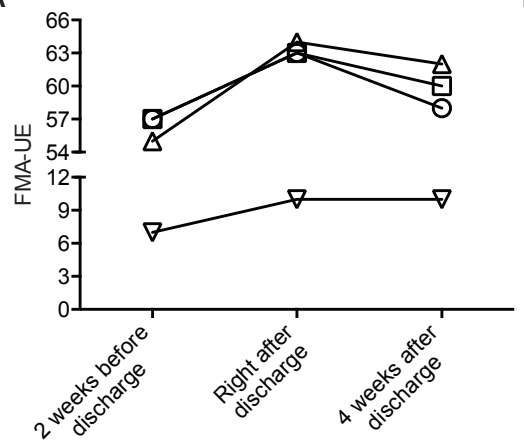

B

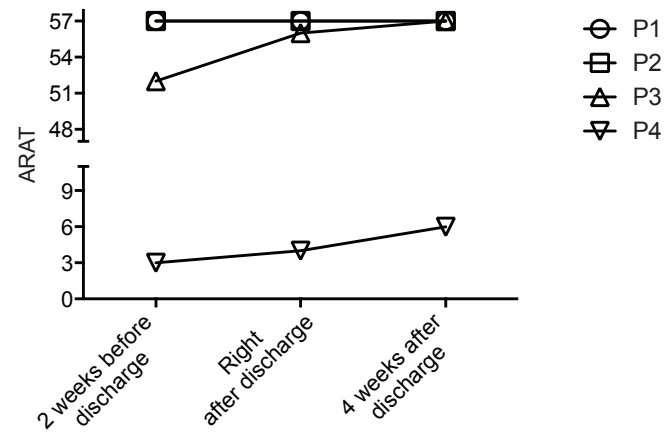

Figure 2.2: Change in clincial assessment at the three diffrent time points.

A) Fugl-Meyer Assessment - Upper Extremity (FMA-UE) - maximum 66 points. B) Action Research Arm Test (ARAT) - maximum 57 points.

\section{Continuous measurement of self-directed activities of daily living}

Table 2.2 shows the kinematic parameters collected during reaching movements: elbow flexion, shoulder abduction, and shoulder flexion (mean \pm SD over all reaching movements). The patient with the most severe motor impairments (P4) had low shoulder abduction angles at all time points and after discharge high values of elbow flexion. P2 showed improvements in all kinematic data and kept them at least partially (even further improved in shoulder flexion). The kinematic data for the other patients (P1, P3) did not show relevant over the course of rehabilitation. A change in the new metrics (reaching counts, reaching area, workspace) was seen in all subjects. Reaching counts on the impaired side from average 63 reaches (in the clinic) to 202 reaches after discharge (Figure 2.3C). Also the ratio of the reaching counts between the non-impaired and the impaired side increases 26.8\% (Figure 2.3A). Mildly affected stroke patients (P1, P2, P3) increased the

A

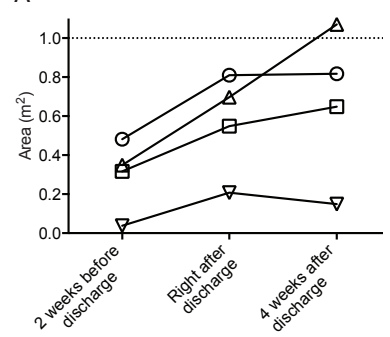

$\mathrm{B}$

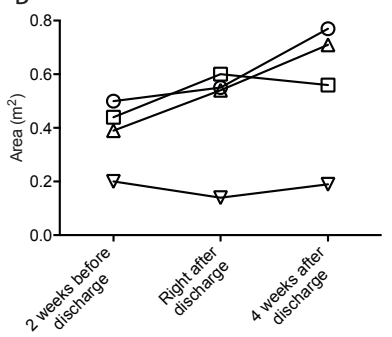

C

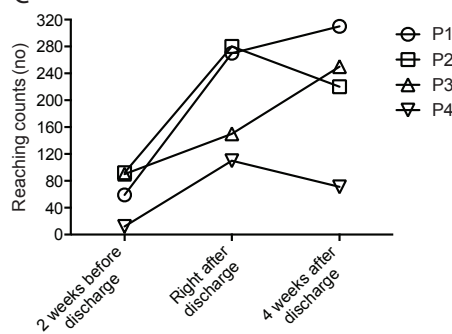

Figure 2.3: Self-directed Activities of Daily Living.

A) Ratio of reaching counts between non-impaired and the impaired side. B) Reaching area of the impaired side in the different stages of the rehabilitation. C) Reaching counts of the affected side for all patients during self-directed ADL, measured over time 3 hours. 


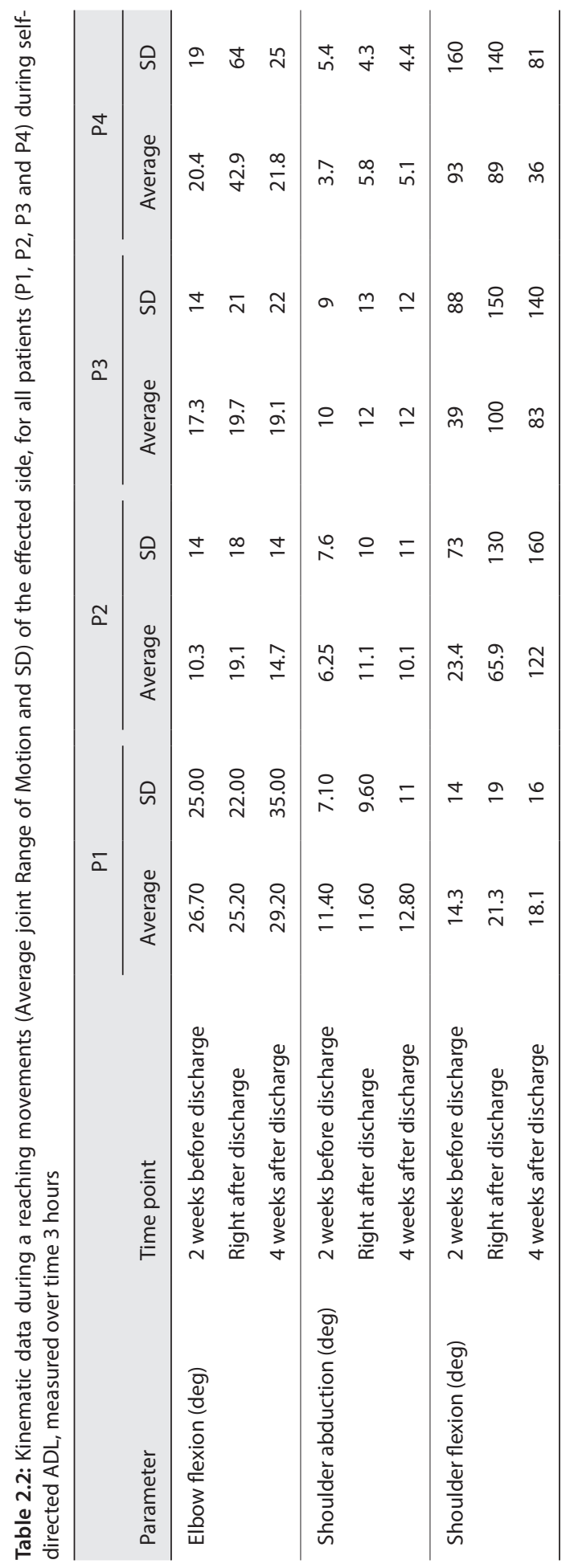


reaching area, measured during self-directed daily activities after discharge (Figures 2.3B and 2.4; Figures S2.1-S2.3 in Supplementary Material). Furthermore, P3 (right affected/ right handed) could persist the trend of increasing the reaching area $\left(0.17 \mathrm{~m}^{2}\right)$ and reaching counts (37.3\%). This is in contrast to P2 (right handed/left affected), who slightly decrease

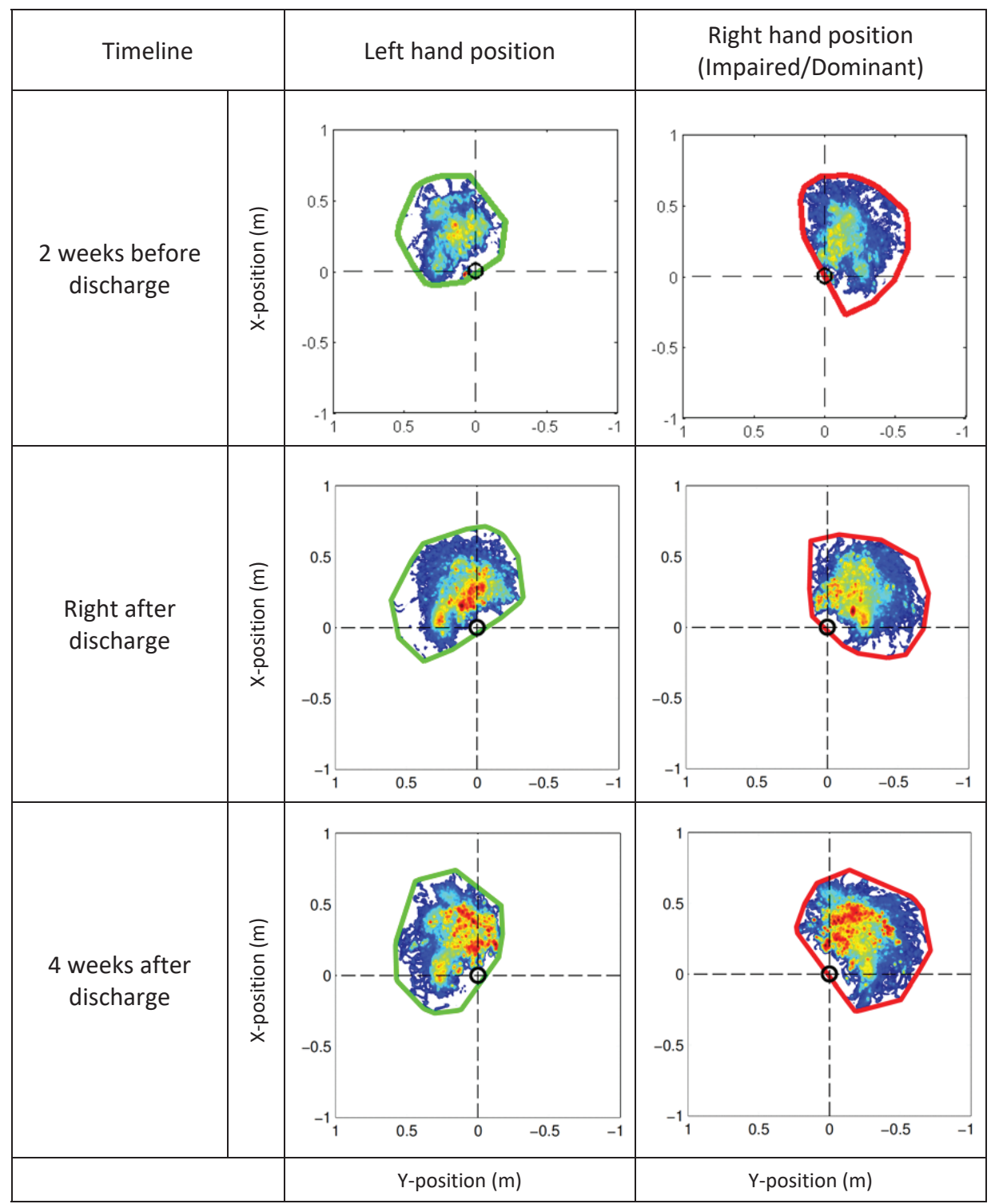

Figure 2.4: Example, of the distribution of the hand position relative to the pelvis in the horizontal plane (colours indicate the total time during the selected time slot at which the hand is in a certain position, where a darker colour reflects a longer time) of P2 at the three different stages in the rehabilitation process during self-directed Activities of Daily Living. The encircled trajectory (left hand = green, right hand $=$ red) determines the reaching area of the patient. 
his workspace after discharge $\left(0.03 \mathrm{~m}^{2}\right)$ and showed a slow increased in the reaching counts (12\%) 4 weeks after discharge. Additionally, it appears that P2 crosses the midline less with the right non-impaired hand as, compared with the impaired hand. The impaired hand is neglecting the non-impaired side (Figure 2.4).

\section{DISCUSSION}

These results demonstrate the feasibility of the method to measure upper limb kinematics, with an IMU-based motion capture system at different stages of stroke rehabilitation and during sADL and the concordance to standard clinical assessment. Although this study did not aim to compare the clinical data with the kinematic measurements, we observed a difference between the clinical assessments and the sADL measures, not only in a cross-sectional manner but also over time. The proposed metrics (reaching count, area, workspace) provide additional information as it shows an evolution, while standard clinical assessments remained stable over time after discharge. This present explorative study shows that patients with high arm function (FMA-UE) can change clinically relevant in rehabilitation. ${ }^{27}$ The data from the sADL measurements including the metrics from the sensors and the standard clinical test made it possible to characterize patients during daily-life (participation level). ${ }^{20,21}$ An understanding of the discrepancy between the clinical assessments, where the patient is encouraged by the therapist, and the patients' performance at home would help to develop tailored, innovative rehabilitation interventions, which target engagement of upper limb use in daily life. According to the current literature, this is the first study that analyzed kinematic data measured outside the clinic environment at different stages of stroke rehabilitation. While performing daily-life activities a change in arm kinematics after in-patient rehabilitation could be observed.

For the mildly impaired subjects, this was observable in the metrics reaching area, reaching counts, and ratio of reaching counts (Figure 2.3), but not in the shoulder and elbow angle ranges (Table 2.2). In the severely impaired patient, no change in the shoulder abduction angles and no change in the working area were found. This could be caused by the weakness of elbow extensors under higher shoulder load (abduction angles), which also contribute to reductions in work area. ${ }^{28}$

Previous studies using accelerometer data to calculate the ratio of impaired and nonimpaired upper limb use reported a less-symmetric and less-intense real-world bilateral upper limb activity compared with healthy subjects. ${ }^{29-31}$ Our findings are supplemented by the low amount of reaching counts on the impaired side and the difference in hand position, found in our current study that indicate a reduction of real-world upper limb 
use even in mildly effected stroke patients. Also, the differences between people living in the community and inpatient rehabilitation have not been reported in previous studies. ${ }^{29}$ Furthermore, the increase in reaching counts ratio between the impaired and non-impaired arm after rehabilitation in all patients would also suggest that patients have to be motivated to use their hands more in the leisure time during the inpatient stay.

When looking at the single arm use (Figure 2.4), the new developed metric (work area) offers the possibility to assess and plan interventions for motor neglect. These results supports the findings from Ogourtsova et al. ${ }^{32}$ that neglect contribute to deficits observed in action execution of the non-affected limb.

\section{Limitation}

To measure stroke patients, sADLs are challenging but promising. The main limitation of this feasibility study is the low number of stroke patients included. From eight post-stroke patients who where equipped with the full-body motion capture system, data from only four patients were suitable for analysis. The data from the four excluded patients were not usable due to sensor orientation (sensor drift and sensor placement) and transmitting problems from sensors to the receiving device. The importance of the sensor calibration procedures, the influence of the environmental factors (e.g., change in floor levels, electronic devices in home), the duration of measurements, and the complexity of activities of the patients affected the measurements. ${ }^{33}$ This could be solved with more robust sensing and communication systems in the future. It is unclear what patients did during the 3 hours of sADL, as tasks could highly influence upper limb kinematics.

A combination of sensors and a more extensive activity monitoring system including a markerless camera system could increase the knowledge about the patient performance. ${ }^{34}$ Also the obtrusive measurement setup (14 sensors) makes it less suitable for long-term measurements, without technical support in stroke subjects. Furthermore, the presence of the therapist could influence the patient performance during the measurement. A reduced sensor set would improve the problem of obtrusiveness. ${ }^{13,35}$

Moreover, a group analysis was not possible because of data loss of four subjects and the heterogeneity of the stroke population. 


\section{CONCLUSIONS}

This study showed the feasibility of measuring kinematics in stroke patients at the different stages of rehabilitation. Our results illustrate that certain metrics derived from kinematic data are likely more sensitive to changes as compared with clinical assessments. Measuring with a full-body IMU system allows a quantification of movement quality outside a laboratory environment. Future studies are needed to optimize the technology, better characterize the metrics derived from IMUs, and include more post-stroke patients to profile the rehabilitation process.

\section{Acknowledgements}

The authors would like to thank Fokke van Meulen and Marcel Weusthof for their help in the implementation of the monitoring system, Irene Christen and Lydia Fischer for their support with the study, and Janne M. Veerbeek for their valuable help in the data analysis, as well as all patients who participated in the study.

\section{REFERENCES}

1. Murray CJ, Vos T, Lozano R, Naghavi M, Flaxman AD, Michaud C, Ezzati M, et al. Disabilityadjusted life years (DALYs) for 291 diseases and injuries in 21 regions, 1990-2010: a systematic analysis for the Global Burden of Disease Study 2010. Lancet. 2012;380(9859):2197-2223.

2. Kwakkel G, Kollen BJ, van der Grond J, Prevo AJ. Probability of regaining dexterity in the flaccid upper limb: impact of severity of paresis and time since onset in acute stroke. Stroke. 2003;34(9):2181-2186.

3. Fugl-Meyer AR, Jaasko L, Leyman I, Olsson S, Steglind S. The post-stroke hemiplegic patient. 1. a method for evaluation of physical performance. Scandinavian Journal of Rehabilitation Medicine. 1975;7(1):13-31.

4. Lyle RC. A Performance-Test for Assessment of Upper Limb Function in Physical Rehabilitation Treatment and Research. International Journal of Rehabilitation Research. 1981;4(4):483-492.

5. Alt Murphy M, Willen C, Sunnerhagen KS. Responsiveness of upper extremity kinematic measures and clinical improvement during the first three months after stroke. Neurorehabil Neural Repair. 2013;27(9):844-853.

6. Alt Murphy M, Willen C, Sunnerhagen KS. Kinematic variables quantifying upper-extremity performance after stroke during reaching and drinking from a glass. Neurorehabil Neural Repair. 2011;25(1):71-80.

7. Cirstea MC, Levin MF. Compensatory strategies for reaching in stroke. Brain. 2000;123 ( Pt 5):940-953.

8. Levin MF. Interjoint coordination during pointing movements is disrupted in spastic hemiparesis. Brain. 1996;119 ( Pt 1):281-293.

9. Subramanian SK, Levin MF. Viewing medium affects arm motor performance in 3D virtual environments. Journal of NeuroEngineering and Rehabilitation. 2011;8:36. 
10. de los Reyes-Guzman A, Dimbwadyo-Terrer I, Trincado-Alonso F, Monasterio-Huelin F, Torricelli D, Gil-Agudo A. Quantitative assessment based on kinematic measures of functional impairments during upper extremity movements: A review. Clinical biomechanics (Bristol, Avon). 2014;29(7):719-727.

11. Stewart JC, Cramer SC. Patient-reported measures provide unique insights into motor function after stroke. Stroke. 2013;44(4):1111-1116.

12. Uswatte G, Foo WL, Olmstead H, Lopez K, Holand A, Simms LB. Ambulatory monitoring of arm movement using accelerometry: An objective measure of upper-extremity rehabilitation in persons with chronic stroke. Archives of Physical Medicine and Rehabilitation. 2005;86(7):14981501.

13. Leuenberger K, Gonzenbach R, Wachter S, Luft A, Gassert R. A method to qualitatively assess arm use in stroke survivors in the home environment. Medical \& Biological Engineering \& Computing. 2016:1-10.

14. Patel S, Park H, Bonato P, Chan L, Rodgers M. A review of wearable sensors and systems with application in rehabilitation. Journal of Neuroengineering and Rehabilitation. 2012;9.

15. Steins D, Dawes H, Esser P, Collett J. Wearable accelerometry-based technology capable of assessing functional activities in neurological populations in community settings: a systematic review. Journal of NeuroEngineering and Rehabilitation. 2014;11:36.

16. van Meulen FB, Reenalda J, Buurke JH, Veltink PH. Assessment of daily-life reaching performance after stroke. Annals of Biomedical Engineering. 2015;43(2):478-486.

17. Roetenberg D, Luinge H, Slycke P. Xsens MVN: full 6DOF human motion tracking using miniature inertial sensors. Xsens Motion Technologies BV, Tech. Rep. 2009.

18. van Meulen FB, Klaassen B, Held J, Reenalda J, Buurke JH, van Beijnum B-JF, Luft A, et al. Objective evaluation of the quality of movement in daily life after stroke. Frontiers in Bioengineering and Biotechnology. 2016;3(210).

19. Schweighofer N, Han CE, Wolf SL, Arbib MA, Winstein CJ. A functional threshold for longterm use of hand and arm function can be determined: Predictions from a computational model and supporting data from the extremity constraint-induced therapy evaluation (EXCITE) trial. Physical Therapy. 2009;89(12):1327-1336.

20. Uswatte G, Miltner WHR, Foo B, Varma M, Moran S, Taub E. Objective measurement of functional upper-extremity movement using accelerometer recordings transformed with a threshold filter. Stroke. 2000;31(3):662-667.

21. Andre JM, Didier JP, Paysant J. "Functional motor amnesia" in stroke (1904) and "learned non-use phenomenon" (1966). Journal of Rehabilitation Medicine. 2004;36(3):138-140.

22. Klaassen B, van Beijnum BJ, Weusthof M, Hofs D, van Meulen F, Droog E, Luinge H, et al. A Full Body Sensing System for Monitoring Stroke Patients in a Home Environment. Biomedical Engineering Systems and Technologies, Biostec 2014. 2015;511:378-393.

23. Roetenberg D, Luinge HJ, Baten CT, Veltink PH. Compensation of magnetic disturbances improves inertial and magnetic sensing of human body segment orientation. IEEE Transactions on Neural Systems and Rehabilitation Engineering. 2005;13(3):395-405.

24. Zimmermann P, Fimm B. A test battery for attentional performance. Applied neuropsychology of attention. Theory, diagnosis and rehabilitation. 2002:110-151.

25. Roetenberg D, Baten CT, Veltink PH. Estimating body segment orientation by applying inertial and magnetic sensing near ferromagnetic materials. IEEE Transactions on Neural Systems and Rehabilitation Engineering. 2007;15(3):469-471.

26. Klaassen B, van Beijnum BF, Held JP, Reenalda J, van Meulen FB, Veltink PH, Hermens HJ. Usability evaluations of a wearable Inertial sensing system and quality of movement metrics for stroke survivors by care professionals. Frontiers in Bioengineering and Biotechnology. 2017; 5:20. 
27. Page SJ, Fulk GD, Boyne P. Clinically important differences for the upper-extremity FuglMeyer Scale in people with minimal to moderate impairment due to chronic stroke. Physical Therapy. 2012;92(6):791-798.

28. Sukal TM, Ellis MD, Dewald JP. Shoulder abduction-induced reductions in reaching work area following hemiparetic stroke: neuroscientific implications. Experimental Brain Research. 2007;183(2):215-223.

29. Bailey RR, Klaesner JW, Lang CE. Quantifying real-world upper-limb activity in nondisabled adults and adults with chronic stroke. Neurorehabil Neural Repair. 2015;29(10):969-978.

30. van der Pas SC, Verbunt JA, Breukelaar DE, van Woerden R, Seelen HA. Assessment of arm activity using triaxial accelerometry in patients with a stroke. Archives of Physical Medicine and Rehabilitation. 2011;92(9):1437-1442.

31. Michielsen ME, Selles RW, Stam HJ, Ribbers GM, Bussmann JB. Quantifying nonuse in chronic stroke patients: a study into paretic, nonparetic, and bimanual upper-limb use in daily life. Archives of Physical Medicine and Rehabilitation. 2012;93(11):1975-1981.

32. Ogourtsova T, Archambault P, Lamontagne A. Impact of post-stroke unilateral spatial neglect on goal-directed arm movements: systematic literature review. Topics in Stroke Rehabilitation. 2015;22(6):397-428.

33. Robert-Lachaine X, Mecheri H, Larue C, Plamondon A. Validation of inertial measurement units with an optoelectronic system for whole-body motion analysis. Medical \& Biological Engineering \& Computing. 2017;55(4):609-619.

34. Sevrin L, Noury N, Abouchi N, Jumel F, Massot B, Saraydaryan J. Detection of collaborative activity with Kinect depth cameras. Paper presented at: Engineering in Medicine and Biology Society (EMBC), 2016 IEEE 38th Annual International Conference of the2016.

35. Van Meulen FB, van Beijnum B-JF, Buurke JH, Veltink PH. Assessment of lower arm movements using one inertial sensor. Paper presented at: Rehabilitation Robotics (ICORR), 2017 International Conference on 2017. 


\section{SUPPLEMENTARY MATERIAL}

The Supplementary Material for this article can be found online at https://www.frontiersin. org/articles/10.3389/fbioe.2018.00027/ full\#supplementary-material.

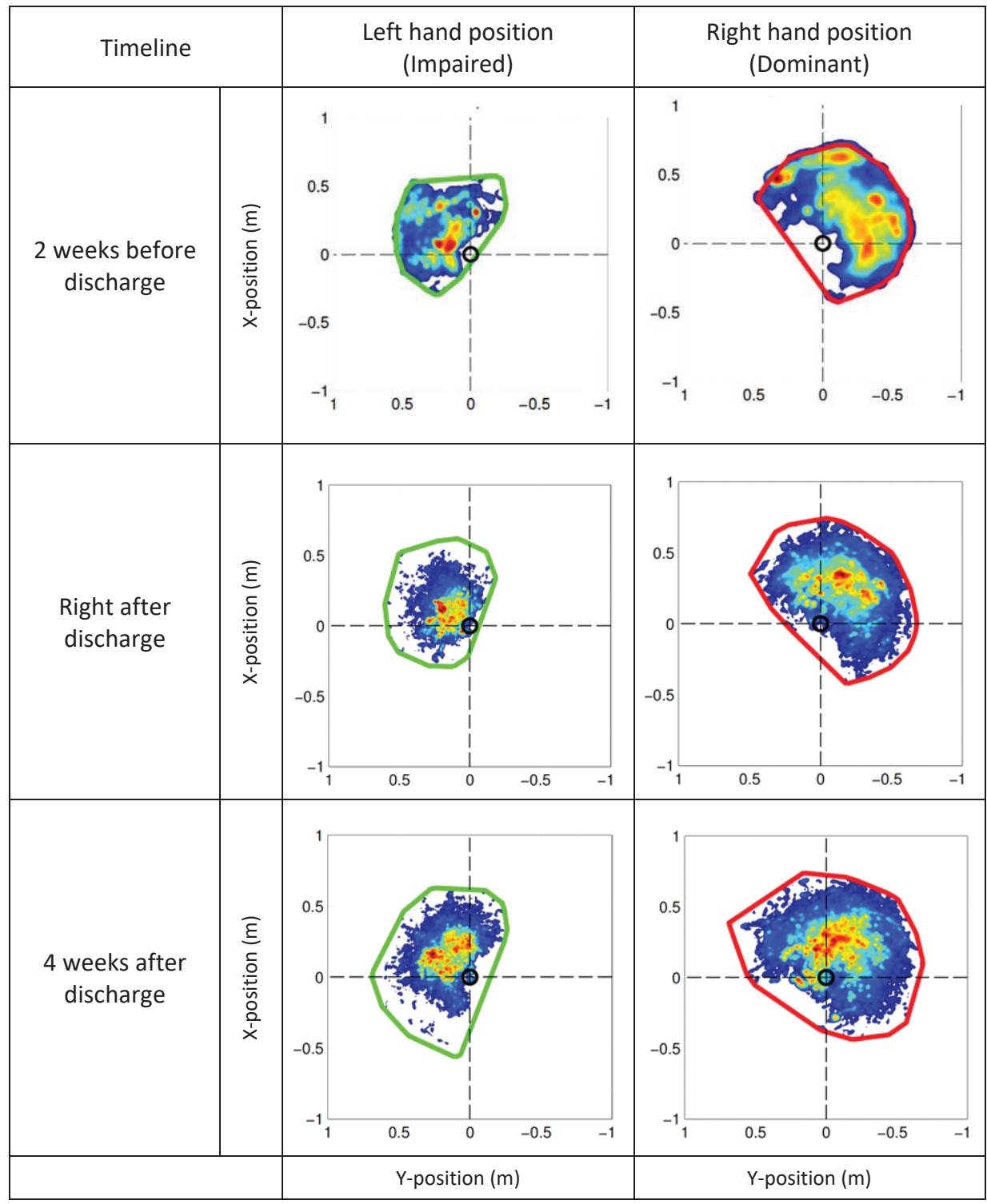

Supplementary Figure S2.1: The distribution of the hand position relative to the pelvis (colours indicate the total time during the selected time slot at which the hand is in a certain position: dark red = mostfrequent position, blue $=$ least-frequent position) of $\mathrm{P} 1$ at the three different stages in the rehabilitation process during self-directed Activities of Daily Living.

The encircled trajectory (left hand = green, right hand $=$ red) determine the reaching area of the patient. 


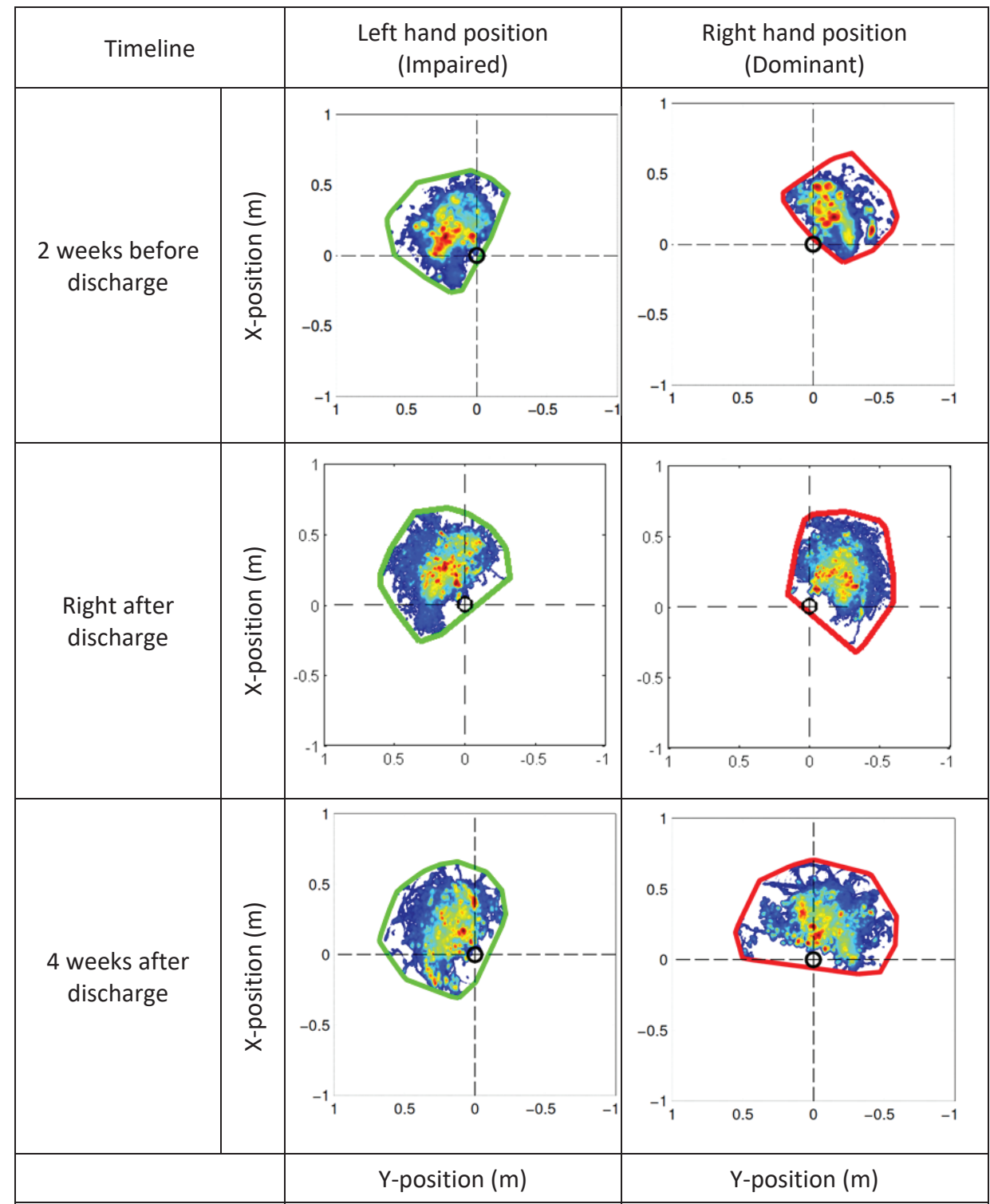

Supplementary Figure S2.2: The distribution of the hand position relative to the pelvis (colours indicate the total time during the selected time slot at which the hand is in a certain position: dark red = mostfrequent position, blue $=$ least-frequent position) of P3 at the three different stages in the rehabilitation process during self-directed Activities of Daily Living.

The encircled trajectory (left hand = green, right hand = red) determine the reaching area of the patient. 


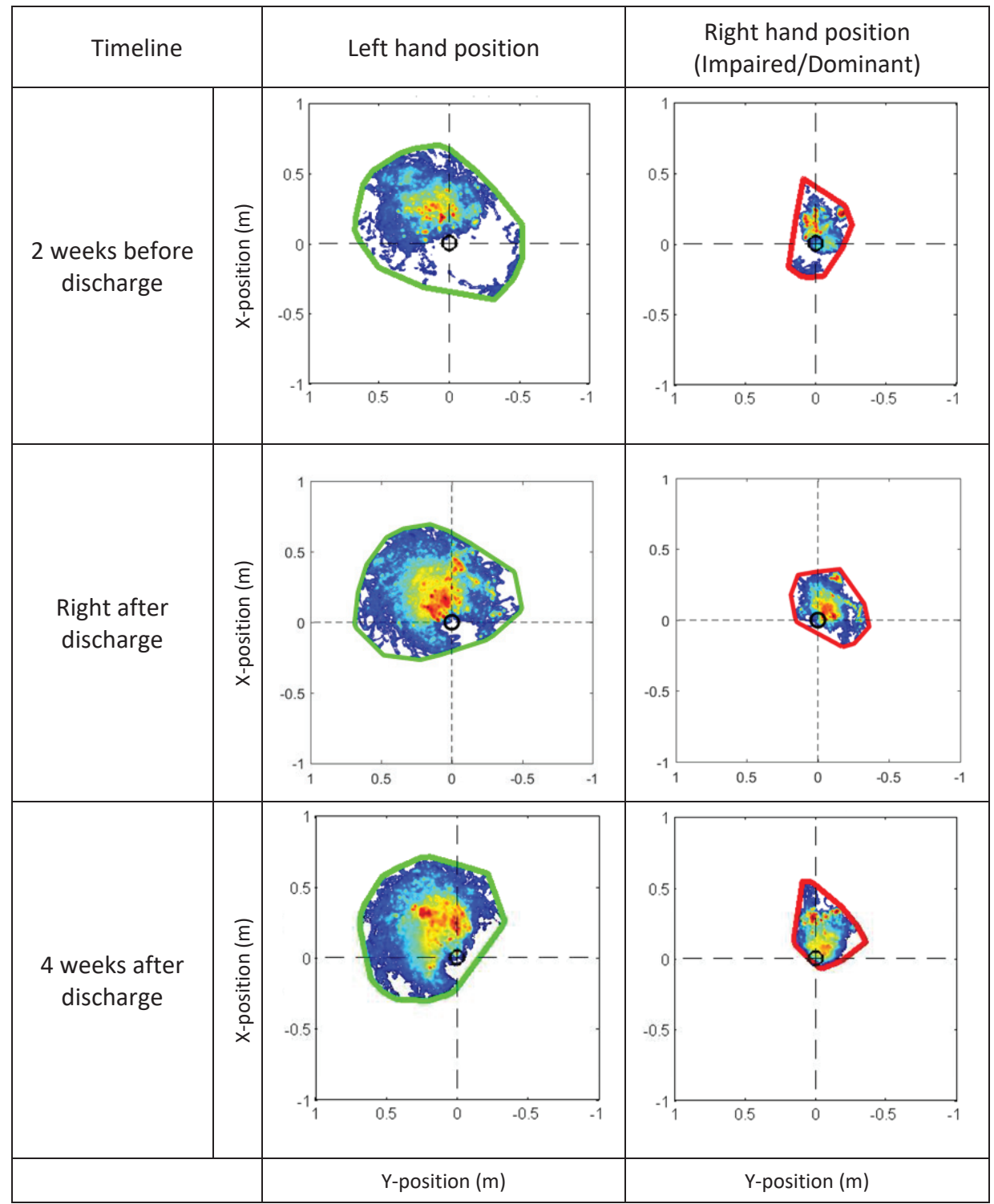

Supplementary Figure S2.3: The distribution of the hand position relative to the pelvis (colours indicate the total time during the selected time slot at which the hand is in a certain position: dark red = mostfrequent position, blue $=$ least-frequent position) of $\mathrm{P} 4$ at the three different stages in the rehabilitation process during self-directed Activities of Daily Living.

The encircled trajectory (left hand = green, right hand = red) determine the reaching area of the patient. 



\section{Chapter 3}

\section{Usability evaluation of a vibrotactile feedback system in stroke subjects}

J.P.O. Held*, B. Klaassen, B-J.F. van Beijnum, A.R. Luft, P.H. Veltink

* Both authors contributed equally. 


\section{ABSTRACT}

Background To increase the functional capabilities of stroke subjects during activities of daily living, patients receive rehabilitative training to recover adequate motor control. With the goal to motivate self-training by use of the arm in daily life tasks, a sensor system (Arm Usage Coach, AUC) was developed that provides VibroTactile (VT) feedback if the patient does not move the affected arm above a certain threshold level. The objective of this study is to investigate the usability of this system in stroke subjects.

Method The study was designed as a usability and user acceptance study of feedback modalities. Stroke subjects with mild to moderate arm impairments were enrolled. The subjects wore two AUC devices one on each wrist. VT feedback was given by the device on the affected arm. A semi-structured interview was performed before and after a measurement session with the AUC. In addition, the System Usability Scale (SUS) questionnaire was given.

Results Ten ischemic chronic stroke patients ( $39 \pm 38$ months after stroke) were recruited. Four out of ten subjects have worn the VT feedback on their dominant, affected arm. In the pre-measurement interview, eight participants indicated a preference for acoustic or visual over VT feedback. In the post evaluation interview, nine of ten participants preferred VT over visual and acoustic feedback. On average, the AUC gave VT feedback six times during the measurement session. All participants, with the exception of one, used their dominant arm more than the non-dominant. For the SUS, eight participants responded above 80, one between 70 and $80 \%$, and one participant responded below $50 \%$.

Discussion More patients accepted and valued VT feedback after the test period, hence VT is a feasible feedback modality. The AUC can be used as a telerehabilitation device to train and maintain upper extremity use in daily-life tasks. 


\section{INTRODUCTION}

To gain independence and increase the quality of life, inpatient neurorehabilitation is usually necessary for hemiparetic stroke subjects. ${ }^{1}$ The functional capabilities of these patients are assessed using standardized tests, which are intended to predict functional performance after discharge. However, the power of this prediction is poor. ${ }^{2}$ Therefore, daily-life monitoring of movement quality and quantity would help in guidance of therapy. We previously developed a monitoring solution using a full body inertial sensor suit, ${ }^{3,4}$ with resulting metrics capable of objectifying the quality of movement of stroke subjects. Monitoring in poststroke patients demonstrated that while patients are capable of performing movements during the clinical assessments, they often do not use their affected arm in daily life. ${ }^{5}$ These results suggest that capability and arm training does not automatically translate into usage of the affected arm. An unobtrusive coaching system for arm usage during daily life might be able to motivate arm movement in these patients.

In addition to the INTERACTION project, a reduced sensor system was developed with the objective to coach and motivate stroke subjects in remembering to use their affected arm during daily life activities. This Arm Usage Coach (AUC) includes two inertial sensors and one VibroTactile (VT) device. The objective here is to investigate if VT feedback is accepted and the usability of the AUC in stroke subjects during simulated daily life activities. The development of the first prototype and the evaluation with healthy subjects is described in Klaassen et al. ${ }^{6}$ This paper is a usability study of the first prototype with stroke patients.

\section{METHODS AND MATERIALS}

\section{Study overview}

This study was designed as a usability study, conducted at the University Hospital Zurich, to investigate the usability and the acceptance of the AUC. Stroke subjects with mild to moderate arm impairments were enrolled. A semi-structured interview was performed at enrollment, including a questionnaire, to assess the preference of different types of feedback modalities, e.g., VT, visual, and acoustic feedback among stroke subjects. Then, a measurement session was performed using the AUC to let subjects experience VT feedback, responsive to their arm activity and the overall usage of the device. Afterward another semi-structured interview was done, and the System Usability Scale (SUS) ${ }^{7}$ questionnaire was applied to evaluate the system's usability. An overview is shown in Figure 3.1. 


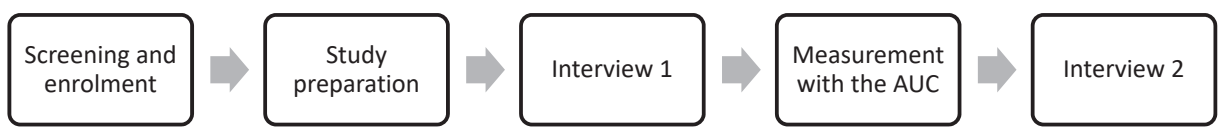

Figure 3.1: Flowchart of the study.

\section{Participant selection}

Stroke subjects (above 18 years old) with a unilateral ischemic or hemorrhagic stroke and residual hemiparesis after completion of inpatient rehabilitation were enrolled between March and April 2016. Stroke subjects were required to have a mild to moderate arm impairment with a Fugl-Meyer Assessment - Upper Extremity (FMA-UE, score range 0-66) score higher than $22 .{ }^{8}$ Additional exclusion criteria were as follows: if the participant has: (1) a major untreated depression, (2) a major cognitive or communication deficits, (3) a major comprehension or memory deficits, (4) major medical comorbidity, (5) severely impaired sensation, (6) sever neglect, and (7) suffering from comprehensive aphasia. Furthermore, the aim for this usability study is to include 10 participants.

\section{Preparation of the study}

The participants gave written informed consent in accordance with the declaration of Helsinki. The Cantonal ethics in Zurich gave approval in using the VT feedback system (nr. 06-2016). Demographic data of the participant (including age, gender, stroke event, work status, technical background, left or right handed, affected side, and arm dimensions) were documented. Furthermore, vibration sense on the affected arm was assessed using the Revised Nottingham sensory assessment (on the wrist). ${ }^{9}$

\section{Preinterview}

A semi-structured interview was performed with each participant before the measurement intervention. The questions, with multiple answering options, are listed in Table 3.1.

\section{Arm Usage Coach overview}

The AUC is composed of two inertial sensors (Xsens B.V.1) (each weights $27 \mathrm{~g}$ ), an Elitac (Elitac B.V.2) VT actuator (weighting $200 \mathrm{~g}$ ), and a laptop. ${ }^{4}$ Both sensors are wirelessly connected via an Xsens dongle, utilizing the Awinda protocol, and the Elitac system via Bluetooth. The inertial sensors are worn on each wrist of the participant. The Elitac VT actuator is placed, with Velcro on the affected arm of the participant (Figure 3.2). The 
Table 3.1: Questions during pre-interview

\begin{tabular}{|c|c|c|}
\hline$\#$ & Question & Answering options \\
\hline 1 & Do you use a self-tracking device? & $\begin{array}{l}\text { Yes/No. If yes, what type? Smartphone, wrist } \\
\text { band, walking tracker, sleeping mat, other.... }\end{array}$ \\
\hline 2 & $\begin{array}{l}\text { Do you have any experience with getting } \\
\text { feedback? }\end{array}$ & $\begin{array}{l}\text { Yes/No. If yes, by whom? Therapist, doctor, } \\
\text { relatives, friends, other...) }\end{array}$ \\
\hline 3 & Do you get therapy for the upper extremities? & Yes/No \\
\hline 4 & What kind of feedback do you prefer? & Visual, acoustic, vibrotactile, none \\
\hline 5 & When should the feedback be applied? & $\begin{array}{l}\text { Every } 15 \mathrm{~min} \text {, per hour, every second hour, } \\
\text { if the arm is not moving, one time per day, } \\
\text { none... }\end{array}$ \\
\hline 6 & $\begin{array}{l}\text { Should the information about the feedback be } \\
\text { send to the clinician? }\end{array}$ & Yes/No \\
\hline
\end{tabular}

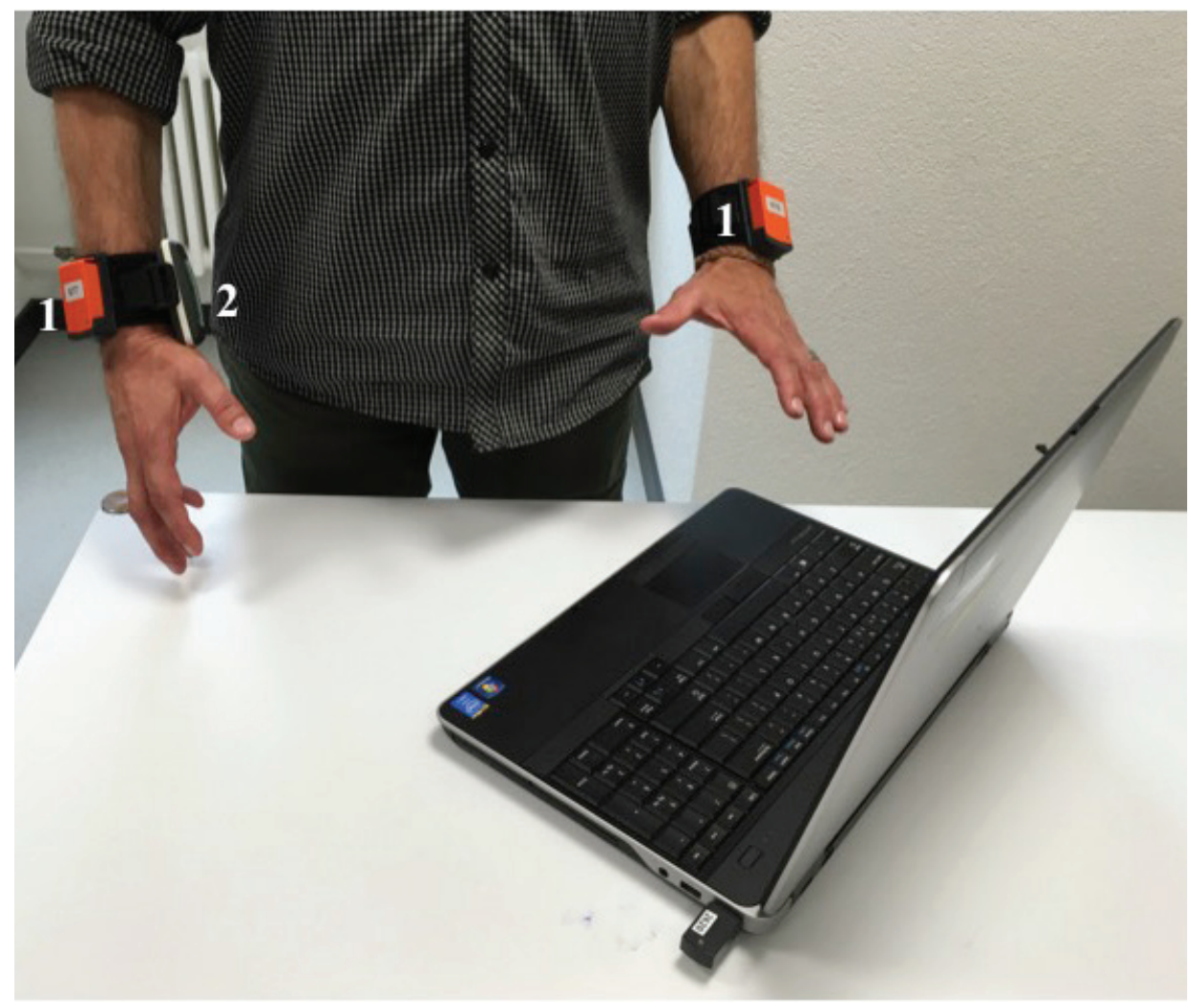

Figure 3.2: Right impaired stroke subject with Arm Usage Coach prototype. 1) Inertial sensors; 2) Elitac VT actuator. 
laptop is operating a software program for providing feedback, including analysis of the sensor data, a decision feature, and feedback.

A mandatory starting pose is required from the participant, which is used as a reference pose to compute arm activity by using a metric called the difference acceleration vector (DAV). ${ }^{4}$ The length of the DAV $d(t)$ is calculated by subtracting a reference gravitational acceleration vector $g(t)$, obtained from the sensor data captured during the reference pose, from the current acceleration vector $a(t)$ of the sensor during daily life movements, and taking the norm of the resulting vector.

$$
d(t)=\sqrt{\left(a_{x}(t)-g_{x, t 0}\right)^{2}+\left(a_{y}(t)-g_{y, t 0}\right)^{2}+\left(a_{z}(t)-g_{z, t 0}\right)^{2}}
$$

Finally, the mean DAV is calculated over a 1-s time period of measurement data. The final decision of determining whether a certain mean DAV can be seen as arm movement activity is based on more complex algorithms, as explained in Klaassen et al., 2016. ${ }^{4}$ These decision-making algorithms can be personalized by the following two input parameters of the software, namely, (1) threshold of arm activities (between 0 and 9) and (2) the ratio between the affected and non-affected arm usage $(0$ - 1 , where 1 means the affected side should be used in the same amount as the non-affected side). The outcome parameters of the algorithms are amount of arm usage (when exceeding the threshold mentioned above, for the left and right arm as percentage of combined arm usage) and the amount of feedback provided over time. A default set of input parameters is used for the software for each participant (threshold $=8 \mathrm{~m} / \mathrm{s}^{2}$ and ratio $=1$ ). The VT feedback is given at 158.3 $\pm 2.4 \mathrm{~Hz}$ and is given for only $489 \mathrm{~ms}$ (300 ms duration, and 189 spin-up and down time of the vibration motor to reach the vibration intensity).

\section{Measurement protocol}

At the start of the measurement, participants were asked to don the wristbands, which include inertial sensor holders, then click the sensors into the holder and finally mount the VT actuator on the Velcro-wristband on the affected side. Then, the participants were instructed to stand in a comfortable neutral position. This will be the reference position in which arm activity is detected. ${ }^{4}$

Next, a selection of tasks, listed in Table 3.2, is performed by the participant twice in a specific measurement area. This measurement area consists of one room $\left(18 \mathrm{~m}^{2}\right)$ including a table and a chair, with a door leading to a $15 \mathrm{~m}$ long hallway. This set of tasks is performed twice, one time where the VT feedback device is OFF and a second time where the device is turned $\mathrm{ON}$ for later comparison. 
Table 3.2: Activity protocol

\begin{tabular}{ll}
\hline$\#$ & Tasks \\
\hline 1. & Sit in a chair behind a table in the ARAT test room \\
2. & Stand up and walk to the door \\
3. & Open the door, walk through it to the hallway and close it again \\
4. & Walk 15 meters in the hallway. \\
5. & Turn around \\
6. & Walk 15 meters \\
7. & Open the door, walk through it to the ARAT test room and close it again \\
8. & Walk to the table \\
9. & Move objects from A to B according to the ARAT assessment test in standing \\
10. & Take a seat in a chair \\
\hline
\end{tabular}

Task 1, 2, 3, 7, 8 and 10 are based on the protocol presented by van Meulen et al., 2015. ${ }^{10}$ The tasks were specifically designed for measuring stroke subjects performing simulated activities of daily living. Participants were instructed to stand up from the chair, walk to a door, open the door, walk through the door and close the door. Then, the participants were instructed to walk in the hallway for $15 \mathrm{~m}$, turn around and walk $15 \mathrm{~m}$ back to the door, open, walk through the door, close the door and walk to a table. On this table (height 75 $\mathrm{cm}$ ), four blocks $\left(10,2.5,5,7.5 \mathrm{~cm}^{3}\right)$, a cricket ball, a sharpening stone, a drinking glass, and a marble were placed. Participants were asked to grasp each object and place them on a shelf. This combined set is part of the ARAT ${ }^{11}$ assessment while standing. After all, items were placed on the shelf, and the participants were instructed to sit down in a chair. After the measurement, arm usage and the amount of feedback that is given were presented in a visual graph on a computer screen, as shown in Figure 3.3.

A Percentage of time of arm usage

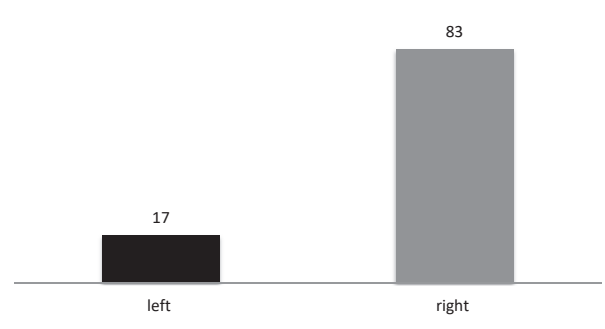

B VT feedback given to subject

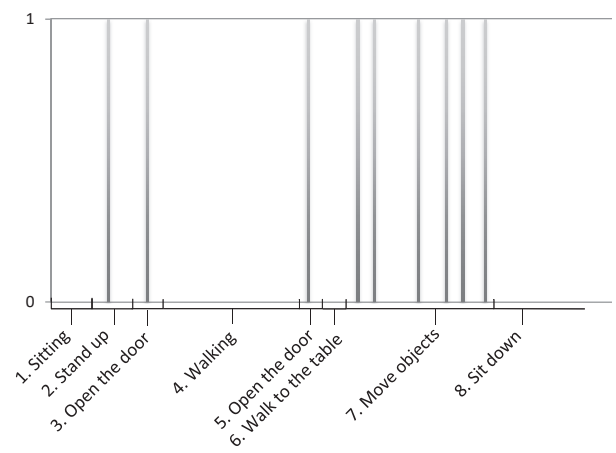

Figure 3.3: Example of arm usage and VibroTactile (VT) feedback results. A) Percentage of time of arm usage and B) VT feedback over time. 


\section{Postinterview}

A semi-structured interview was done after the measurements. Two questionnaires were presented to the participants: (1) a custom-made questionnaire as listed in Table 3.3 and (2) the SUS. ${ }^{7}$ The SUS is a well-established 10-item scale, designed to evaluate usability (effectiveness, efficiency, and satisfaction) of technical devices. Questions were scored on a five- point Likert-type scale ranging from "strongly agree" to "strongly disagree." Combined scores were translated to a range of $0-100$, with a higher score meaning better usability. ${ }^{7}$ SUS scores above $90 \mathrm{~s}$ reflect best imaginable usability, 85 excellent, 71 good, and 50 suggest fair usability. Scores below 50 indicate that using the product or intervention in practice with will be limited due to low compliance. ${ }^{12,13}$ An additional customized questionnaire (Table 3.4) was designed to gain more insight into the patient's preferences in terms of feedback after using the AUC and if they would like to use the AUC at home to increase arm function in daily life.

Table 3.3: Custom questionnaire during the postinterview

\begin{tabular}{lll}
\hline$\#$ & Question & Answering options \\
\hline 1 & What kind of feedback would you prefer? & Visual, acoustic, vibrotactile, none \\
2 & When should the feedback be applied? & $\begin{array}{l}\text { Every } 15 \text { min, per hour, every second hour, if the } \\
\text { arm is not moving, one time per day, none... }\end{array}$ \\
3 & $\begin{array}{l}\text { Should the information about the feedback be } \\
\text { send to the clinician? }\end{array}$ & Yes/No \\
4 & $\begin{array}{l}\text { Would you use a device like the AUC? } \\
5\end{array}$ & When would you use the AUC? \\
& $\begin{array}{l}\text { Do you think the AUC could compliment your } \\
\text { standard therapy? }\end{array}$ & Yes/No \\
\end{tabular}

\section{RESULTS}

\section{Participant enrolment}

Ten subjects of an ischemic stroke (39 \pm 38 months after the event) were recruited in the University Hospital Zurich. Four out of 10 participants wore the AUC on the dominant, impaired arm. Six participants had arm FMA-UE score of larger or equal to 48 points; four participants showed poor to limited arm function (FMA-UE $\leq 47$ points). Details of each participant are listed in Table 3.4. Eight participants had impairments in vibration sense $(>64 \mathrm{~Hz}$ ) on the wrist, at the radial and ulnar styloid process and between the processes. Six participants reported to have a technical occupational background. Seven participants 


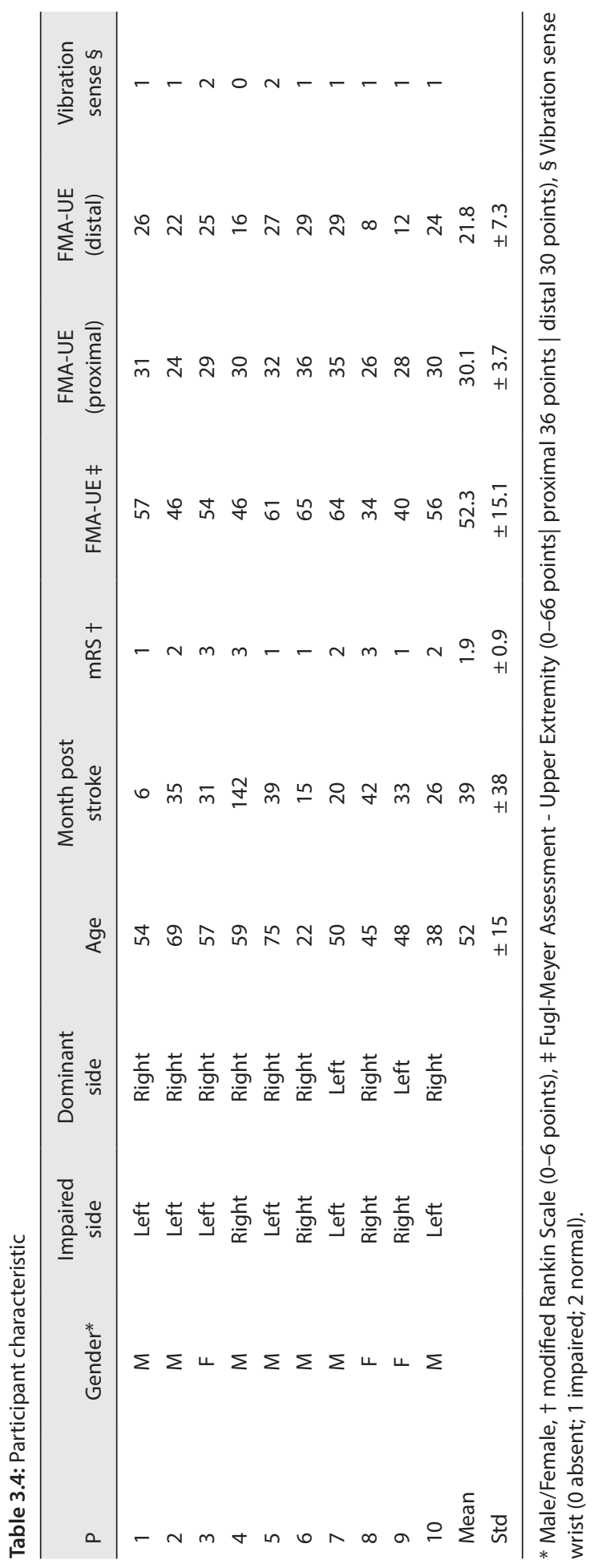


have used self-tracking devices before, for example, a pulse watch and walk-ing trackers. One participant used an activity tracker worn on the wrist to monitor his arm movements during daily life.

\section{Preinterview results}

The results from the questionnaire given during the preinterview are listed in Table 3.5. Seven participants had experience with self-tracking devices, e.g., wrist band, walking trackers, or chest strap to measure heart rate. Seven participants mentioned that they have experience with feedback on arm movement provided by relatives, friends, therapists, or self-tracking devices. Eight participants preferred acoustic or visual over VT feedback based on their experience. Four participants mentioned that they would like to receive feedback hourly or when the arm is not moving, one participant every $15 \mathrm{~min}$, and one patient once daily. All participants agreed on sharing the feedback information with a clinician.

Table 3.5: Results interview 1

\begin{tabular}{|c|c|c|}
\hline \# & Question & Results \\
\hline 1 & Do you use a self-tracking device? & Yes: 7; No: 3 \\
\hline 2 & $\begin{array}{l}\text { Do you have any experience with getting } \\
\text { feedback? }\end{array}$ & Yes: 7; No: 3 \\
\hline 3 & Do you get therapy for the upper extremities? & Yes: 5 ; No: 5 \\
\hline 4 & What kind of feedback would you prefer? & Visual: 2 ; acoustic: 6 ; vibrotactile: 3 ; none: 0 \\
\hline 5 & When should the feedback be applied? & $\begin{array}{l}\text { Every } 15 \text { min: } 1 \text {; per hour: } 4 \text {; every second } \\
\text { hour: } 0 \text {; if the arm is not moving: } 4 \text {; ones a day: } \\
1 \text {; none: } 0\end{array}$ \\
\hline 6 & $\begin{array}{l}\text { Should the information about the feedback be } \\
\text { send to the clinician? }\end{array}$ & Yes: 10; No: 0 \\
\hline
\end{tabular}

\section{Measurement results with the Arm Usage Coach}

All stroke subjects had hand/wrist function ${ }^{14}$ (FMA-UE distal $>8$ points, out of 30 points) and were able to done and doff the wristbands, attach the sensors to sensor holders, and mount VT actuator on the wristband, without any additional devices. In Table 3.6, a summary of the measurement results are listed (over all participants), including arm usage (in percentage of time of combined left/right arm usage) for the impaired and nonimpaired arm and the amount of VT feedback. In addition, an example of arm usage and VT feedback as shown by the AUC is shown in Figure 3.3. Each participant was able to 
Table 3.6: Summary of the measurement results.

\begin{tabular}{lcccc}
\hline & $\begin{array}{c}\text { Impaired arm } \\
\text { usage (\%) }\end{array}$ & $\begin{array}{c}\text { Non-impaired } \\
\text { arm usage (\%) }\end{array}$ & $\begin{array}{c}\text { Difference } \\
\text { impaired/ } \\
\text { non-impaired }\end{array}$ & $\begin{array}{c}\text { Amount of } \\
\text { feedback }\end{array}$ \\
\hline Average & 43 & 57 & -15 & 6 \\
Standard deviation & 24 & 23 & 47 & 2 \\
\hline
\end{tabular}

perform the measurement session (length $15 \mathrm{~min}$ ) and got VT feedback from the device (on average $6 \pm 2$ times). Overall, the non-impaired side was used in $57 \pm 23 \%$ of the time during the session compared to the impaired side with $43 \pm 24 \%$ of the time. Participants did not report to have any obstruction of the device during their activities. One participant did not feel the VT feedback during the simulated daily life activities, which was congruent with the perception impairment of the participant (Table 3.1).

\section{Postinterview}

\section{Custom questionnaire}

The results from the questionnaire given during the postinterview are listed in Table 3.7. After the measurement session, nine out of 10 participants mentioned that they like VT actuation as a feedback modality. More so, seven participants liked and found the VT feedback intuitive when the affected arm was not moving. In total, nine participants would utilize the AUC on a daily basis. All participants would share data generated by the system with a clinician. All participants indicated that they would use the AUC as an addition to

Table 3.7: Results custom questionnaire during interview 2

\begin{tabular}{|c|c|c|}
\hline \# & Question & Results \\
\hline 1 & What kind of feedback would you prefer? & Visual: 3 , acoustic: 0 , vibrotactile: 9 , none: 0 \\
\hline 2 & When should the feedback be applied? & $\begin{array}{l}\text { Every } 15 \text { min: } 0 \text {; per hour: } 3 \text {; every second hour: } \\
0 \text {; if the arm is not moving: } 7 \text {; one time per day: } \\
1 \text {; none: } 0\end{array}$ \\
\hline 3 & $\begin{array}{l}\text { Should the information about the feedback be } \\
\text { send to the clinician? }\end{array}$ & Yes: 10; No: 0 \\
\hline 4 & Would you use a device like the AUC? & Yes: $10 ;$ No: 0 \\
\hline 5 & When would you use the AUC? & Daily: 9; Weekly: 1 \\
\hline 6 & $\begin{array}{l}\text { Do you think the AUC could compliment your } \\
\text { standard therapy? }\end{array}$ & Yes: 10; No: 0 \\
\hline
\end{tabular}


their routine therapy in everyday life. Furthermore, participants liked the unobtrusiveness of the VT feedback and that the surrounding environment cannot recognize the feedback. Nine out of 10 participants found the VT feedback helpful, when they do not move the impaired arm.

\section{System Usability Scale results}

On average, patients reported a SUS score of $84( \pm 20.7)$ out of 100 points indicating excellent usability (Figure 3.4). ${ }^{12}$ Eight participants scored above 80, one between 70 and 80 , and one participant reported poor usability below 50 . This individual had the worst FMA-UE score $(\leq 40)$. Nine participants reported in the SUS that they would use the system frequently.

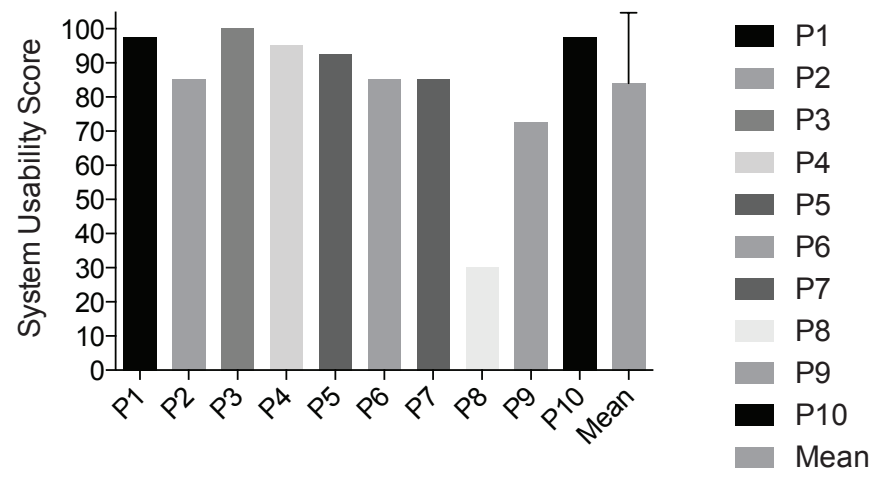

Figure 3.4: System Usability Scale results.

\section{DISCUSSION}

The objective of this study was to investigate if VT feedback is accepted and usability of the AUC in stroke subjects during a daily life activities simulation. Based on the inclusion criteria, all included stroke subjects were able to move there affected hand and were able to mount the AUC to the wrist. In total, $70 \%$ of the participants said that they would like to obtain feedback when the impaired arm was not moving during certain activities. This indicates good acceptance of the device. In total, 9 out of 10 participants were able to feel the vibration on their impaired arm. Therefore, it appears that participants accepted VT feedback. All participants reported that they would like to use the device, that it complements their current therapy, and that they prefer to share the data with a clinician. Patients agree to send the data from the AUC to a care professional to check on their progress and address this during therapy sessions, this could help to adapt the rehabilitation for upper 
extremity to the patients needs. This indicates that AUC could be used as telemonitoring and rehabilitation devices for upper extremity. The usability reported with the SUS was high, with 84 out of 100 points on average for all 10 participants. The usability of the AUC is therefore classified as excellent according to Bangor et al., 2009. ${ }^{12}$ The AUC however could, according to patients, be improved by being smaller and waterproof. It is increasingly unlikely that new usability problems will be uncovered by including more stroke subjects. ${ }^{15}$ Physical activity coaches, who mostly use accelerometers, implement different feedback strategies (in form of graphs, push-messages, VT feedback) to encourage active behaviour during daily life. ${ }^{16,17}$ In stroke, feedback is used to investigate certain interventions (e.g., constrained induced movement therapy), ${ }^{18-20}$ to correct postures of patients during specific tasks $^{21}$ or to improve motor learning capabilities. ${ }^{22}$ Moreover, many studies showed that multimodal feedback strategies, proved to be effective to improve performance of patients in various tasks and scenarios. ${ }^{23-26}$ Most of these studies are performed in a lab environment, and therefore have a reduced interest in the social context of the patients. We designed the AUC based on two feedback strategies: first, knowledge of performance, implemented by VT feedback, which is given during simulated daily life tasks in this study. The second is knowledge of results, which is given through visual feedback where the arm usage is shown in a bar graph, in percentage of the left and right arm and the number of feedbacks within a given time period. This combines the real-time VT feedback with post-visual feedback. This differs by most work done in multimodal strategies, which in most studies gives a combined (near) real-time feedback. In our design, we aimed for an unobtrusive and wearable design during daily life, without the direct need of smartphone apps to make it more applicable and intuitive for stroke patients. Acoustic feedback is obtrusive in social settings and was not implemented. Furthermore, our visual feedback needs longer data processes in order to "make sense" (arm usage); therefore, there is no need for direct visual feedback. Systems to train the upper extremity function with VT feedback in stroke patients have been previously developed. ${ }^{27-30}$ It is known that intensive training after stroke has a positive effect in clinical outcome, but the effect of VT feedback on arm function is unclear. ${ }^{30}$ The AUC could provide the opportunity to increase the arm usage in daily life, thereby training intensity and time by providing VT feedback.

We did not observe an effect of the AUC on arm usage of the impaired side. This is due to the short observation period. Because the main objective here was to test the usability and acceptance of the system, hence a short measurement time was selected.

The combination of monitoring and training stroke patients in daily life with VT feedback is new and could be realized by using a smaller, waterproofed version of the AUC. Computational tasks should be performed on the sensor, rather than on a laptop or a smartphone. 
Based on the results of this usability study, an efficacy study, with extended protocol and pre defined outcome parameter, could evaluate the impact of VT feedback on the stroke subjects arm movements in daily life activities.

\section{REFERENCES}

1. Kollen B, Kwakkel G, Lindeman E. Functional recovery after stroke: a review of current developments in stroke rehabilitation research. Reviews on Recent Clinical Trials. 2006;1(1):7580 .

2. Bussmann JBJ, Ebner-Priemer UW, Fahrenberg J. Ambulatory activity monitoring progress in measurement of activity, posture, and specific motion patterns in daily life. European Psychologist. 2009;14(2):142-152.

3. Veltink PH, van Meulen F, van Beijnum BJ, Klaassen B, Hermens HJ, Droog E, Weusthof MH, et al. Daily-life tele-monitoring of motor performance in stroke survivors. Paper presented at: International Symposium: 3D Analysis of Human Movement 3D-AHM; 2014.

4. Klaassen B, Bartels P, van Beijnum B, Hermens H. The development and evaluation of an arm usage coach for Stroke survivors. Paper presented at: 9th International Conference on Sensing Technology (ICST); 2015.

5. van Meulen FB, Klaassen B, Held J, Reenalda J, Buurke JH, van Beijnum B-JF, Luft A, et al. Objective evaluation of the quality of movement in daily life after stroke. Frontiers in Bioengineering and Biotechnology. 2016;3(210).

6. Klaassen B, van Beijnum B-J, Weusthof M, Hofs D, van Meulen F, Droog E, Luinge H, et al. A Full Body Sensing System for Monitoring Stroke Patients in a Home Environment. In: Plantier G, Schultz T, Fred A, Gamboa H, eds. Biomedical Engineering Systems and Technologies: 7th International Joint Conference, BIOSTEC 2014, Angers, France, March 3-6, 2014, Revised Selected Papers. Cham: Springer International Publishing; 2015:378-393.

7. Brook J. SUS: A Quick and dirty Usability scale. https://www.usability.gov/how-to-and-tools/ methods/system-usability-scale.html Available at. Accessed 29-12-2016.

8. Fugl-Meyer AR, Jaasko L, Leyman I, Olsson S, Steglind S. The post-stroke hemiplegic patient. 1. a method for evaluation of physical performance. Scandinavian Journal of Rehabilitation Medicine. 1975;7(1):13-31.

9. Stolk-Hornsveld F, Crow J, Hendriks E, Van Der Baan R, Harmeling-Van der Wel B. The Erasmus MC modifications to the (revised) Nottingham Sensory Assessment: a reliable somatosensory assessment measure for patients with intracranial disorders. Clinical Rehabilitation. 2006;20(2):160-172.

10. van Meulen FB, Reenalda J, Buurke JH, Veltink PH. Assessment of daily-life reaching performance after stroke. Annals of Biomedical Engineering. 2015;43(2):478-486.

11. Lyle RC. A Performance-Test for Assessment of Upper Limb Function in Physical Rehabilitation Treatment and Research. International Journal of Rehabilitation Research. 1981;4(4):483-492.

12. Bangor A, Kortum P, Miller J. Determining what individual SUS scores mean: Adding an adjective rating scale. Journal of Usability Studies. 2009;4(3):114-123.

13. Bangor A, Kortum PT, Miller JT. An Empirical Evaluation of the System Usability Scale. International Journal of Human-Computer Interaction. 2008;24(6):574-594.

14. Page SJ, Fulk GD, Boyne P. Clinically important differences for the upper-extremity FuglMeyer Scale in people with minimal to moderate impairment due to chronic stroke. Physical Therapy. 2012;92(6):791-798.

15. Virzi RA. Refining the test phase of usability evaluation: How many subjects is enough? Human Factors. 1992;34(4):457-468. 
16. Cabrita M, Nassabi MH, op den Akker H, Tabak M, Hermens H, Vollenbroek M. An unobtrusive system to monitor physical functioning of the older adults: Results of a pilot study. Paper presented at: International Workshop on Personalization and Adaptation in Technology for Health (PATH 2015); 2015.

17. Achterkamp R, Dekker-Van Weering MG, Evering RM, Tabak M, Timmerman JG, Hermens HJ, Vollenbroek-Hutten MM. Strategies to improve effectiveness of physical activity coaching systems: Development of personas for providing tailored feedback. Health Informatics Journal. 2016.

18. Harris JE, Eng JJ, Miller WC, Dawson AS. A self-administered Graded Repetitive Arm Supplementary Program (GRASP) improves arm function during inpatient stroke rehabilitation: a multi-site randomized controlled trial. Stroke. 2009;40(6):2123-2128.

19. Shi YX, Tian JH, Yang KH, Zhao Y. Modified constraint-induced movement therapy versus traditional rehabilitation in patients with upper-extremity dysfunction after stroke: a systematic review and meta-analysis. Archives of Physical Medicine and Rehabilitation. 2011;92(6):972-982.

20. Bonifer NM, Anderson KM, Arciniegas DB. Constraint-induced movement therapy after stroke: efficacy for patients with minimal upper-extremity motor ability. Archives of Physical Medicine and Rehabilitation. 2005;86(9):1867-1873.

21. Ding ZQ, Luo ZQ, Causo A, Chen IM, Yue KX, Yeo SH, Ling KV. Inertia sensor-based guidance system for upperlimb posture correction. Medical Engineering and Physics. 2013;35(2):269-276.

22. Lieberman J, Breazeal C. TIKL: Development of a wearable vibrotactile feedback suit for improved human motor learning. IEEE Transactions on Robotics. 2007;23(5):919-926.

23. Burke JL, Prewett MS, Gray AA, Yang L, Stilson FR, Coovert MD, Elliot LR, et al. Comparing the effects of visual-auditory and visual-tactile feedback on user performance: a meta-analysis. Paper presented at: Proceedings of the 8th international conference on Multimodal interfaces; 2006.

24. Prewett MS, Yang L, Stilson FR, Gray AA, Coovert MD, Burke J, Redden E, et al. The benefits of multimodal information: a meta-analysis comparing visual and visual-tactile feedback. Paper presented at: Proceedings of the 8th international conference on Multimodal interfaces; 2006.

25. Causo A, Dung TL, Yeo SH, Chen I. Visual cue and vibrotactile in series configuration: multimodal feedback design for Arm posture correction. Journal of Medical Imaging and Health Informatics. 2012;2(4):430-437.

26. Trejo-Gabriel-Galan JM, Rogel-Melgosa V, Gonzalez S, Sedano J, Villar J, Arenaza-Basterrechea $\mathrm{N}$. Rehabilitation of hemineglect of the left arm using movement detection bracelets activating a visual and acoustic alarm. Journal of Neuroengineering and Rehabilitation. 2016;13(1):79.

27. Kapur P, Premakumar S, Jax SA, Buxbaum LJ, Dawson AM, Kuchenbecker KJ. Vibrotactile feedback system for intuitive upper-limb rehabilitation. Paper presented at: EuroHaptics conference, 2009 and Symposium on Haptic Interfaces for Virtual Environment and Teleoperator Systems. World Haptics. Third Joint; 2009.

28. Acuna M, Amasay T, Karduna AR. The reliability of side to side measurements of upper extremity activity levels in healthy subjects. BMC Musculoskeletal Disorders. 2010;11(1):168.

29. Bark K, Khanna P, Irwin R, Kapur P, Jax SA, Buxbaum LJ, Kuchenbecker KJ. Lessons in using vibrotactile feedback to guide fast arm motions. Paper presented at: World Haptics Conference (WHC), IEEE; 2011.

30. Hung C-T, Croft EA, Van der Loos HM. A wearable vibrotactile device for upper-limb bilateral motion training in stroke rehabilitation: a case study. Paper presented at: Engineering in Medicine and Biology Society (EMBC), 37th Annual International Conference of the IEEE; 2015. 



\section{Chapter 4}

\section{Encouragement-induced real-world upper limb use after stroke by a tracking and feedback device: a study protocol for a multi-center, assessor-blinded, randomized controlled trial}

J.P.O. Held, A.R. Luft, J.M. Veerbeek 


\section{ABSTRACT}

Introduction Retraining the paretic upper limb after stroke should be intense and specific to be effective. Hence, the best training is daily life use, which is often limited by motivation and effort. Tracking and feedback technology have the potential to encourage self-administered, context-specific training of upper limb use in the patients' home environment. The aim of this study is to investigate post-intervention and long-term effects of a wrist-worn activity tracking device providing multimodal feedback on daily arm use in hemiparetic subjects beyond 3 months post-stroke.

Methods and analysis A prospective, multi-center, assessor-blinded, Phase 2 randomized controlled trial with a superiority framework. Sixty-two stroke patients will be randomized in two groups with a 1:1 allocation ratio, stratified based on arm paresis severity (Fugl-Meyer Assessment - Upper Extremity subscale $<32$ and $\geq$ 32). The experimental group receives a wrist-worn activity tracking device providing multimodal feedback on daily arm use for 6 weeks. Controls wear an identical device providing no feedback. Sample size: 31 participants per group, based on a difference of $0.75 \pm 1.00$ points on the Motor Activity Log - 14 Item Version, Amount of Use subscale (MAL-14 AOU), 80\% power, two-sided alpha of 0.05 , and a $10 \%$ attrition rate. Outcomes: primary outcome is the change in patient-reported amount of daily life upper limb use (MAL-14 AOU) from baseline to post-intervention. Secondary outcomes are change in upper limb motor function, upper limb capacity, global disability, patient-reported quality of daily life upper limb use, and quality of life from baseline to post-intervention and 6-week follow-up, as well as compliance, activity counts, and safety.

Discussion The results of this study will show the possible efficacy of a wrist-worn tracking and feedback device on patient-reported amount of daily life upper limb use.

Ethics and dissemination The study is approved by the Cantonal Ethics Committees Zurich, and Northwest and Central Switzerland (BASEC-number 2017-00948) and registered in https://clinicaltrials.gov (NCT03294187) before recruitment started. This study will be carried out in compliance with the Declaration of Helsinki, ICH-GCP, ISO 14155:2011, and Swiss legal and regulatory requirements. Dissemination will include submission to a peer-reviewed journal, patient and healthcare professional magazines, and congress presentations. 


\section{INTRODUCTION}

Although medical interventions and care for stroke patients have been improving tremendously during the last decades, stroke still remains one of the main causes of disease burden globally. ${ }^{1,2}$ Frequently reported limitations after stroke are impairments of upper limb motor function and activities, which is present in $80 \%$ of the acute stroke patients. ${ }^{3,4}$ Recovery of post-stroke impairments mainly occurs within the first 3 months after stroke and plateaus thereafter. ${ }^{5,6}$ Although the presence of a plateau phase implies that patients have reached a stable situation, a functional decline has been shown on the long term (i.e., learned-non-use). ${ }^{7}$ This underlines the importance of continued practice beyond the first 3 months post-stroke, with the aim to maintain the levels achieved during earlier rehabilitation. ${ }^{8}$

Key elements that characterize effective stroke rehabilitation interventions are intensity of practice, and task- and context-specificity. ${ }^{9,10}$ Provision of feedback is another important ingredient for effective motor learning after stroke. ${ }^{11}$ In the last decade, rehabilitation technology has enabled higher intensity of practice and new methods of feedback. Examples of applied technologies for the upper limb are robotics and virtual reality. ${ }^{12,13}$ However, these interventions often lack context-specificity, as the training is almost always performed in a clinic and to date, a beneficial effect on what patients actually do with their paretic upper limb in their daily lives has not been reported. Furthermore, many of these technological developments require a high-financial investment in terms of costs and human resources. Recent developments in the field of tracking and feedback technology have provided low-cost tools (e.g., sensors) that incorporate key elements for effective stroke rehabilitation outside the clinic. These tracking and feedback tools have the potential to motivate patients to use their paretic upper limb in daily life while they are in their home environment. With that, they possibly are able to maintain or even improve gains made during the intensive rehabilitation period and facilitate a translation to the patients' daily life. These tools most often rely on accelerometer data, which have been found to be reliable and valid in stroke subjects, ${ }^{14,15}$ and correlate well with patient-reported upper limb use in daily life as assessed by the Motor Activity Log. ${ }^{16}$ Previous research has shown that stroke survivors have a high adherence to wearing accelerometer devices at hom $\mathrm{e}^{17}$ and report good acceptance levels and high usability scores. ${ }^{18,19}$ These tracking devices can be used to give augmented feedback regarding movement outcomes or success rate (i.e., knowledge of results). ${ }^{20,21}$ Knowledge of results is an external form of feedback that can encourage a patient to reach a certain goal, such as using the paretic upper limb at least a pre-defined number of times a day (e.g., 100) when performing daily life activities. In this example, the external feedback given by the trackers could enhance a patient's intrinsic motivation to 
improve the number of times he or she uses the upper limb during the day. The provided feedback should be tailored to the individual needs to increase motivation, compliance, and effectiveness. There are various feedback modalities available, such as vibrotactile, visual, and auditory feedback. A recent study reported that stroke patients preferred vibrotactile feedback over visual and auditory feedback. ${ }^{19}$ However, the application of visual feedback on a smartphone has also been indicated as an effective way of delivering feedback. ${ }^{22}$ With that, the optimal medium for providing feedback remains unknown.

\section{Study aim}

The primary aim of this study is to determine the post-intervention and long-term effects of wearing a wrist-worn, commercially available tracking device that provides multimodal feedback for 6 weeks on patient-reported amount of paretic upper limb use in daily life in hemiparetic subjects beyond 3 months after stroke, when compared with a control group receiving an identical looking device providing no feedback. The secondary aims are to examine the compliance to use the device, and explore the effect on motor function of the paretic upper limb, upper limb capacity, patient-reported quality of paretic upper limb use in daily life, global disability, health-related quality of life, and during the 6-week intervention, the compliance and activity counts of the paretic and non-paretic upper limb. As a measure of safety, the incidence and severity of side effects related to the long-time wrist-worn feedback intervention during the study period will be investigated. Furthermore, the Minimal Clinical Important Difference (MCID) of the Amount of Use (AOU) subscale of the Motor Activity Log - 14 Item Version (MAL-14) will be established.

\section{METHODS AND ANALYSIS}

\section{Study design}

The present study is a multi-center, assessor-blinded, Phase 2 randomized controlled trial with a superiority framework, including two parallel study arms. Patients will be informed about the study, including its procedures, and enrolled by a study team member. Randomization will be stratified based on severity of upper limb paresis (Fugl-Meyer Assessment - Upper Extremity subscale (FMA-UE) $<32$ and $\geq 32)^{23}$ with a 1:1 allocation ratio. At the time of patient registration in the centralized web-based database (REDCap ${ }^{\mathrm{TM}}$ ), patients will be given a unique study identification number that is linked to a computergenerated randomization assignment using a pre-set list of random numbers. The pre-set list of random numbers was generated based on a seed number $\left(\mathrm{SAS}^{\varpi} 9.3\right)$ and balanced 
based on the stratification in blocks of 4 . Randomization is performed by a study member not involved in the outcome assessments.

An Emergency Code Break will be available to the investigator. This Code Break should only be opened in emergency situations when the identity of the investigational product must be known by the investigator in order to provide appropriate medical treatment.

This study was approved by the Cantonal Ethics Committee Zurich and Cantonal Ethics Committee Northwest and Central Switzerland (BASEC-number 2017-00948). All subjects will give written informed consent in accordance with the Declaration of Helsinki. The trial is registered at https://clinicaltrials.gov, unique identifier NCT03294187 prior to patient recruitment.

\section{Eligibility criteria / participants}

This study includes subjects aged 18 years or older who have experienced a unilateral stroke more than 90 days ago and have residual upper limb paresis after completion of all inpatient rehabilitation. Subjects have to be able to lift the paretic arm against gravity $\left(>30^{\circ}\right.$ of flexion or abduction), don/doff the devices on both wrists independently or with the assistance of a caregiver, and provide informed consent as documented by signature. Patients will be excluded from this study if they have major untreated depression, severe cognitive impairment, comprehensive aphasia, and/or severely impaired sensation (unable to feel a soft touch on the dorsal side of their paretic wrist with closed eyes). Other exclusion criteria are a potential non-compliance such as hospitalization during the study period, known intolerance to the device material, known drug or alcohol abuse, and/or other major comorbidities (e.g., cardiopulmonary disease, renal failure, hepatic dysfunction, and orthopedic disorders of the upper limb).

\section{Stepwise procedures}

Subjects will be recruited at three study centers in Switzerland: University Hospital Zurich (academic hospital), cereneo - Center for Neurology and Rehabilitation (rehabilitation clinic), and Zürcher RehaZentrum Wald (rehabilitation clinic) by the local principal or sub-investigator. The participant timeline is displayed in Figure 4.1. The written participant information and informed consent forms (in German) can be obtained by the corresponding author. After having obtained participant consent, the baseline assessment (T0) will be performed. Randomization will take place after the baseline assessment and depending on the group allocation, participants will be handed over the trial arm-specific devices and receive instructions accordingly. Randomization and device hand-over will be performed by study 


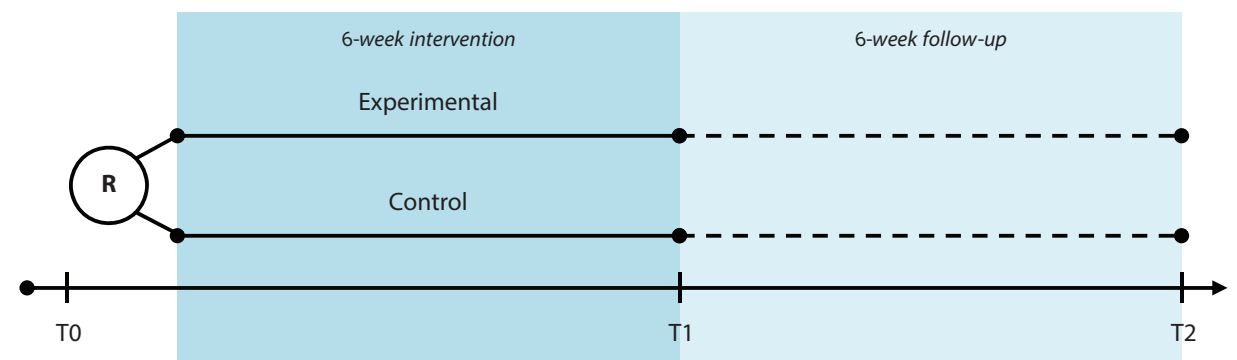

Figure 4.1: Participant time line.

Legend: R, Randomization; T0, Baseline assessment; T1, Post-intervention assessment; T2, Follow-up assessment.

personnel not involved in assessments (e.g., physical therapist, study nurse). The intervention period has a total duration of 6 weeks. The post-intervention assessment (T1) takes place at day $45 \pm 3$ after baseline and the follow-up assessment (T2) at day $90 \pm 6$ after baseline.

Recruitment started in September 2017 and is expected to last until December 2018, which means that the estimated study completion will be in March 2019. If inclusion stays behind, ethical approval will be obtained for adding other study centers in order to enroll the required number of patients within this timeframe. No payment will be provided to the participants other than compensation for travel costs.

\section{Interventions}

The investigational devices to be used in the study are (see Figure 4.2):

- Motion tracker "ARYS ${ }^{\mathrm{TM}}$ me $\mid$ tracker" (Figure 4.2A: black-silver tracker).

- Motion tracker "ARYS ${ }^{\mathrm{TM}}$ pro| tracker healthy" (Figure 4.2B: black-brown tracker).

Additionally, the patients receive:

- Two accessory charging stations “ARYS ${ }^{\mathrm{TM}}$ | tracker charger" (Figure 4.2C).

- An Android smartphone with the preinstalled Application "ARYS ${ }^{\mathrm{TM}}$ me |app"; only for study subjects in the intervention group (Figure 4.2D); and

- A manual covering the following topics: study system components, charging the trackers, placement of the trackers, when not to use the trackers (e.g., bathing, swimming, MRI scanning, and uncomfortable feeling on the wrist), Frequently Asked Questions regarding technical problems and cleaning, and contact information. Additionally, the manual for the experimental group provides information regarding on-device feedback and reminders, use of the smartphone app, and daily goals. 
A

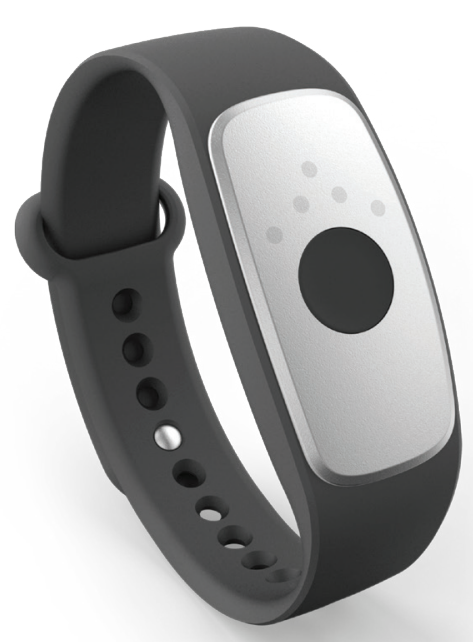

C

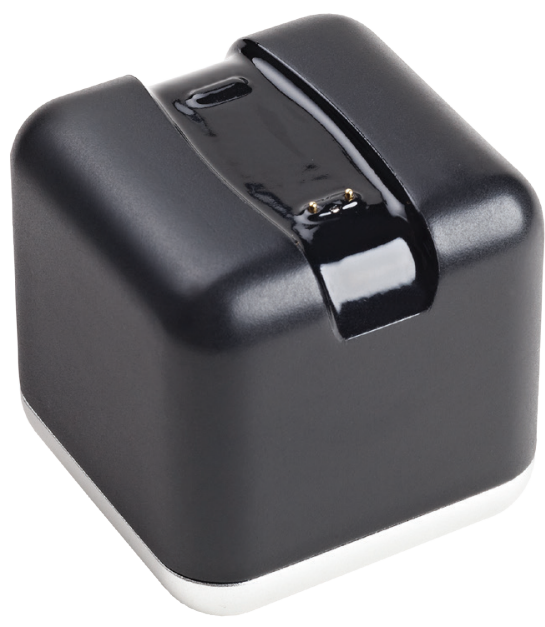

B

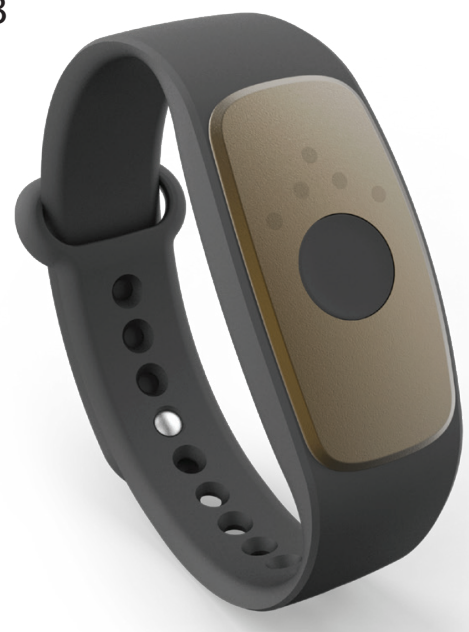

D

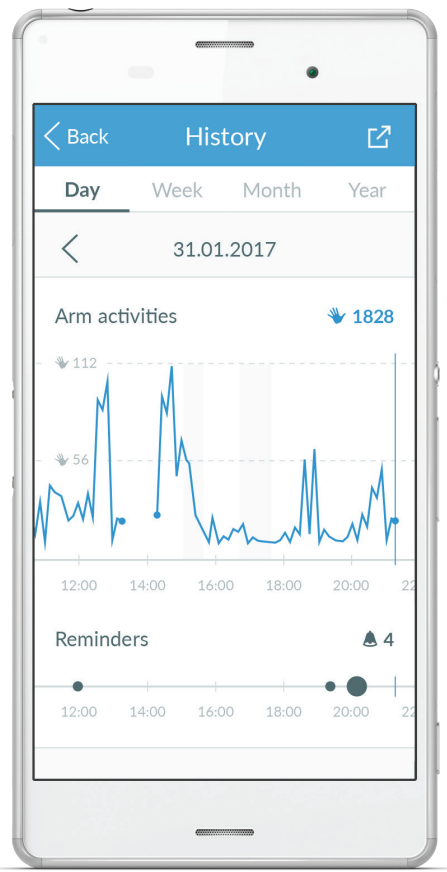

Figure 4.2: Investigational devices.

A) Motion tracker "ARYS ${ }^{\mathrm{TM}}$ me $\mid$ tracker" black-silver tracker; B) "ARYSTM pro | tracker healthy" black-brown tracker; C) Accessory charging station "ARYS"M $\mid$ tracker charger"; D) Android smartphone with the preinstalled Application "ARYS ${ }^{\mathrm{TM}}$ me $\mid$ app", here displaying the history of today's Arm Activities, including provided reminders. 
The devices are manufactured and distributed by "yband therapy AG" and are intended for use in arm therapy of patients with arm movement deficiencies. The arm movement deficiencies may have been caused by cerebral, neurogenic, and spinal-related disorders. The "ARYS ${ }^{\mathrm{TM}}$ me $\mid$ tracker" and "ARYS ${ }^{\mathrm{TM}}$ pro $\mid$ tracker healthy" are both CE-certified as a "Class 1 Medical Device".

\section{Experimental intervention}

Participants in the experimental group will wear an "ARYS ${ }^{\mathrm{TM}}$ me $\mid$ tracker" on the paretic wrist and an "ARYS ${ }^{\mathrm{TM}}$ pro | tracker healthy" on the non-paretic wrist (see Figure 4.2). The trackers are hardware-wise identical and consist of: the actual tracker (silver with a feedback and communication module for paretic side, which is deactivated for the control group), a reference tracker (black brown tracker for the non-paretic side); and an exchangeable, flexible black wristband. Both elements are made out of biocompatible materials. The devices should be worn every day, as long as possible over a period of 6 weeks. During this 6-week period, a three-axis accelerometer in each device will constantly monitor the subjects' arm movements. A threshold of $0.1 \mathrm{~g}$ acceleration is defined for detection of movements. This raw acceleration data, aggregated over 1 minute, will be converted on-device in so-called "Arm Activities". Starting from a pre-defined goal-value, new daily goals will be automatically calculated every day from the rolling average of the last 30 days plus 3\% (e.g., after the first intervention week, patient X shows an average number of 100 "Arm Activities" per day. For day 8, his daily goal therefore becomes 103 "Arm Activities"). Patients will constantly be challenged to slightly increase their Arm Activities without ever being overstrained. Assuming a steady arm activity during the course of the day, a linear target activity line will be calculated (default start/end times: 8:00 a.m. to 10:00 p.m.). The already reached "Arm Activities" will be compared with an intermediate target value. After 30 minutes of inactivity, the "ARYS ${ }^{\mathrm{TM}}$ me $\mid$ tracker" vibration engine will provide the subject on the paretic wrist with two short double pulses and up to four LEDs will light up in orange color to remind to make more use of this arm (Level 1 Reminder). If $30 \mathrm{~min}$ later, "Arm Activities" are still below the intermediate target, a second escalation level of the reminder is triggered: five long vibrating pulses will be provided and up till four LEDs will light up in red color (Level 2 Reminder). Thereafter, the escalation level is reset and starts over again with the Level 1 reminder next time. Additionally, by pressing the button on the "ARYS ${ }^{\mathrm{TM}}$ me $\mid$ tracker" once, the LED lights will show the percentage of the daily target that is already reached (one white LED $=<25 \%$; two white LEDs $=25-50 \%$; three white LEDs $=50-75 \%$; three white LEDs and one blinking LED = 75-99\%; four white LEDs $=100 \%$, daily target reached) and the patient receives vibration feedback. When the patient has reached the daily target, the tracker vibrates. 
Participants in the intervention group are encouraged to regularly check the pre-installed "ARYS ${ }^{\mathrm{TM}}$ me app" on a standard commercial Android smartphone, which they have received. It will download "Arm Activity" data from the "ARYS ${ }^{\mathrm{TM}}$ me $\mid$ tracker" automatically, motivate study subjects based on the concepts of gamification, and visualize activity data over various time intervals. Patients can monitor the development of their daily targets, today's progress, and provided reminders towards the daily target, as well as a history of all past "Arm Activities" and reminders presented in a day, week, month, and year view (see Figure 4.2D). Additionally, they can follow the growth of the "Tree of Recovery" (see Figure 4.3). This is a figurative representation of their amount of upper limb use over time, in which they earn "Diamonds" by fulfilling challenges of intensive activity and can use these "Diamonds" to water their tree to let it grow and flourish.

For using the devices, no medical and/or surgical procedures will be involved. However, a tight fit on the wrist will be beneficial to reduce motion artifacts and to ensure that the vibration feedback is properly felt by the study subjects. As both devices are splash-
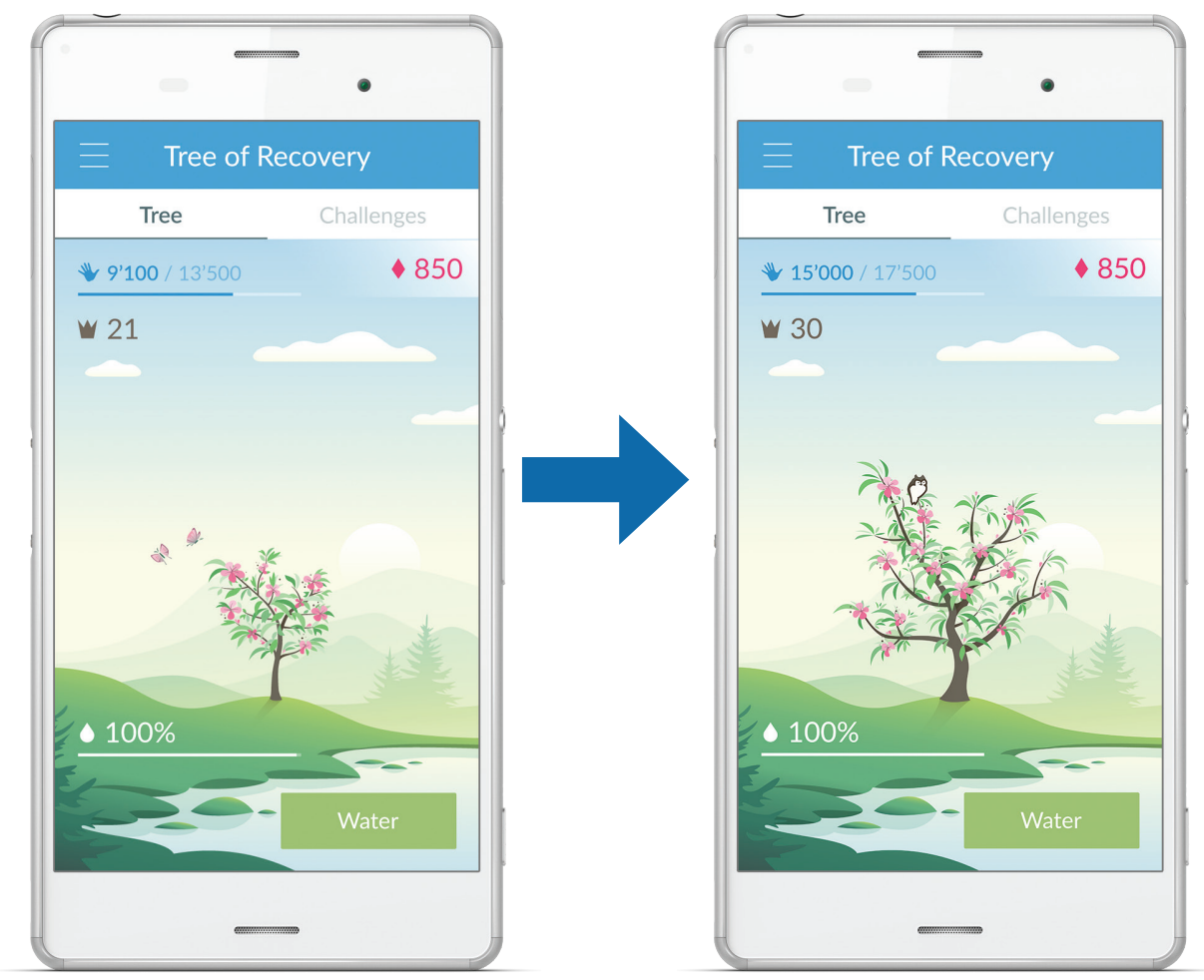

Figure 4.3: "Tree of Recovery".

A figurative representation of the amount of upper limb use in daily life. Arm Activities are displayed in blue numbers and the earned diamonds in red. 
waterproof, there is no need to doff the trackers during daily activities involving water, such as washing hands or showering. Thanks to the easy-to-use pin-and-tuck closure, study subjects should be able to don/doff the device on their own without any help (see Figure 4.2A, B). Other than instruction through a non-blinded study member at one of the participating study sites and the information provided in the group-specific leaflet, no training or experience will be required to use the devices. The one-to-one instruction takes up to 30 minutes. Furthermore, the patients can always look up information in the accompanying manual, pose questions during the weekly telephone calls, and call the hotline.

\section{Control intervention}

Subjects in the control group will use the same devices in the same way as the intervention group. They will wear the "ARYS ${ }^{\mathrm{TM}}$ me $\mid$ tracker" on their paretic and the "ARYS ${ }^{\mathrm{TM}}$ pro | tracker healthy" on their non-paretic wrist. The "ARYS ${ }^{\mathrm{TM}}$ me $\mid$ tracker" will have a custom firmware installed that deactivates both the vibration module as well as the LEDprogress bar. Therefore, subjects in the control group will neither receive nor know about any feedback happening in the intervention group. They will not receive a smartphone with the "ARYS ${ }^{\mathrm{TM}}$ me $\mid$ app". The one-to-one instruction for the control intervention takes up to 15 minutes. Analogue to the patients in the experimental group, they can consult the accompanying manual, ask questions during the weekly telephone calls, and, when needed, contact the hotline.

\section{Additional information regarding the interventions}

To increase study compliance and adherence, both intervention and control group subjects will receive weekly reminder calls (six in total) to wear the "ARYS ${ }^{\mathrm{TM}} \mid$ tracker" devices every day. These calls will be performed by an unblinded study team member. Additionally, there will be a hotline telephone number they can call when facing any technical problems. Discontinuation of the allocated intervention for a given trial participant will take place in case of, for example, occurrence of a Serious Adverse Event (AE) or participant request. We expect no modifications on the given intervention during the study. All participants are permitted to receive concomitant rehabilitation interventions such as physical therapy and/or occupational therapy during the trial. The time spent in these interventions will be registered during the total study period.

\section{Sample size}

A sample size calculation based on a difference between groups in change from baseline to post-intervention in the AOU subscale of the MAL-14 of 0.75 points. With an effect 
SD of 1.00 , a two-sided alpha level set at 0.05 , and a power of $80 \%, 28$ subjects per group are required. ${ }^{24}$ Taking into account an attrition rate of $10 \%$, a total of 62 subjects will be randomized. In case of an attrition rate higher than the expected $10 \%$, post-recruitment for replacement will take place.

\section{Measures}

Potential participants will be screened and examined by the local study team for eligibility. Included subjects will be assessed prior to randomization (T0), after the 6-week intervention (T1), and 6 weeks thereafter (T2). The outcome assessors will be trained regarding the assessments and are blinded to group allocation. Study participants will be told at the beginning of the study, as well as right before and during the T1 and T2 assessments, not to mention any details regarding their experiences with the devices during the assessment visits. Potential success or failure of blinding will be checked, by asking the assessors at the end of the post-intervention and follow-up visits, in which group they think a given patient was allocated. Success of blinding will be presented in a descriptive manner.

Primary endpoint is the change from T0 to T1. An overview of the measures and timing of assessments is displayed in Table 4.1. Data collection forms (in German) can be obtained by the corresponding author upon request.

\section{Primary outcome}

The primary outcome measure of the present study is the patient-reported amount of use of the paretic upper limb in daily life, measured with the MAL-14 AOU subscale. ${ }^{24-26}$ In a semi-structured interview, the patient is questioned regarding how often he or she has used the paretic upper limb during 14 activities of daily living (ADL) tasks on a six-point ordinal scale. For each item, the score ranges from 0 (did not use my weaker arm [not used]) to 5 (used my weaker arm as often as before the stroke [same as pre-stroke]. Scores are only given if the patient has performed the ADL task during the last week. The total score for the MAL14 AOU subscale ranges from 0 to 5 , calculated by adding scores for each of the performed items and subsequently dividing this number by the total number of performed tasks.

\section{Secondary outcomes}

The secondary outcome measures include the FMA-UE, Action Research Arm Test (ARAT), EuroQol Five Dimensions Five Levels Questionnaire (EQ-5D-5L), modified Rankin Scale (mRS), MAL-14 Quality of Movement (QOM) subscale, Global Rating of Perceived Changes (GRPC), Quantitative Sensor Data for compliance and Arm Activities of the paretic and non-paretic upper limb during the 6-week intervention, and AEs. 
Table 4.1: Overview of study measures

\begin{tabular}{|c|c|c|c|c|c|}
\hline Measure & Domain measured & T0 & I & T1 & $\mathrm{T} 2$ \\
\hline \multicolumn{6}{|l|}{ Primary outcome } \\
\hline $\begin{array}{l}\text { Motor Activity Log - } 14 \text { Item Version } \\
\text { (Amount of Use) }\end{array}$ & $\begin{array}{l}\text { Patient-reported amount of } \\
\text { upper limb use in daily life }\end{array}$ & $X$ & & $x$ & $x$ \\
\hline \multicolumn{6}{|l|}{ Secondary outcomes } \\
\hline $\begin{array}{l}\text { Motor Activity Log - } 14 \text { Item Version } \\
\text { (Quality of Movement) }\end{array}$ & $\begin{array}{l}\text { Patient-reported quality of } \\
\text { upper limb use in daily life }\end{array}$ & $x$ & & $x$ & $x$ \\
\hline Fugl-Meyer Assessment - Upper Extremity & Upper limb motor function & $\mathrm{X}$ & & $\mathrm{X}$ & $x$ \\
\hline Action Research Arm Test & Upper limb capacity & $X$ & & $X$ & $x$ \\
\hline $\begin{array}{l}\text { EuroQol Five Dimensions Five Levels } \\
\text { Questionnaire }\end{array}$ & Health-related quality of life & $\mathrm{X}$ & & $\mathrm{X}$ & $\mathrm{x}$ \\
\hline modified Rankin Scale & Global disability & $x$ & & $x$ & $x$ \\
\hline Adverse Events & Safety & $\mathrm{X}$ & $X$ & $\mathrm{X}$ & $\mathrm{X}$ \\
\hline Global Rating of Perceived Changes & Self-perceived change & & & $\mathrm{X}$ & $\mathrm{X}$ \\
\hline Quantitative Sensor Data & $\begin{array}{l}\text { Compliance; activity counts } \\
\text { of the paretic and non-paretic } \\
\text { side }\end{array}$ & & $x$ & & \\
\hline \multicolumn{6}{|l|}{ Other measures } \\
\hline Demographics & Participant demography & $X$ & & & \\
\hline Stroke Event Data & Disease characteristics & $x$ & & & \\
\hline Charlson Comorbidity Index & Medical history & $x$ & & & \\
\hline National Institutes of Health Stroke Scale & Neurological impairments & $\mathrm{X}$ & & & \\
\hline Edinburgh Handedness Inventory & Handedness & $\mathrm{X}$ & & & \\
\hline Apples Test & Visuospatial neglect & $\mathrm{X}$ & & & \\
\hline Concomitant Therapy & $\begin{array}{l}\text { Standard rehabilitation } \\
\text { therapy }\end{array}$ & $X$ & & $X$ & $x$ \\
\hline
\end{tabular}

I, Intervention; T0, Baseline; T1, Post-intervention; T2, 6-week Follow-up.

\section{Fugl-Meyer Assessment - Upper Extremity Subscale}

The FMA-UE measures motor function of the paretic upper limb. ${ }^{27,28} \mathrm{~A}$ total of 33 items are assessed and each item is rated on a three-point ordinal scale $(0=$ cannot perform, 1 $=$ performs partially, 2 = performs fully). The sum score ranges from 0 to 66 .

\section{Action Research Arm Test}

Upper limb capacity is measured with the ARAT and includes four categories: grasp (6 items), grip (six items), Pinch (six items) and gross movement (three items) ${ }^{29-31}$ Each item is rated on a four-point ordinal scale ( $0=$ no movement; $1=$ the movement task is partially performed; 2 = the movement task is completed, but takes abnormally long; and $3=$ the movement is performed normally), leading to a maximum overall score of 57 points. 


\section{EuroQol Five Dimensions Five Levels Questionnaire}

The EQ-5D-5L is a self-completed questionnaire regarding health-related quality of life. ${ }^{32,33}$ Each of the five assessed domains (mobility, self-care, usual activities, pain/discomfort, and anxiety/depression) is described by one out of five responses, ranging from "no problem/ not at all" to "unable/major problem". In addition, the patient is asked to rate his or her self-rated health on a Visual Analogue Scale (VAS). Approval of the EuroQol Research Foundation to use the German (Switzerland) EQ-5D-5L paper version has been obtained (March 13, 2017).

\section{Modified Rankin Scale}

Global disability is assessed with the mRS, which includes an informal interview with the patient, to rate the extent of a patient's post-stroke disability or impairment in ADLs on a six-point ordinal scale, ranging from $0=$ "no symptoms at all" to $5=$ "severe disability" ${ }^{4-36}$

\section{Motor Activity Log -14 Item Version - Quality of Movement Subscale}

The MAL-14 QOM measures the patient-reported quality of paretic upper limb use in daily life by performing a semi-structured interview. ${ }^{24-26}$ Analogue to the AOU subscale, 14 ADL tasks are questioned. Patients score how well the paretic upper limb helped during this activity on a six-point ordinal scale, with item scores ranging from 0 (My weaker arm was not used at all for that activity [not used]) to 5 (The ability to use my weaker arm for that activity was as good as before the stroke [normal]), and a total score ranging from 0 to 5 .

\section{Global Rating of Perceived Changes}

With the GRPC, the patients rate their perceived changes in the daily life usage of the paretic upper limb, using the following seven-point Likert scale: score 1 is much better; score 2 = a little better, meaningful; score 3 = a little better, not meaningful; score $4=$ about the same; score 5 = a little worse, not meaningful; score 6 = a little worse, meaningful; and score $7=$ much worse. ${ }^{37}$

\section{Quantitative Sensor Data for Compliance and Activity Counts}

Adherence will be monitored through the weekly reminder calls and through analysis of the gathered Quantitative Sensor Data and smartphone App usage data. The Quantitative Sensor Data measure the "Arm Activities" by using the acceleration data, aggregated over 1 min during wearing the activity tracker. This refers to Arm Activities of both the paretic and non-paretic side and allows comparing both changes in Arm Activities over time for each upper limb during the 6-week intervention, as well as comparing Arm Activities between the paretic and non-paretic upper limb. 


\section{Safety}

Adverse Events (AEs) will be gathered from inclusion upon follow-up. Assessments will be made during each study visit, as well as by the weekly phone calls during the intervention period. Each time, study subjects will be actively questioned by unblinded study personnel whether they experienced any AEs. In case of occurrence of a related AE, the time of onset, the duration, the resolution, actions that were taken, the intensity as well as the relationship with the study intervention will be recorded.

\section{Descriptive measures}

Next to the above described outcome measures, the following data will be collected at baseline to characterize our cohort: demographic data, stroke event data, comorbidities, handedness (Edinburgh Handedness Inventory $[\mathrm{EHI}]^{38}$ ), neglect (Apples Test $\left.[\mathrm{AT}]\right]^{39}$ ), social situation, and neurological functions (National Institutes of Health Stroke Scale [NIHSS $]^{40}$ ).

\section{Data collection}

Assessments will take place at one of the participating centers, or at the patients' home when he or she is not able to visit one of the study centers. All assessors will be trained prior to the first screening during which they will receive proper instruction and guidance regarding all outcome parameters and assessments that will be taken. The trial management committee (TMC) will be available for questions.

In the case that a patient discontinues or deviates from the intervention protocol, all efforts will be made to obtain at least the primary outcome measure (MAL-14 AOU) and the safety measures (AEs) at the pre-defined study visits. All patients will receive a reminder for each visit to diminish retention and incomplete follow-up.

\section{Data management}

Study data will be recorded in electronic Case Report Forms (eCRF). For each enrolled study participant, an eCRF will be maintained. These eCRFs will be kept current to reflect subject status at each phase during the course of study. The participants' code will be assigned in a random order. Source data will be available at the respective study site to document the existence of the study participants. Source data will include the original documents relating to the study, as well as the medical treatment and medical history of the participant. All study data will be archived for a minimum of 10 years after study termination or premature termination of the clinical trial in a secure database. Study data will be managed using REDCap ${ }^{\mathrm{TM}}$, being hosted on servers administered by the Data Informatics Services 
Core of cereneo Schweiz AG. To access the REDCap ${ }^{\mathrm{TM}}$ site, all study team users of the REDCap $^{\mathrm{TM}}$ system will be issued a unique username and password that is generated and maintained by our administrator on the REDCap ${ }^{\mathrm{TM}}$ server.

The study data will be analyzed by the TMC after study completion (last patient, last visit). No interim analysis will be performed. Only the study coordinators and principle investigators will have access to the final trial dataset.

\section{Statistical analysis}

Patient demographics and baseline data will be analyzed by trial arm using summary descriptive statistics. Baseline differences between groups will be tested by the independentsamples t-test for parametric data, Pearson's chi-squared test for categorical data, and the Mann-Whitney $U$ test for non-parametric data.

Differences in change scores (T0-T1) between groups will be analyzed for the primary outcome (MAL-14 AOU) and secondary outcomes (MAL-14 QOM, FMA-UE, ARAT, EQ-5D-5L, mRS, Quantitative Sensor Data). In addition, change will be monitored in the follow-up assessments (T1-T2). An analysis of covariance (ANCOVA) will be applied, with the baseline value of the measure of interest as a covariate. Compliance to using the devices during the 6-week intervention period and safety data will be presented by trial arm with descriptive statistics and compared between groups by the independent-samples t-test, Pearson's chi-squared test, or Mann-Whitney $U$ test, depending on the nature of the data distribution. In a secondary analysis, compliance to wearing the trackers - based on the Quantitative Sensor Data - will be added as a covariate for analysis of the primary outcome MAL-14 AOU.

All randomized subjects will be included in the analyses according to the intention-to-treat approach. Missing data will be imputed. In addition, differences in baseline characteristics of patients who did and did not dropout will be formally tested. A p-value of $<0.05$ will be considered to be statistically significant. The analyses will be performed in IBM SPSS Statistics 23.

Furthermore, the compliance to the intervention will be presented with descriptive statistics. The MCID will be calculated based on the MAL-14 AOU and GRPC scores, analogue to previous work by Lang et al. ${ }^{37}$ 


\section{Monitoring and quality control}

The sponsor-investigator has the overall responsibility for the study conduct. The TMC at the University Hospital Zurich provides day-to-day support for the sites.

Regular monitoring visits at the investigator's site prior to the start and during the course of the study will help to follow-up the progress of the clinical study, to assure utmost accuracy of the data, and to detect possible errors at an early time point. All original data including patient files, progress notes, and copies of laboratory and medical test results will be available for monitoring. The monitor will review all or a part of the eCRFs and written informed consent forms. The accuracy of the data will be verified by reviewing the above referenced documents. A formal Data Monitoring Committee (DMC) or equivalent body will not be convened, as the study is approved by the ethical committees as a clinical trial with a CE-marked Medical Device (MD) with minimal risks. However, safety data will prospectively be reviewed at monthly TMC meetings.

The entered data will be verified by an independent unblinded study nurse, after a block of 10 subjects have been enrolled in the study.

\section{Audits and inspections}

A quality assurance audit/inspection of this study may be conducted by the Competent Authority or Competent Ethics Committee, respectively. The quality assurance auditor/ inspector will have access to all medical records, the investigator's study-related files and correspondence, and the informed consent documentation that is relevant to this clinical study. The investigator will allow the persons being responsible for the audit or the inspection to have access to the source data/documents and to answer any questions arising. All involved parties will keep the patient data strictly confidential.

The primary endpoint (MAL-14 AOU) and AEs will be monitored by the TMC.

\section{Anticipated results}

We expect that a 6-week program of wearing a wrist-worn activity tracking device, providing multimodal feedback on daily life arm use will induce statistically significant and clinical relevant changes in daily life upper limb use, when compared to wearing a sham device in patients who are beyond 3 months post-stroke. 


\section{DISCUSSION}

By applying wrist-worn activity tracking and feedback devices, this study aims to optimize patient-reported amount of daily life upper limb use in stroke survivors beyond the first 3 months after stroke onset. Patients are eligible, regardless whether they have suffered an ischemic or hemorrhagic stroke. Although it has been shown that hemorrhagic stroke patients are more severely affected in the acute stage when compared to ischemic stroke patients, ${ }^{41}$ long-term functional outcomes were not significantly different. ${ }^{41,42}$ As we include stroke survivors beyond 3 months post-stroke, we do not expect that type of stroke affects the outcomes of the present study. In addition, since we will recruit stroke patients beyond 3 months after stroke onset, we do expect that recovery has plateaued and no improvements will occur in the absence of intensive rehabilitation.

Previous studies have shown that intensive rehabilitation can influence upper limb capacity in chronic stroke patients, but context-specificity is often limited and the observed improvements fail to translate to upper limb use in daily life. ${ }^{10}$ The present study can help to close the gap that exists regarding the translation of gains made during inpatient rehabilitation to the home situation. The amount that patients use their paretic arm in daily life could be enhanced by providing multimodal feedback when they are in their home environment. Feedback can be used to motivate stroke patients and with that, positively impact patient outcomes. ${ }^{43}$ The experimental trial arm provided feedback regarding Arm Activities (i.e., knowledge of results). This can mainly be seen as extrinsic feedback, as the patients receive immediate vibrotactile feedback from the trackers when paretic arm use is below the pre-set daily target (i.e., controlled motivation). They furthermore receive delayed feedback regarding paretic arm use when they use the smartphone applcation. However, the use of the pre-installed application on the smartphone also requires selfdetermined motivation (i.e., self-control of feedback) of the patient, as he or she actively has to open the application for checking their progress and play the "Tree of Recovery". We expect that the combination of various forms of feedback (vibrotactile feedback, visual feedback by the LED lights, information regarding upper limb activity counts in the app, and gamification) increases patient-reported amount of upper limb use in daily life, which consolidates at follow-up.

The applied tracking and feedback device focuses on the number of times that the patient moves the paretic upper limb in daily life. The MAL-14 AOU as a primary outcome matches this goal, by asking patients to rate how often they have used their paretic upper limb in daily life activities. We hypothesize that the change in MAL-14 AOU from baseline to post-intervention and follow-up will significantly differ between the experimental and 
control groups, favoring the experimental group. We expect that this difference will be also clinically relevant. This means that the change observed in the experimental group from baseline to post-intervention is at least 0.75 points more when compared to the control group. The latter is especially important, as clinical applicability and acceptability are highly dependent on whether a statistically significant difference is also meaningful for clinical practice. Although this study is not powered for our secondary outcomes, we expect to see relevant differences between groups regarding upper limb capacity (ARAT) and changes in upper limb activity counts as measured by the tracker devices itself during the 6-week intervention. Contrary, we do not expect to find clinically relevant change on motor function of the paretic upper limb (FMA-UE). First of all, because the feedback does not relate to QOM. Second, while patients in the chronic phase usually do not show significant, clinically relevant changes on motor function beyond 3 months post-stroke. ${ }^{44,45}$ Furthermore, we do not expect to see a difference between groups regarding safety and compliance to wear the devices.

A positive result of this study will underline the importance of tracking and feedback on daily life upper limb use after stroke. It would provide therapists with a tool to enhance their patients' real-world upper limb use, without being labor-intensive. Furthermore, the application of a tracking and feedback device could potentially reduce healthcare costs, although that is something that is beyond the scope of the present study.

\section{ETHICS AND DISSEMINATION}

This study will be carried out in compliance with the protocol approved by the previously mentioned ethical committees (version 1.1, dated 20/07/2017), the current version of the Declaration of Helsinki, the ICH-GCP or ISO 14155:2011, as well as all Swiss national legal and regulatory requirements. All subjects will give written informed consent in accordance with the Declaration of Helsinki. The trial is registered at https://clinicaltrials.gov, unique identifier NCT03294187. When protocol amendments are needed (e.g., to include another participating center), ethical approval will be obtained first. After having obtained this approval, relevant adaptations will be made in the relevant clinical trial registry databases.

Potentially eligible participants will be screened by the study site principal or subinvestigator for the presence of a first stroke $>90$ days, their ability to lift the paretic arm against gravity ( $>30^{\circ}$ flexion or abduction), to don/doff the "ARYS ${ }^{\mathrm{TM}} \mid$ tracker" devices independently on both wrists or if not, whether a caregiver is at hand for assistance, and to feel a soft touch on the dorsal side of their paretic wrist with closed eyes. Additionally, potential participants will be questioned and their medical record will be checked in 
regard to the other eligibility criteria. Given eligibility to take part in the study, they will be provided with further details and an informed consent form by one of the study members. The model consent form and other related documentation given to participants (all in German) can be obtained by the corresponding author upon request.

A separate list with patients screened, but who are not enrolled will contain information regarding the number of patients and the reasons for not enrolling. This list will be stored in the Trial Master File or Investigator Site File and is only accessible by the research team and the persons responsible for monitoring, audits, or inspections.

After the statistical analysis of this trial, the sponsor will make every effort to publish the data in a peer-reviewed medical journal, thereby adhering to the CONSORT reporting standards ${ }^{46}$ and SPIRIT guidelines. ${ }^{47}$ The use of professional writers is not foreseen. Authorship eligibility is defined according to the International Committee of Medical Journal Editors (ICMJE). Furthermore, presentations at congresses and reporting in a patient and healthcare professional magazine are planned.

\section{REFERENCES}

1. Murray CJ, Barber RM, Foreman KJ, Abbasoglu Ozgoren A, Abd-Allah F, Abera SF, Aboyans $\mathrm{V}$, et al. Global, regional, and national disability-adjusted life years (DALYs) for 306 diseases and injuries and healthy life expectancy (HALE) for 188 countries, 1990-2013: quantifying the epidemiological transition. Lancet. 2015;386(10009):2145-2191.

2. Feigin VL, Krishnamurthi RV, Parmar P, Norrving B, Mensah GA, Bennett DA, Barker-Collo S, et al. Update on the global burden of ischemic and hemorrhagic stroke in 1990-2013: The GBD 2013 Study. Neuroepidemiology. 2015;45(3):161-176.

3. Pang MY, Harris JE, Eng JJ. A community-based upper-extremity group exercise program improves motor function and performance of functional activities in chronic stroke: a randomized controlled trial. Archives of physical medicine and rehabilitation. 2006;87(1):1-9.

4. Langhorne P, Coupar F, Pollock A. Motor recovery after stroke: a systematic review. The Lancet. Neurology. 2009;8(8):741-754.

5. Jorgensen HS, Nakayama H, Raaschou HO, Vive-Larsen J, Stoier M, Olsen TS. Outcome and time course of recovery in stroke. Part II: Time course of recovery. The Copenhagen Stroke Study. Archives of physical medicine and rehabilitation. 1995;76(5):406-412.

6. Bernhardt J, Hayward KS, Kwakkel G, Ward NS, Wolf SL, Borschmann K, Krakauer JW, et al. Agreed definitions and a shared vision for new standards in stroke recovery research: The Stroke Recovery and Rehabilitation Roundtable taskforce. International journal of stroke: official journal of the International Stroke Society. 2017;12(5):444-450.

7. Taub E, Uswatte G, Mark VW, Morris DM. The learned nonuse phenomenon: implications for rehabilitation. Europa medicophysica. 2006;42(3):241-256.

8. Langhorne P, Bernhardt J, Kwakkel G. Stroke rehabilitation. Lancet. 2011;377(9778):1693-1702.

9. Lohse KR, Lang CE, Boyd LA. Is more better? Using metadata to explore dose-response relationships in stroke rehabilitation. Stroke; a journal of cerebral circulation. 2014;45(7):20532058 . 
10. Veerbeek JM, van Wegen E, van Peppen R, van der Wees PJ, Hendriks E, Rietberg M, Kwakkel G. What is the evidence for physical therapy poststroke? A systematic review and meta-analysis. PLoS One. 2014;9(2):e87987.

11. Subramanian SK, Massie CL, Malcolm MP, Levin MF. Does provision of extrinsic feedback result in improved motor learning in the upper limb poststroke? A systematic review of the evidence. Neurorehabilitation and neural repair. 2010;24(2):113-124.

12. Mehrholz J, Pohl M, Platz T, Kugler J, Elsner B. Electromechanical and robot-assisted arm training for improving activities of daily living, arm function, and arm muscle strength after stroke. The Cochrane database of systematic reviews. 2015(11):Cd006876.

13. Saposnik G, Cohen LG, Mamdani M, Pooyania S, Ploughman M, Cheung D, Shaw J, et al. Efficacy and safety of non-immersive virtual reality exercising in stroke rehabilitation (EVREST): a randomised, multicentre, single-blind, controlled trial. The Lancet. Neurology. 2016;15(10):1019-1027.

14. Noorkoiv M, Rodgers H, Price CI. Accelerometer measurement of upper extremity movement after stroke: a systematic review of clinical studies. Journal of neuroengineering and rehabilitation. 2014;11:144.

15. Gebruers N, Vanroy C, Truijen S, Engelborghs S, De Deyn PP. Monitoring of physical activity after stroke: a systematic review of accelerometry-based measures. Archives of physical medicine and rehabilitation. 2010;91(2):288-297.

16. Uswatte G, Giuliani C, Winstein C, Zeringue A, Hobbs L, Wolf SL. Validity of accelerometry for monitoring real-world arm activity in patients with subacute stroke: evidence from the extremity constraint-induced therapy evaluation trial. Archives of physical medicine and rehabilitation. 2006;87(10):1340-1345.

17. Uswatte G, Foo WL, Olmstead H, Lopez K, Holand A, Simms LB. Ambulatory monitoring of arm movement using accelerometry: an objective measure of upper-extremity rehabilitation in persons with chronic stroke. Archives of physical medicine and rehabilitation. 2005;86(7):14981501.

18. Markopoulos P, Timmermans AA, Beursgens L, van Donselaar R, Seelen HA. Us'em: the usercentered design of a device for motivating stroke patients to use their impaired arm-hand in daily life activities. IEEE Engineering in Medicine and Biology Society Annual Conference. 2011;2011:5182-5187.

19. Held JP, Klaassen B, Van Beijnum BF, Luft AR, Veltink PH. Usability evaluation of a vibrotactile feedback system in stroke subjects. Frontiers in bioengineering and biotechnology. 2017;4:98.

20. Winstein CJ. Knowledge of results and motor learning--implications for physical therapy. Physical therapy. 1991;71(2):140-149.

21. Van Vliet PM, Wulf G. Extrinsic feedback for motor learning after stroke: what is the evidence? Disability and rehabilitation. 2006;28(13-14):831-840.

22. Wang Q, Markopoulos P, Yu B, Chen W, Timmermans A. Interactive wearable systems for upper body rehabilitation: a systematic review. Journal of neuroengineering and rehabilitation. 2017;14(1):20.

23. Hoonhorst MH, Nijland RH, Van den Berg JS, Emmelot CH, Kollen BJ, Kwakkel G. How do Fugl-Meyer arm motor scores relate to dexterity according to the Action Research Arm Test at 6 months poststroke? Archives of physical medicine and rehabilitation. 2015;96(10):1845-1849.

24. Van der Lee JH, Beckerman H, Knol DL, De Vet HC, Bouter LM. Clinimetric properties of the motor activity log for the assessment of arm use in hemiparetic patients. Stroke. 2004;35(6):1410-1414.

25. Taub E, Miller NE, Novack TA, Cook EW, 3rd, Fleming WC, Nepomuceno CS, Connell JS, et al. Technique to improve chronic motor deficit after stroke. Archives of physical medicine and rehabilitation. 1993;74(4):347-354. 
26. Uswatte G, Taub E, Morris D, Vignolo M, McCulloch K. Reliability and validity of the upperextremity Motor Activity Log-14 for measuring real-world arm use. Stroke. 2005;36(11):24932496.

27. Fugl-Meyer AR, Jaasko L, Leyman I, Olsson S, Steglind S. The post-stroke hemiplegic patient. 1. a method for evaluation of physical performance. Scandinavian journal of rehabilitation medicine. 1975;7(1):13-31.

28. Gladstone DJ, Danells CJ, Black SE. The fugl-meyer assessment of motor recovery after stroke: a critical review of its measurement properties. Neurorehabilitation and neural repair. 2002;16(3):232-240.

29. Lyle RC. A performance test for assessment of upper limb function in physical rehabilitation treatment and research. International journal of rehabilitation research. Internationale Zeitschrift fur Rehabilitationsforschung. Revue internationale de recherches de readaptation. 1981;4(4):483-492.

30. Van der Lee JH, De Groot V, Beckerman H, Wagenaar RC, Lankhorst GJ, Bouter LM. The intra- and interrater reliability of the action research arm test: a practical test of upper extremity function in patients with stroke. Archives of physical medicine and rehabilitation. 2001;82(1):1419.

31. Yozbatiran N, Der-Yeghiaian L, Cramer SC. A standardized approach to performing the action research arm test. Neurorehabilitation and neural repair. 2008;22(1):78-90.

32. Herdman M, Gudex C, Lloyd A, Janssen M, Kind P, Parkin D, Bonsel G, et al. Development and preliminary testing of the new five-level version of EQ-5D (EQ-5D-5L). Quality of life research: an international journal of quality of life aspects of treatment, care and rehabilitation. 2011;20(10):1727-1736.

33. Golicki D, Niewada M, Karlinska A, Buczek J, Kobayashi A, Janssen MF, Pickard AS. Comparing responsiveness of the EQ-5D-5L, EQ-5D-3L and EQ VAS in stroke patients. Quality of life research: an international journal of quality of life aspects of treatment, care and rehabilitation. 2015;24(6):1555-1563.

34. Van Swieten JC, Koudstaal PJ, Visser MC, Schouten HJ, Van Gijn J. Interobserver agreement for the assessment of handicap in stroke patients. Stroke. 1988;19(5):604-607.

35. Farrell B, Godwin J, Richards S, Warlow C. The United Kingdom transient ischaemic attack (UK-TIA) aspirin trial: final results. Journal of neurology, neurosurgery, and psychiatry. 1991;54(12):1044-1054.

36. Wilson JT, Hareendran A, Grant M, Baird T, Schulz UG, Muir KW, Bone I. Improving the assessment of outcomes in stroke: use of a structured interview to assign grades on the modified Rankin Scale. Stroke. 2002;33(9):2243-2246.

37. Lang CE, Edwards DF, Birkenmeier RL, Dromerick AW. Estimating minimal clinically important differences of upper-extremity measures early after stroke. Archives of physical medicine and rehabilitation. 2008;89(9):1693-1700.

38. Oldfield RC. The assessment and analysis of handedness: the Edinburgh inventory. Neuropsychologia. 1971;9(1):97-113.

39. Bickerton WL, Samson D, Williamson J, Humphreys GW. Separating forms of neglect using the Apples Test: validation and functional prediction in chronic and acute stroke. Neuropsychology. 2011;25(5):567-580.

40. Brott T, Adams HP, Jr., Olinger CP, Marler JR, Barsan WG, Biller J, Spilker J, et al. Measurements of acute cerebral infarction: a clinical examination scale. Stroke. 1989;20(7):864-870.

41. Kelly PJ, Furie KL, Shafqat S, Rallis N, Chang Y, Stein J. Functional recovery following rehabilitation after hemorrhagic and ischemic stroke. Archives of physical medicine and rehabilitation. 2003;84(7):968-972. 
42. Schepers VP, Ketelaar M, Visser-Meily AJ, De Groot V, Twisk JW, Lindeman E. Functional recovery differs between ischaemic and haemorrhagic stroke patients. Journal of rehabilitation medicine. 2008;40(6):487-489.

43. Popovic MD, Kostic MD, Rodic SZ, Konstantinovic LM. Feedback-mediated upper extremities exercise: increasing patient motivation in poststroke rehabilitation. BioMed research international. 2014;2014:520374.

44. Kwakkel G, Veerbeek JM, Van Wegen EE, Wolf SL. Constraint-induced movement therapy after stroke. The Lancet. Neurology. 2015;14(2):224-234.

45. Duncan PW, Goldstein LB, Matchar D, Divine GW, Feussner J. Measurement of motor recovery after stroke. Outcome assessment and sample size requirements. Stroke. 1992;23(8):1084-1089.

46. Schulz KF, Altman DG, Moher D. CONSORT 2010 Statement: updated guidelines for reporting parallel group randomised trials. BMC medicine. 2010;8:18.

47. Chan AW, Tetzlaff JM, Gotzsche PC, Altman DG, Mann H, Berlin JA, Dickersin K, et al. SPIRIT 2013 explanation and elaboration: guidance for protocols of clinical trials. British Medical Journal (Clinical research ed.). 2013;346:e7586. 



\section{Chapter 5}

\section{Self-directed arm therapy at home after stroke with a sensor-based virtual reality training system}

F. Wittmann*, J.P. Held, O. Lambercy, M.L. Starkey, A. Curt, R. Hover, R. Gassert, A.R. Luft, R.R. Gonzenbach

* Both authors contributed equally. 


\section{ABSTRACT}

Background The effect of rehabilitative training after stroke is dose-dependent. Out-patient rehabilitation training is often limited by transport logistics, financial resources and a lack of motivation/compliance. We studied the feasibility of an unsupervised arm therapy for self-directed rehabilitation therapy in patients' homes.

Methods An open-label, single group study involving eleven patients with hemiparesis due to stroke ( $27 \pm 31.5$ months post-stroke) was conducted. The patients trained with an inertial measurement unit (IMU)-based virtual reality system (ArmeoSenso) in their homes for six weeks. The self-selected dose of training with ArmeoSenso was the principal outcome measure whereas the Fugl-Meyer Assessment of the upper extremity (FMA-UE), the Wolf Motor Function Test (WMFT) and IMU-derived kinematic metrics were used to assess arm function, training intensity and trunk movement. Repeated measures one-way ANOVAs were used to assess differences in training duration and clinical scores over time.

Results All subjects were able to use the system independently in their homes and no safety issues were reported. Patients trained on $26.5 \pm 11.5$ days out of 42 days for a duration of $137 \pm 120$ min per week. The weekly training duration did not change over the course of six weeks $(\mathrm{p}=0.146)$. The arm function of these patients improved significantly by 4.1 points ( $\mathrm{p}=0.003$ ) in the FMA-UE. Changes in the WMFT were not significant $(\mathrm{p}=0.552)$. ArmeoSenso based metrics showed an improvement in arm function, a high number of reaching movements (387 per session), and minimal compensatory movements of the trunk while training.

Conclusions Self-directed home therapy with an IMU-based home therapy system is safe and can provide a high dose of rehabilitative therapy. The assessments integrated into the system allow daily therapy monitoring, difficulty adaptation and detection of maladaptive motor patterns such as trunk movements during reaching.

Clinical trial registration URL: http://www.clinicaltrials.gov. Unique identifier: NCT02098135. 


\section{BACKGROUND}

Functional outcome following stroke is positively correlated with the dose of the applied rehabilitative intervention. ${ }^{1}$ Therefore, post-stroke therapy should be provided at a high intensity, a high frequency and over long periods of time. ${ }^{1,2}$ However, the delivery of intensive physical therapy requires extensive therapist support, is expensive, and is often limited by the low compliance and lack of motivation to perform rehabilitative training at the recommended frequency. ${ }^{3}$ This can lead to functional deterioration, e.g., by learned non-use of the affected limb. ${ }^{4}$

Self-directed home therapy, supported by dedicated instrumented devices ${ }^{5-7}$ or virtual reality gaming platforms, ${ }^{8-13}$ could help to increase the dose of rehabilitation at low cost without the need for direct supervision by a therapist. It is important that such home therapy adapts to changes in the subject's performance in order for it to remain challenging and motivating. ${ }^{8}$ On the other hand, unsupervised rehabilitative training could lead to inefficient or harmful (i.e. maladaptive) movement sequences or pain, and could potentially worsen performance. ${ }^{8,11,14}$ Home therapy should, therefore, include monitoring of movement quantity and quality. Several platforms dedicated to upper-extremity home rehabilitation have been proposed. ${ }^{6,715-17}$ However, to the best of our knowledge only few were actually installed in the patients' homes for several weeks and tested for feasibility beyond case studies. These home studies always involved some external supervision, in the form of e.g. on-site visits, ${ }^{16,17}$ tele-monitoring and adaption ${ }^{16,17}$ or telephone calls, ${ }^{6,7}$ which might have affected compliance and motivation and thereby therapy dosage. However, such an approach requires manpower, which limits the affordability and scalability of home-based therapy. The feasibility and compliance of completely unsupervised upper limb stroke therapy over the course of several weeks remains to be investigated. In this paper we investigate the feasibility of self-directed home training with the customdesigned ArmeoSenso system, ${ }^{18}$ a virtual reality arm rehabilitation platform based on wearable inertial measurement units (IMU). In a clinical study involving eleven patients with hemiparesis of the arm due to stroke, we evaluated the ability to deliver therapy at a high dose through simple-to-use and entertaining, yet functionally relevant and adaptive rehabilitation games. Unsupervised, automated assessments integrated into each therapy session allowed monitoring of arm function, and detection of undesired compensatory movements. 


\section{METHODS}

\section{ArmeoSenso training system}

ArmeoSenso comprises a motion capture system based on wearable sensors in combination with an all-in-one touch screen computer (Inspiron 2330, Dell Inc., Figure 5.1A). The therapy software provides a user-friendly graphical user interface, two therapy games, and two short automated assessments of arm function. ${ }^{18}$ For real-time tracking of arm and trunk movements, the patient wears three IMUs (MotionPod 3, Movea Inc.) fixed to the lower and upper arm as well as the trunk (Figure 5.1A). The IMUs measure acceleration, angular velocity and the magnetic field, all in three dimensions, and stream this data wirelessly to a receiver block, which is connected to the computer via USB and serves as a docking station to charge the sensors. A kinematic reconstruction estimates the orientation of the trunk, the upper- and the lower arm based on the Madgwick algorithm ${ }^{19}$ and the corresponding joint positions are computed with forward kinematics. ${ }^{20}$ This reconstruction serves as input for the assessments and therapeutic virtual reality games (Figure 5.1B). By using the same virtual kinematic parameters for each patient, virtual sizes, e.g. distances or the size of targets, are normalized to the patient's body size. To discourage trunk inclination or rotation during pointing movements, the arm movements are computed and displayed relative to the trunk.

\section{Sequence of a training session}

A typical training session is illustrated in Figure 5.1C. The patient uses the unaffected hand to touch a start button on the screen, which triggers visual instructions on how to remove the IMUs from the receiver block, don them on and perform a simple calibration procedure (i.e. guided start). For the calibration, the patient has to sit upright and hold the impaired arm in a horizontal position directed towards the screen for five seconds to determine the orientation of the IMUs on the patient's body. For calibration, the patient was allowed to use the unaffected arm for support.

Automated unsupervised assessments, conducted before every therapy session, evaluate arm function on two standardized tasks that remained identical throughout the therapy. The first was a pointing task which aims to evaluate the ability and time required for reaching a virtual target. The targets appear consecutively and in random order at nine pre-defined target positions located within the reachable workspace of a healthy individual. The patients are instructed to reach the target as quickly as possible and then stay on the target for two seconds. If a target is not reached within eight seconds, it disappears and a penalty time of eight seconds is taken instead. The number of targets reached and the mean time to reach 
A

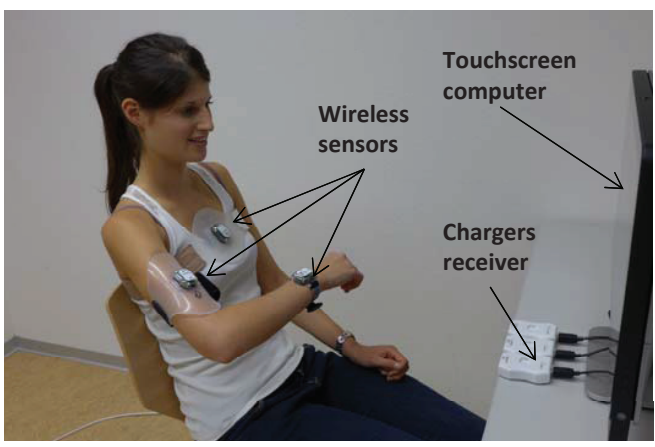

$\mathrm{B}$

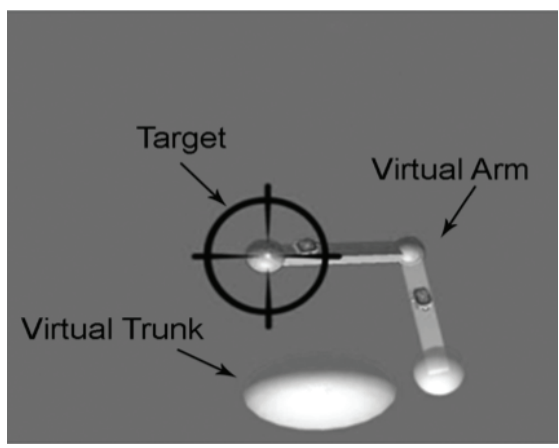

C

$D$
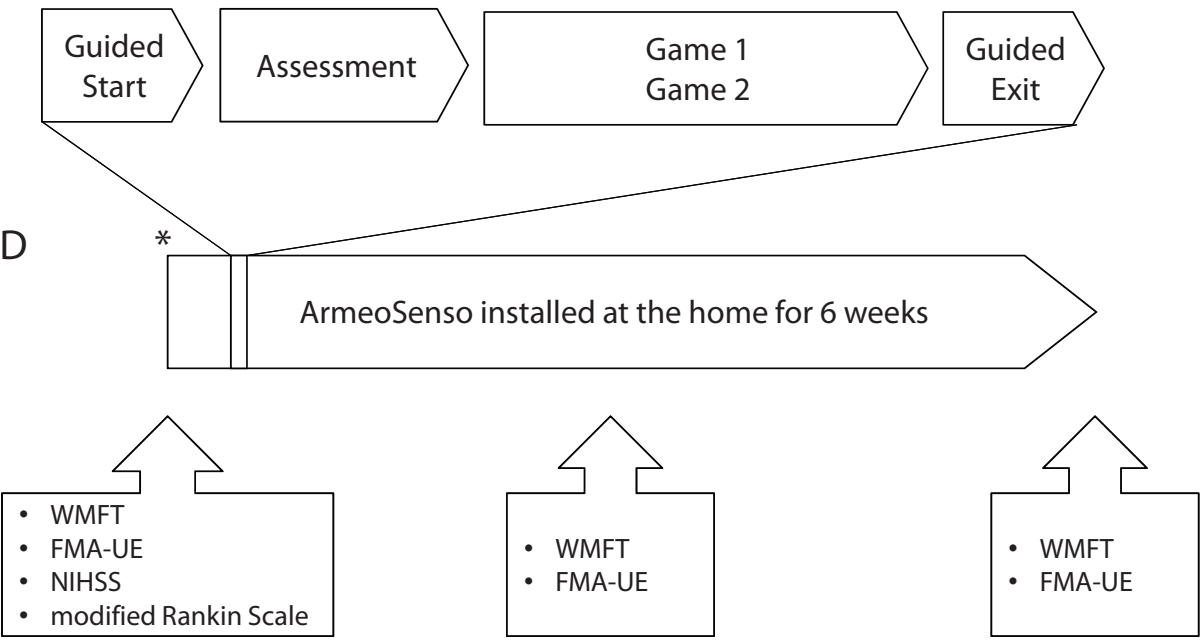

Figure 5.1: System overview and study outline.

A) Photograph of a healthy subject using ArmeoSenso. B) Screenshot of the pointing task assessment: the virtual upper- and lower arm and the trunk are displayed. The arm points to a target. C) Sequence of a training session. Before each training session, two automated assessments are performed. D) Study outline: The ArmeoSenso system is installed in the patient's home for six weeks. The patients are assessed clinically before the start, after three weeks, and after six weeks of training. Abbreviations: WMFT: Wolf Motor Function Test; FMA-UE: Fugl-Meyer Assessment Upper Extremity; NIHSS: National Institute of Health Stroke Scale. * System installation and patient instruction by a therapist.

the targets are reported. Joint angles are recorded to detect maladaptive compensatory movements such as excessive trunk inclination or trunk rotation during reaching.

The second assessment measured the two-dimensional workspace of the impaired arm in the transverse plane. Patients are instructed to actively reach out as far as possible with their 
impaired arm and to explore the entire arm workspace, similar to previous studies. ${ }^{21}$ The attained workspace is displayed and computed as the number of squares of ten centimeters side length arranged in a transverse plane relative to the patient' s trunk.

Therapy games: The aim of the therapy game 'Meteors', was to improve arm workspace and reaching velocity. In this game scenario, a virtual arm which matches the movement of the patient' $s$ arm is used to catch meteors that fall towards a planet. In contrast, the aim of the therapy game 'Slingshot' was to train arm coordination and to improve the precision of arm pointing and reaching movements. The patient holds a virtual slingshot to shoot stones at static or moving targets of variable size by pointing at the target with the slingshot and extending the elbow according to the target, which requires both precision and endurance. In both games, a performance-based (i.e. speed, number of targets reached, etc.) score is computed and used to dynamically adapt the difficulty of the game (e.g. meteors and targets move faster, or appear smaller etc.) in order to keep motivation and engagement high. The targets are placed within or at the border of the patient's 3D workspace, which is continuously estimated with a voxel-based model, to keep the challenge high, promote an increase in arm workspace, and prevent frustration. ${ }^{18}$

\section{Study design}

The study was designed as an open-label, single group clinical trial to study the feasibility and safety of performing arm rehabilitation with the ArmeoSenso system in the patient's home without any supervision. Inclusion criteria were a minimum age of 18 years, hemiparesis of the arm due to cerebrovascular ischemia, the ability to lift the paretic arm against gravity, a minimal arm workspace of $20 \mathrm{~cm}$ x $20 \mathrm{~cm}$ in the horizontal plane and absence of aphasia, depression, dementia and hemianopia. ArmeoSenso was installed on a table, and instructions for proper usage were given by a trained physiotherapist to the patient, prior to the start of the study. No modification of the patient' s house was required. Patients were asked to use the system as often as possible over a period of six weeks. They decided by themselves about the training duration and frequency and could start or stop a therapy session at any time. The patients' usual therapy continued and was not altered during the study. A structured patient interview was conducted at the end of the trial. The study followed GCP-guidelines and was approved by the local Cantonal ethics committee Zurich (KEK-ZH: 2013- 0182) and the Swissmedic (2013-MD- 0019). All subjects gave written informed consent in accordance with the declaration of Helsinki. 


\section{Outcome measures}

The primary safety outcome was any adverse event related to the system that occurred during the study period. The primary outcome of the study was the duration of training per week with ArmeoSenso. This was used as an indicator of therapy acceptance and feasibility of unsupervised therapy. As a measure of motivation, we investigated whether system usage changed over time. We report the average training duration for every training week, the training duration per session (equal to the minutes of playing games per ArmeoSenso session) and the training frequency (equal to the number of days of ArmeoSenso usage). The sum of both the number of meteors caught (Meteors game) and the number of targets hit (Slingshot game) was used as a measure of training intensity. To assess the efficiency of training, we quantified the training duration in relation to the overall time spent with the system, which includes the time for automated assessments and for system setup.

To investigate whether patients compensated for their arm impairment by moving their trunk, we analyzed trunk rotation and inclination during successful pointing movements in the pointing task assessment for one target. Trunk rotation and inclination were recorded at onset (initiation) of the movement and once the hand reached the target (final). The respective absolute difference between initial and final trunk orientation was treated as the patient's trunk compensation. The subtraction also serves to remove bias, e.g. due to sensor misalignment or magnetic field disturbances, ${ }^{22,23}$ while the short duration of $8 \mathrm{~s}$ or less should minimize effects of orientation drift, ${ }^{24}$ e.g. due to gyroscope bias. As a control, patients performed the same task with their unaffected arm 10 consecutive times within one session at the end of the home trial.

Arm function was assessed clinically using the Fugl-Meyer Assessment - Upper Extremity $(\text { FMA-UE) })^{25}$ and the Wolf Motor Function Test (WMFT) ${ }^{26}$ at 3 time points (see Figure 5.1D) and with ArmeoSenso-based automated assessments, as described above.

\section{Statistical methods}

Descriptive statistics are reported as mean \pm standard deviation of the mean, and where relevant with ( $\min , \max$ ). All outcomes were inspected for normal distribution using the Kolmogorov-Smirnov test, prior to selection of appropriate statistical tests. The two tailed Mann-Whitney test was used to compare the average weekly training duration in patients with severe impairment of arm function against patients with moderate to mild impairment. A one-way repeated measures ANOVA was used to assess differences over time in training duration, clinical scores and automated assessments, in case of normally distributed data. Otherwise, the non-parametric Friedman test was used. Correlation analysis was used to 
examine the relationship between clinical assessments and ArmeoSenso-based assessments. Results were considered significant at $\mathrm{p}<0.05$.

\section{Patient characteristics}

Eleven patients were recruited in the University Hospital Zurich (for details see Table 5.1). In parallel to the study, all patients except for one received physical therapy, on average 3.9 sessions/week, corresponding to approximately $155 \mathrm{~min} /$ week (estimated duration of 40 min per therapy session). Only one patient reported that he had no prior experience in using computers, and 8 out of 11 patients reported that they had never played computer games before.

Table 5.1: Baseline characteristics

\begin{tabular}{lc}
\hline & Mean \pm SD* \\
\hline $\mathrm{N}$ & 11 \\
Male & 5 \\
Right side affected & $3 / 8$ \\
Age, $y$ & $60 \pm 11.5$ \\
Months post stroke & $27 \pm 31.5$ \\
NIHSS $†$ & $3.3 \pm 1.2$ \\
mRS $\ddagger$ & $1.9 \pm 0.1$ \\
FMA-UE § & $35.1 \pm 19.9$ \\
WMFT || & $52 \pm 39$ \\
\hline
\end{tabular}

* Standard deviation.

† National Institutes of Health Stroke Scale (0-42 points).

₹ modified Rankin Scale (0-6 points).

§ Fugl-Meyer Assessment - Upper Extremity (0-66 points).

|| Wolf Motor Function Test (0-75 points).

\section{RESULTS}

\section{Safety and system usage}

All subjects were able to use the system without supervision at their homes and there were no patient-reported adverse events. On average, patients used the system on $26.5 \pm$ 11.5 days ( $\min 8, \max 41$ ) out of 42 days (Figure 5.2C), corresponding to 4.4 days with training per week. The average training duration per week was $137 \pm 120 \mathrm{~min}(\min 15$, $\max$ 357). The weekly training duration did not change over the course of six weeks (one-way repeated measures ANOVA: $\mathrm{p}=0.146, \mathrm{~F}=1.912$, Figure 5.2A). According to the patient 
interviews, 8/11 patients would have liked to continue training with the system and the perceived therapy efficacy was high, with $8 / 11$ patients stating that the trial improved their arm function. Further, 9/11 of patients found the system to be motivating. The two patients who replied negatively were also within the group of those $3 / 11$ patients not stating a desire to continue training with the system. Further, these three patients had a significantly (Wilcoxon rank-sum test, $\mathrm{p}=0.049)$ lower initial FMA-UE score $(16 \pm 7.8)$
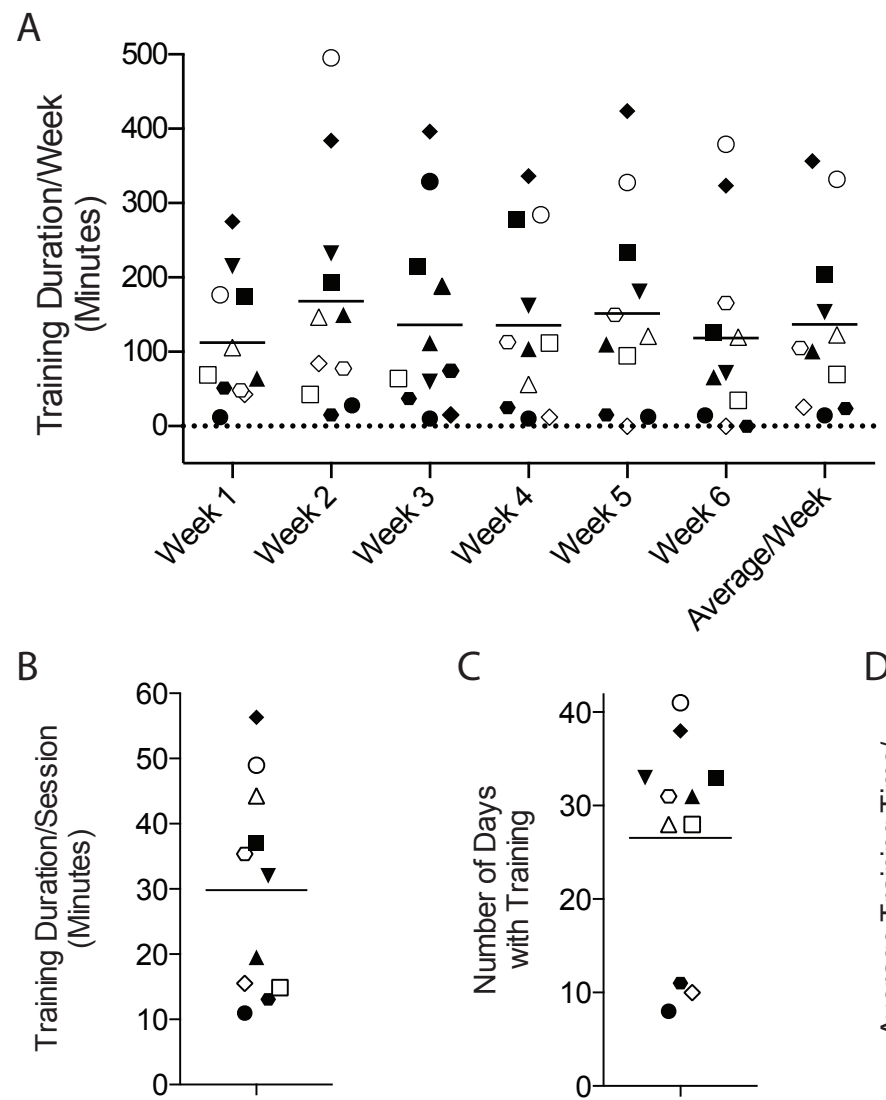

C

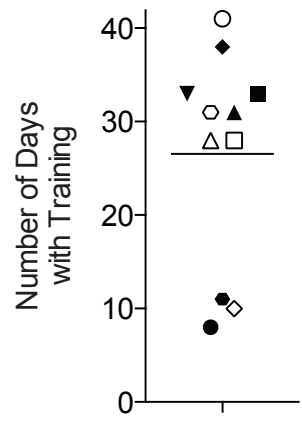

D
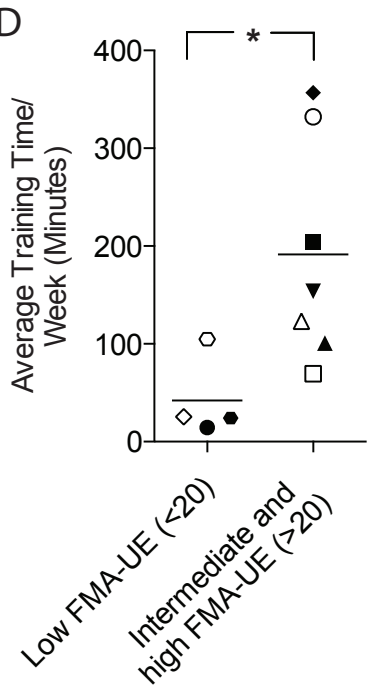

Figure 5.2: System usage.

A-D) Each symbol represents one patient. A) Weekly training duration for weeks 1-6 and average weekly training duration for each patient. B) Training duration per session. C) Number of days with training. Horizontal lines indicate averages. D) Average weekly training duration in patients with low ( $<20$ points) Fugl-Meyer Assessment Upper Extremity (FMA-UE) and intermediate to high ( $>20$ points) FMA-UE score. * Indicates significant differences in usage. 
compared to the other eight patients (FMA-UE $43 \pm 18.3$ ). These patients also trained less ( $85 \mathrm{~min} / \mathrm{w}$ versus $177 \mathrm{~min} / \mathrm{w}$ ), but the difference was not significant (Wilcoxon rank-sum test, $\mathrm{p}=0.38$ ).

The average training (gaming) duration per session was $30 \pm 16 \min (\min 11, \max 56$ ) (Figure 5.2B). The average number of successful arm movements during gaming with Meteors and Slingshot, a measure for training intensity, was $387 \pm 522$ movements per session (min 40, $\max 1486$ ). Patients with severe impairment of arm function (FMA-UE $\leq 20, \mathrm{~N}=4$ ) used ArmeoSenso significantly less (42 $\pm 42 \mathrm{~min} /$ week) than those with moderate and mild arm impairment (FMA-UE $>20, \mathrm{~N}=7,191 \pm 113 \mathrm{~min} /$ week, $\mathrm{p}=$ 0.024, Figure 5.2D).

The average setup duration per therapy session was $4 \pm 2 \mathrm{~min}$. The combined average time to complete all assessments for a therapy session was $4 \pm 1 \mathrm{~min}$. On average, patients spent $79 \%$ of a therapy session with actual training, i.e. playing either the Meteors or Slingshot therapy game.

\section{Changes in arm function}

Patients showed a significant improvement in the FMA-UE from $35.1 \pm 19.9$ points to 39.2 \pm 17.9 points after 6 weeks, which represents an average improvement of $4.1 \pm 2.5$ points (one way repeated measures ANOVA: $\mathrm{p}=0.003, \mathrm{~F}=8.701$, Figure 5.3A). The changes seen in the WMFT were small and not significant (improvement of +1.2 points after six weeks, Friedman-test: $\mathrm{p}=0.552$ ).

The automated assessments performed at each training session were plotted as twoweekly averages for comparison to clinical scores. The workspace of the affected arm in the transverse plane, as documented by the automated workspace assessment, improved significantly by $31 \%$ between the first two weeks ( $31.5 \pm 20.8$ squares) and the last two weeks ( $40.8 \pm 28$ squares; one way ANOVA: $\mathrm{p}=0.008, \mathrm{~F}=9.280$, Figure 5.3B). In the pointing task, the number of targets (out of 9 ) reached within $8 \mathrm{~s}$ improved significantly from $4.4 \pm$ 2.8 in the first two weeks to $5.9 \pm 3.1$ in the last two weeks (Friedman-test: $\mathrm{p}<0.001, \mathrm{~F}=$ 13.780, data not shown). The average time to reach the targets decreased significantly by $19 \%$, from $5.4 \pm 1.6 \mathrm{~s}$ in the first two weeks to $4.5 \pm 2.2 \mathrm{~s}$ in the last two weeks (one-way ANOVA: $\mathrm{p}=0.005, \mathrm{~F}=7.17$, Figure 5.3C).

The FMA-UE scores correlated significantly with all three metrics of the automated assessments (number of workspace voxels $\mathrm{r}=0.91, \mathrm{p}<0.001$, number of reached targets $\mathrm{r}=0.96, \mathrm{p}<0.001$, time to reach target $\mathrm{r}=0.92, \mathrm{p}<0.001$, the latter is shown in Figure 
5.3D). The therapy dose (i.e. total training duration with ArmeoSenso) did not correlate with the changes over six weeks in the clinical assessments $(r=-0.3, p=0.370$ for the FMA-UE, and $\mathrm{r}=-0.083, \mathrm{p}=0.809$ for the WMFT, data not shown).

\section{Kinematic analysis}

Trunk angle analysis during the pointing assessment shows that patients moved their trunk significantly more when reaching with their impaired arm compared to reaching with their

A

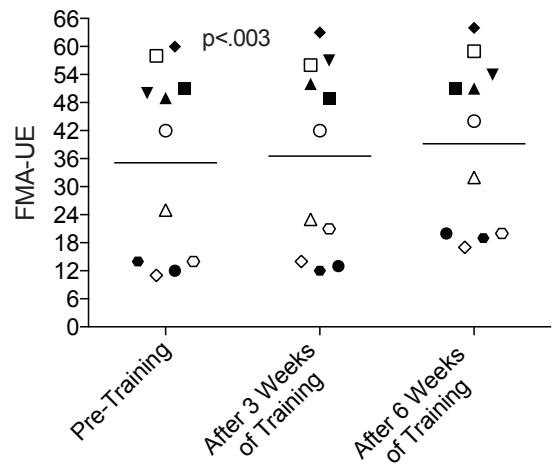

C

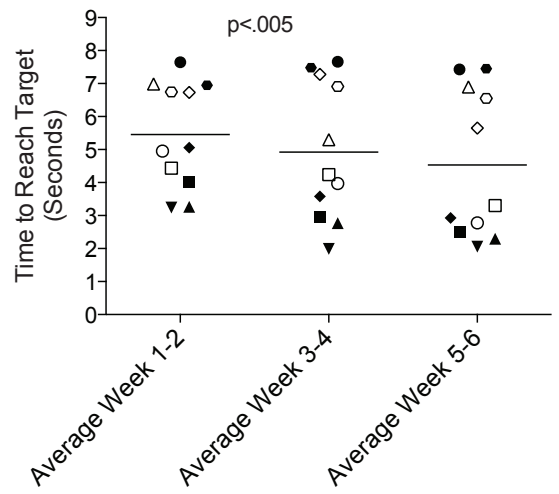

B

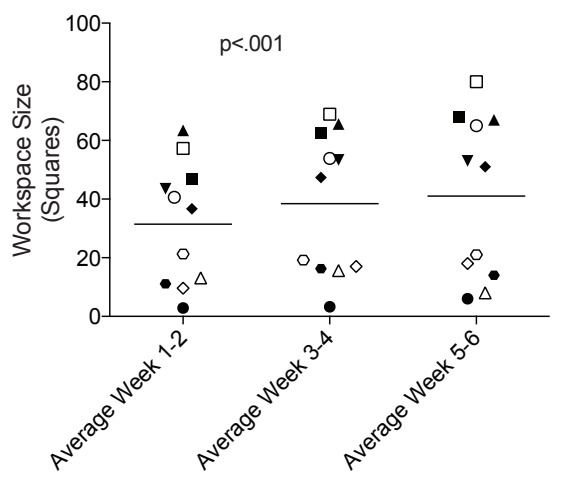

D

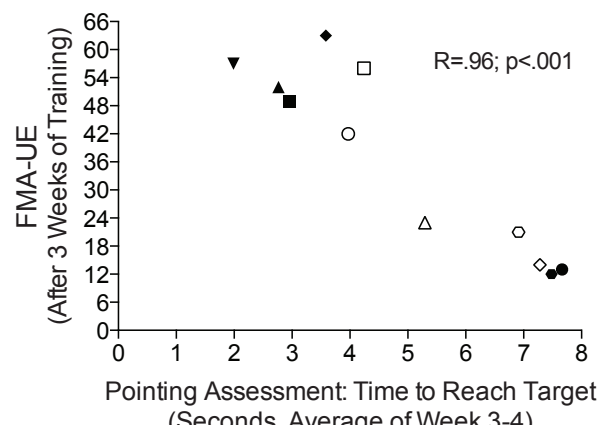

Figure 5.3: Arm function assessments.

A-D) Each symbol represents one patient. A-C) Horizontal bar = average. A) Fugl-Meyer Assessment Upper Extremity (FMA-UE) shows significant improvement after six weeks of therapy. B-D) ArmeoSenso-based Assessments. In one instance, a patient did not use the system during a block of two weeks. Here, the previous value was carried forward. B) Arm Workspace Assessment. The workspace is reported as squares, i.e. relative units for the covered workspace and shows significant improvement after six weeks. C) Pointing Task Assessment. The average time to reach target improves significantly. D) Significant correlation between clinical assessment (Fugl-Meyer assessment after 3 weeks of training) and ArmeoSenso assessment (time to reach target, average of training week 3-4, C). 
unaffected arm (Figure 5.4). These trunk movements occurred with a higher variability in the impaired side, as demonstrated by high standard deviations. The average absolute trunk rotation did not change significantly between the first and the last two weeks (oneway ANOVA, $\mathrm{p}=0.531, \mathrm{~F}=0.415$ ) but was significantly higher when compared to reaching movements with the unaffected arm (one-way ANOVA, $\mathrm{p}=0.030, \mathrm{~F}=5.859$ ). The same was observed for the average trunk inclination (for target 6), which did not change significantly between the first and the last two weeks (one-way ANOVA, $\mathrm{p}=0.208, \mathrm{~F}=1.757$ ) but was significantly higher compared to the sessions with the unaffected arm (one-way ANOVA, $\mathrm{p}$ $<0.001, F=24.968$ ). An example of the high inter-session variability of trunk angles during all training sessions for one patient (and the same target) is shown in Figures 5.4B and D.

A

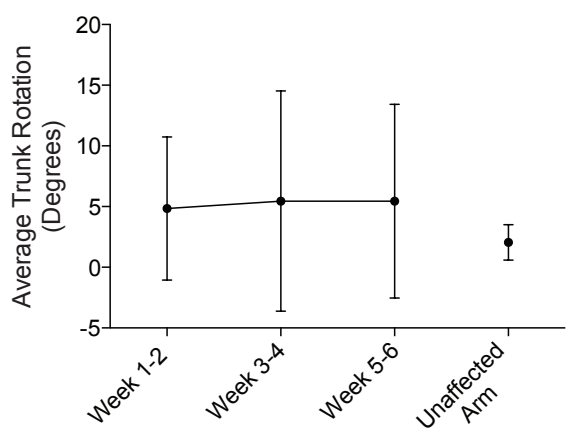

C

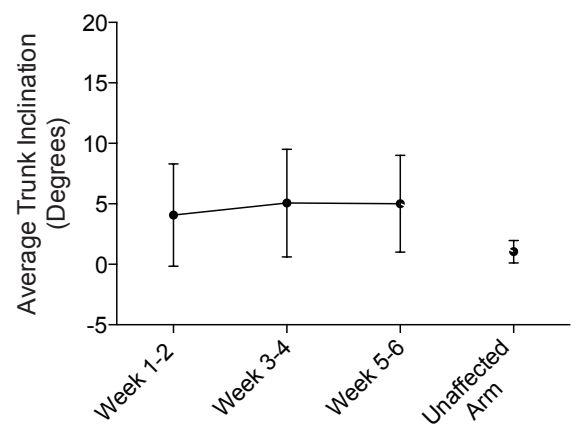

B

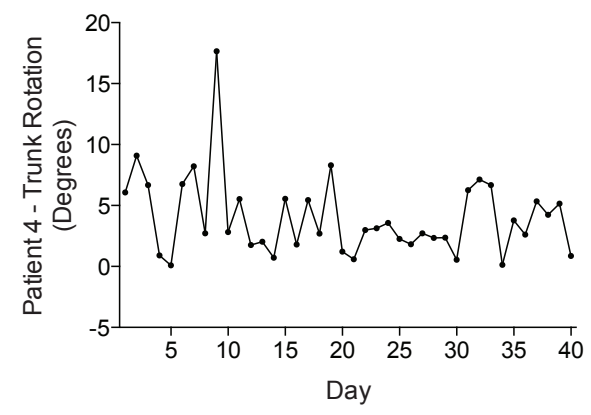

D

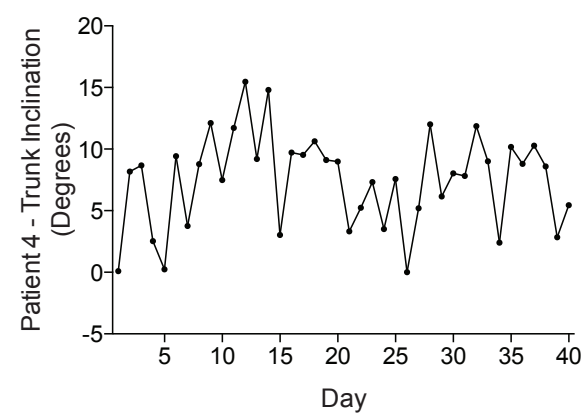

Figure 5.4: Trunk movement during pointing. Trunk rotation (A, B) and inclination (C, D) (two-weekly average) during pointing movements in the pointing task assessment for one specific target. For comparison, the values of 10 pointing movements performed with the unaffected limb are plotted $(N=8) . B+D)$ To demonstrate the high inter-session variability of trunk rotation and inclination during pointing movements, a complete dataset of one patient (impaired side) is plotted for the same target. Error bars: standard deviation. 


\section{DISCUSSION}

This paper presents results of a feasibility study using ArmeoSenso, a novel, wearable sensorbased home therapy system with rehabilitative games for arm training and automated IMUbased assessments of arm function. During a six-week intervention, all stroke patients ( $\mathrm{N}$ =11) were able to train with ArmeoSenso at home without therapist supervision and with no side effects reported, demonstrating that unsupervised selfdirected home therapy using a sensor-based virtual therapy platform is feasible and safe. As automated rehabilitation systems carry the risk of being unsuitable for stroke patients due to their complexity, we placed a high priority on developing a system that was easy to use, with therapy exercises that involved intuitive and meaningful, yet challenging movement tasks. ${ }^{27}$ The fact that elderly patients (6/11 were aged $>60$ years) and patients without gaming experience $(8 / 11)$ were able to successfully use the system supports its broad applicability. However, patients with severe impairments of arm function used the system less than those with moderate or mild impairments, suggesting that targeted training systems for this group should be developed, e.g. by addition of gravity support.

The therapy dose of $137 \mathrm{~min}$ per week (min/week) on average, with training sessions on 4.4 days per week, is promising. Despite the lack of any external therapy supervision after the initial setup day, this result compares favorably to other studies on unsupervised therapy in stroke, where doses of $105 \mathrm{~min} /$ week were achieved with the "Supervised Care \& Rehabilitation Involving Personal Telerobotics" (SCRIPT) hand orthosis, ${ }^{16,28} 85 \mathrm{~min} /$ week with the "home-based Computer Assisted Arm Rehabilitation" (hCAAR) actuated joystick ${ }^{7}$ or $31 \mathrm{~min} /$ week with the Virtual Glove upper limb rehabilitation system. ${ }^{17}$ Higher training doses of $214 \mathrm{~min} /$ week were achieved with the "Elinor" home therapy system, ${ }^{13}$ but mandatory weekly hospital visits might have influenced patient compliance. The average training intensity, which was 387 successful reaching and pointing movements per session, is in the range of another study with self-directed home therapy for subacute stroke patients, where 383 exercise repetitions per session were reported, ${ }^{29}$ and is much higher than the relatively low intensity typically observed in standard rehabilitation sessions for the upper limb (32 functional upper extremity movements per session ${ }^{30}$ ). The observed training duration did not decline during the six weeks intervention, indicating that the motivation to train with ArmeoSenso remained high. Overall system usage and the reported desire to continue training after completion of the study protocol suggest that the therapy could even be applied over longer periods. Training efficiency was also high with patients spending almost $80 \%$ of the time using the ArmeoSenso system with actual rehabilitation training. This compares favorably to training times in routine outpatient therapy. ${ }^{31}$ Such high training efficiency might lower the threshold to start a therapy session and thus 
increase the therapy dose within the available time. The system's permanent availability throughout the day in the subjects' home without the restriction of clinical schedules is an important advantage over tele-rehabilitation approaches. ${ }^{32,33}$ It would be interesting to know how many patients declined to participate and the reasons they give for this. However, this was not documented in this feasibility study. In two cases, there was insufficient space to set up the system in the patient' s homes, which illustrated the fact that size and footprint is an important design criterion for a home-based rehabilitation system.

In the unsupervised setting used here, research therapist did not see the patient over the course of the therapy. Direct monitoring of performance and progress and external intervention was therefore not possible. This motivated the development of short assessment modules that patients performed on a daily basis. To the best of our knowledge, unsupervised, automated assessments that accompany each training session have not been realized until now. The high correlation found between the automated assessments and clinical assessment scales in arm function is a first step towards confirming their validity. In the future, such unsupervised, automated assessments could alert therapists remotely, e.g. via the Internet, about stagnating or declining performance during home training sessions. Therapy games which do not take into account a patient's individual impairment, as with commercial entertainment systems designed for healthy users, are likely to frustrate patients, potentially jeopardizing motivation and compliance. ArmeoSenso therapy games constantly adapt their difficulty and intensity according to the subjects' performance, and place targets within or at the border of the reachable workspace 18 to maximize engagement and motivation of the subject.

An important function of a therapist is also to monitor and, if needed, correct the patient's posture and movements in order to prevent the development of pain or maladaptive motor patterns, such as excessive compensatory trunk movements (inclination and rotation) or excessive shoulder abduction during arm reaching. ${ }^{34}$ With systems that do not track joint angles (e.g. Nintendo Wii) or commercial games that are not designed for rehabilitation purposes, ${ }^{11,35}$ development of such patterns may go unnoticed. Systems based on the use of cameras (e.g. Kinect ${ }^{12,35-37}$ ) or IMUs that reconstruct body posture offer the possibility to detect compensatory movements. ${ }^{38}$ The reconstruction algorithm implemented in ArmeoSenso attempted to minimize trunk inclination and rotation by directly suppressing their effects in the virtual environment, i.e. only arm movements relative to the trunk are depicted and used as input for the games and assessments. Despite this effort, patients typically exhibited significantly higher trunk inclination and trunk rotation during reaching movements with their impaired arm than with the unimpaired arm. ${ }^{39}$ Nevertheless, trunk movements remained small in most patients, with an average of less than five degrees 
of trunk inclination or rotation. The extent of trunk movement was highly variable (interpatient and inter-session), and there was no significant trend over time that would suggest either an increase or a reduction of compensation with the trunk during reaching movements. Providing auditory instructions when excessive trunk movements are detected, simulating the presence of a virtual therapist, ${ }^{38}$ or using negative visual cues within the therapy game ${ }^{40}$ might help to prevent compensatory trunk movements.

The mean gain in FMA-UE was 4.1 points, which is not regarded as clinically relevant, but five out of eleven patients showed a clinically relevant improvement of more than 4.25 points. ${ }^{41}$ This is comparable to findings for high-intensity therapy in chronic stroke patients. ${ }^{42}$ This improvement was not reflected in the WMFT, which improved marginally by 1.2 points. This is likely due to the lack of hand training by ArmeoSenso; hand function is important for performing the WMFT. The improvement of arm function could be explained by the self-directed training with ArmeoSenso or by the standard rehabilitation therapy that most patients received during the study in addition to the experimental training (155 min per week on average).

\section{CONCLUSION}

This paper presents the design and feasibility of ArmeoSenso, a wearable sensor-based home therapy system for self-directed rehabilitative arm training after stroke. Our results demonstrate that this home therapy is safe and can provide rehabilitative training in a high dose. The integrated assessments allow daily therapy monitoring, difficulty adaptation and detection of maladaptive motor patterns such as trunk movements during reaching. Clinical effectiveness of ArmeoSenso needs to be investigated in a larger randomized controlled trial.

\section{Acknowledgements}

The authors would like to thank Mark van Raai, Dr. Kei Hoshi, Dr. Peter Schenk, Dr. Melissa Lawrence and Dr. Kaspar Leuenberger for their help in the implementation of the ArmeoSenso software, Irene Christen, Lydia Fischer, Werner Popp and Urs Albisser for their support with the study, Dr. Corinne Nicoletti for setup pictures, as well as all patients who participated in the study. 


\section{REFERENCES}

1. Kwakkel G. Impact of intensity of practice after stroke: issues for consideration. Disability and Rehabilitation. 2006;28(13-14):823-830.

2. Lohse KR, Lang CE, Boyd LA. Is more better? Using metadata to explore dose-response relationships in stroke rehabilitation. Stroke. 2014;45(7):2053-2058.

3. Lenze EJ, Munin MC, Quear T, Dew MA, Rogers JC, Begley AE, Reynolds CF. Significance of poor patient participation in physical and occupational therapy for functional outcome and length of stay. Archives of Physical Medicine and Rehabilitation. 2004;85(10):1599-1601.

4. Taub E. Movement in nonhuman primates deprived of somatosensory feedback. Exercise and Sport Sciences Reviews. 1976;4:335-374.

5. Kohler F, Schmitz-Rode T, Disselhorst-Klug C. Introducing a feedback training system for guided home rehabilitation. Journal of NeuroEngineering and Rehabilitation. 2010;7:2.

6. Zondervan DK, Augsburger R, Bodenhoefer B, Friedman N, Reinkensmeyer DJ, Cramer SC. Machine-based, self-guided home therapy for individuals with severe arm impairment after stroke: A randomized controlled trial. Neurorehabil Neural Repair. 2015;29(5):395-406.

7. Sivan M, Gallagher J, Makower S, Keeling D, Bhakta B, O’Connor RJ, Levesley M. Home-based Computer Assisted Arm Rehabilitation (hCAAR) robotic device for upper limb exercise after stroke: results of a feasibility study in home setting. Journal of NeuroEngineering and Rehabilitation. 2014;11(1):163.

8. Borghese NA, Pirovano M, Lanzi PL, Wuest S, de Bruin ED. Computational intelligence and game design for effective at-home stroke rehabilitation. Games for Health Journal. 2013;2(2):8188 .

9. Cameirao MDS, Badia SBI, Duarte E, Verschure PFMJ. Virtual reality based rehabilitation speeds up functional recovery of the upper extremities after stroke: A randomized controlled pilot study in the acute phase of stroke using the Rehabilitation Gaming System. Restorative Neurology and Neuroscience. 2011;29(5):287-298.

10. Saposnik G, Robert T, Mamdani M, Cheung D, Thorpe KE, McIlroy B, Willems J, et al. Effectiveness of Virtual Reality using Wii Gaming technology in STroke Rehabilitation (EVREST): a randomized clinical trial and proof of principle. Stroke. 2010;41(7):E473-E473.

11. Prosperini L, Fortuna D, Gianni C, Leonardi L, Marchetti MR, Pozzilli C. Home-based balance training using the Wii balance board: a randomized, crossover pilot study in multiple sclerosis. Neurorehabil Neural Repair. 2013;27(6):516-525.

12. Webster D, Celik O. Systematic review of Kinect applications in elderly care and stroke rehabilitation. Journal of NeuroEngineering and Rehabilitation. 2014;11:108.

13. Slijper A, Svensson KE, Backlund P, Engstrom H, Sunnerhagen KS. Computer game-based upper extremity training in the home environment in stroke persons: a single subject design. Journal of NeuroEngineering and Rehabilitation. 2014;11:35.

14. Liebermann DG, Berman S, Weiss PL, Levin MF. Kinematics of Reaching Movements in a 2-D Virtual Environment in Adults With and Without Stroke. IEEE Transactions on Neural Systems and Rehabilitation Engineering. 2012;20(6):778-787.

15. Kim GJ, Rivera L, Stein J. Combined Clinic-Home Approach for Upper Limb Robotic Therapy After Stroke: A Pilot Study. Archives of Physical Medicine and Rehabilitation. 2015;96(12):22432248.

16. Nijenhuis SM, Prange GB, Amirabdollahian F, Sale P, Infarinato F, Nasr N, Mountain G, et al. Feasibility study into self-administered training at home using an arm and hand device with motivational gaming environment in chronic stroke. Journal of Neuroengineering and Rehabilitation. 2015;12. 
17. Standen PJ, Threapleton K, Connell L, Richardson A, Brown DJ, Battersby S, Sutton CJ, et al. Patients' use of a home-based virtual reality system to provide rehabilitation of the upper limb following stroke. Physical Therapy. 2015;95(3):350-359.

18. Wittmann F, Lambercy O, Gonzenbach RR, van Raai MA, Hover R, Held J, Starkey ML, et al. Assessment-driven arm therapy at home using an IMU-based virtual reality system. Paper presented at: Rehabilitation Robotics (ICORR), 2015 IEEE International Conference on 2015.

19. Madgwick SOH, Harrison AJL, Vaidyanathan R. Estimation of IMU and MARG orientation using a gradient descent algorithm. 2011 Ieee International Conference on Rehabilitation Robotics (Icorr). 2011.

20. Craig JJ. Introduction to Robotics: Mechanics and Control. Pearson/Prentice Hall; 2005.

21. Sukal TM, Ellis MD, Dewald JP. Shoulder abduction-induced reductions in reaching work area following hemiparetic stroke: neuroscientific implications. Experimental Brain Research. 2007;183(2):215-223.

22. Roetenberg D, Luinge HJ, Baten CT, Veltink PH. Compensation of magnetic disturbances improves inertial and magnetic sensing of human body segment orientation. IEEE Transactions on Neural Systems and Rehabilitation Engineering. 2005;13(3):395-405.

23. Roetenberg D, Baten CT, Veltink PH. Estimating body segment orientation by applying inertial and magnetic sensing near ferromagnetic materials. IEEE Transactions on Neural Systems and Rehabilitation Engineering. 2007;15(3):469-471.

24. LaValle SM, Yershova A, Katsev M, Antonov M. Head tracking for the Oculus Rift. Paper presented at: 2014 IEEE International Conference on Robotics and Automation (ICRA); May 31 2014-June 72014.

25. Fugl-Meyer AR, Jaasko L, Leyman I, Olsson S, Steglind S. The post-stroke hemiplegic patient. 1. a method for evaluation of physical performance. Scandinavian Journal of Rehabilitation Medicine. 1975;7(1):13-31.

26. Wolf SL, Catlin PA, Ellis M, Archer AL, Morgan B, Piacentino A. Assessing Wolf motor function test as outcome measure for research in patients after stroke. Stroke. 2001;32(7):1635-1639.

27. Burke JW, McNeill MDJ, Charles DK, Morrow PJ, Crosbie JH, McDonough SM. Optimising engagement for stroke rehabilitation using serious games. Visual Computer. 2009;25(12):10851099 .

28. Amirabdollahian F, Ates S, Basteris A, Cesario A, Buurke J, Hermens H, Hofs D, et al. Design, development and deployment of a hand/wrist exoskeleton for home-based rehabilitation after stroke-SCRIPT project. Robotica. 2014;32(08):1331-1346.

29. Zondervan DK, Augsburger R, Bodenhoefer B, Friedman N, Reinkensmeyer DJ, Cramer SC. Machine-based, self-guided home therapy for individuals with severe arm impairment after stroke: A randomized controlled trial. Neurorehabil Neural Repair. 2014.

30. Lang CE, Macdonald JR, Reisman DS, Boyd L, Jacobson Kimberley T, Schindler-Ivens SM, Hornby TG, et al. Observation of amounts of movement practice provided during stroke rehabilitation. Archives of Physical Medicine and Rehabilitation. 2009;90(10):1692-1698.

31. De Wit L, Putman K, Dejaeger E, Baert I, Berman P, Bogaerts K, Brinkmann N, et al. Use of time by stroke patients: a comparison of four European rehabilitation centers. Stroke. 2005;36(9):1977-1983.

32. Laver KE, Schoene D, Crotty M, George S, Lannin NA, Sherrington C. Telerehabilitation services for stroke. Cochrane Database of Systematic Reviews. 2013;12:CD010255.

33. Johansson T, Wild C. Telerehabilitation in stroke care--a systematic review. Journal of Telemedicine and Telecare. 2011;17(1):1-6.

34. Cirstea MC, Levin MF. Compensatory strategies for reaching in stroke. Brain. 2000;123 ( Pt 5):940-953.

35. Sin $\mathrm{H}$, Lee G. Additional virtual reality training using Xbox Kinect in stroke survivors with hemiplegia. American Journal of Physical Medicine and Rehabilitation. 2013;92(10):871-880. 
36. Gama AD, Fallavollita P, Teichrieb V, Navab N. Motor rehabilitation using kinect: A systematic review. Games for Health Journal. 2015;4(2).

37. Taati B, Wang R, Huq R, Snoek J, Mihailidis A. Vision-based posture assessment to detect and categorize compensation during robotic rehabilitation therapy. 2012 4th Ieee Ras \& Embs International Conference on Biomedical Robotics and Biomechatronics (Biorob). 2012:1607-1613.

38. Johnson MJ, Shakya Y, Strachota E, Ahamed SI. Low-cost monitoring of patients during unsupervised robot/computer assisted motivating stroke rehabilitation. Biomedizinische Technik. 2011;56(1):5-9.

39. Mandon L, Boudarham J, Robertson J, Bensmail D, Roche N, Roby-Brami A. Faster reaching in chronic spastic stroke patients comes at the expense of arm-trunk coordination. Neurorehabilitation and neural repair. 2015:1545968315591704.

40. Alankus G, Kelleher C. Reducing Compensatory Motions in Motion-Based Video Games for Stroke Rehabilitation. Human-Computer Interaction. 2015;30(3-4):232-262.

41. Page SJ, Fulk GD, Boyne P. Clinically important differences for the upper-extremity FuglMeyer Scale in people with minimal to moderate impairment due to chronic stroke. Physical Therapy. 2012;92(6):791-798.

42. Wu X, Guarino P, Lo AC, Peduzzi P, Wininger M. Long-term effectiveness of intensive therapy in chronic stroke. Neurorehabilitation and Neural Repair. 2015:1545968315608448. 



\section{Chapter 6}

\section{Does motivation matter in upper limb rehabilitation after stroke? ArmeoSenso-Reward: Study protocol for a randomized controlled trial.}

M. Widmer ${ }^{\star}$, J.P. Held ${ }^{\star}$, F. Wittmann, O. Lambercy,

K. Lutz, A.R. Luft

* Both authors contributed equally. 


\section{ABSTRACT}

Background Fifty percent of all stroke survivors remain with functional impairments of their upper limb. While there is a need to improve the effectiveness of rehabilitative training, so far no new training approach has proven to be clearly superior to conventional therapy. As training with rewarding feedback has been shown to improve motor learning in humans, it is hypothesized that rehabilitative arm training could be enhanced by rewarding feedback. In this paper, we propose a trial protocol investigating rewards in the form of performance feedback and monetary gains as ways to improve effectiveness of rehabilitative training.

Methods This multicentric, assessor-blinded, randomized controlled trial uses the ArmeoSenso virtual reality rehabilitation system to train 74 first-ever stroke patients ( $<100$ days post stroke) to lift their impaired upper limb against gravity and to improve the workspace of the paretic arm. Three sensors are attached to forearm, upper arm, and trunk to track arm movements in three-dimensional space while controlling for trunk compensation. Whole-arm movements serve as input for a therapy game. The reward group $(n=37)$ will train with performance feedback and contingent monetary reward. The control group $(n=37)$ uses the same system but without monetary reward and with reduced performance feedback. Primary outcome is the change in the hand workspace in the transversal plane. Standard clinical assessments are used as secondary outcome measures.

Discussion This randomized controlled trial will be the first to directly evaluate the effect of rewarding feedback, including monetary rewards, on the recovery process of the upper limb following stroke. This could pave the way for novel types of interventions with significantly improved treatment benefits, e.g., for conditions that impair reward processing (stroke, Parkinson's disease).

Trial registration Trial registry number on cliniclatrials.gov is NCT02257125, first registered on 30 September 2014. 


\section{BACKGROUND}

After stroke, $50 \%$ of survivors are left with impairments in arm function, ${ }^{1,2}$ which is associated with reduced health-related quality of life. ${ }^{3}$ While there is evidence for a positive correlation between therapy dose and functional recovery, ${ }^{4-6}$ a higher therapy dose is challenging to implement, as it usually leads to an increase in costs commonly not covered by health insurances. However, when dose is matched, most randomized controlled trials introducing new types of rehabilitative interventions (e.g., robot-assisted therapy ${ }^{7}$ ) failed to show a superior effect compared to standard therapy. Thus, the need for improving therapy effectiveness remains. In search for elements of effective therapy, we hypothesize that performance feedback and monetary rewards can improve effectiveness.

It has been shown that reward enhances procedural ${ }^{8}$ and motor skill learning ${ }^{9,10}$ and has a positive effect on motor adaption. ${ }^{11}$ Rewards mainly improve retention of motor-skills and motor adaptions. ${ }^{9-11}$ This effect was not explained by training duration (dose) as rewarded and non-rewarded groups underwent similar training schedules. ${ }^{8-11}$ In a functional magnetic resonance imaging (fMRI) study, Widmer et al. reported that adding monetary rewards after good performance lead to better consolidation and higher ventral striatum activation than knowledge of performance alone. ${ }^{10}$ The striatum is a key focus of reward processing, ${ }^{12}$ and its activity was shown to be increased by both intrinsic and extrinsic reward. ${ }^{13}$ Being a brain structure that receives substantial dopaminergic input from the midbrain, ventral striatal activity can be seen as a surrogate marker for dopaminergic activity in substantia nigra/ventral tegmental area. ${ }^{14}$ In rodents, Hosp et al. found that dopaminergic projections from the midbrain also terminate directly in the primary motor cortex (M1). ${ }^{15}$ Dopamine in M1 is necessary for long-term potentiation of certain cortico-cortical connections and successful motor skill-learning. ${ }^{16}$ As mechanisms of motor learning are also thought to play a role in motor recovery, ${ }^{17}$ rehabilitative interventions may benefit from neuroplasticity enhanced by reward.

Here, we describe a trial protocol to test the effect of enhanced feedback and reward on arm rehabilitation after stroke at matched training dose (time and intensity). We use the ArmeoSenso, a standardized virtual reality (VR)-based training system ${ }^{18}$ that is delivered in two versions for two different study groups, one version with and one without reward and enhanced performance feedback. 


\section{METHODS}

\section{Ethics and reporting}

The study protocol follows the Consolidated Standards of Reporting Trials (CONSORT) Statement on randomised trials of non-pharmacological treatment ${ }^{19}$ and Standard Protocol Items: Recommendations for Interventional Trials (SPIRIT; see Figure 6.5 for the SPIRIT Figure and the SPIRIT Checklist) guidance for protocol reporting. ${ }^{20}$ The study is recruiting patients at three different rehabilitation clinics. The procedures and the protocol (version 4.1 of 18 August 2016) have been approved by the responsible Ethics Committees "Ethikkommission Nordwest- und Zentralschweiz" and the "Kantonale Ethikkommission Zürich" (LU2013-079 and PB_2016-01804), and the Swiss Agency for Therapeutic Products (Swissmedic: 2014-MD-0033) and conform to the guidelines of Good Clinical Practice E6 (R1). All subjects have to give written informed consent in accordance with the Declaration of Helsinki. A quality assurance audit/inspection of this study may be conducted by the competent authority or Ethical Committees, respectively. The quality assurance auditor/ inspector will have access to all medical records, the investigator's study related files and correspondence, and the informed consent documentation that is relevant to this clinical study.

\section{Study design}

This multicentric trial is randomized, controlled and assessor-blinded (Figure 6.1). Patients are unaware of the training characteristics of the other study group.

\section{Study population}

This study includes stroke patients (maximum 100 days after stroke) who meet the following criteria: minimum age of 18 years, hemiparesis of the arm due to cerebrovascular ischemia, the ability to lift the paretic arm against gravity, a minimal arm workspace of $20 \mathrm{~cm} \times 20$ $\mathrm{cm}$ in the horizontal plane, ability and willingness to participate, as well as the absence of severe aphasia (i.e., patients who are not able to follow two-stage commands), depression, dementia and hemianopia.

\section{Randomization}

The randomization procedure was planned and set up by an independent contract research organization (Appletree CI Group, Winterthur, Switzerland). A non-consecutively increasing, pseudo-randomly generated list of subject identification numbers (IDs) was 


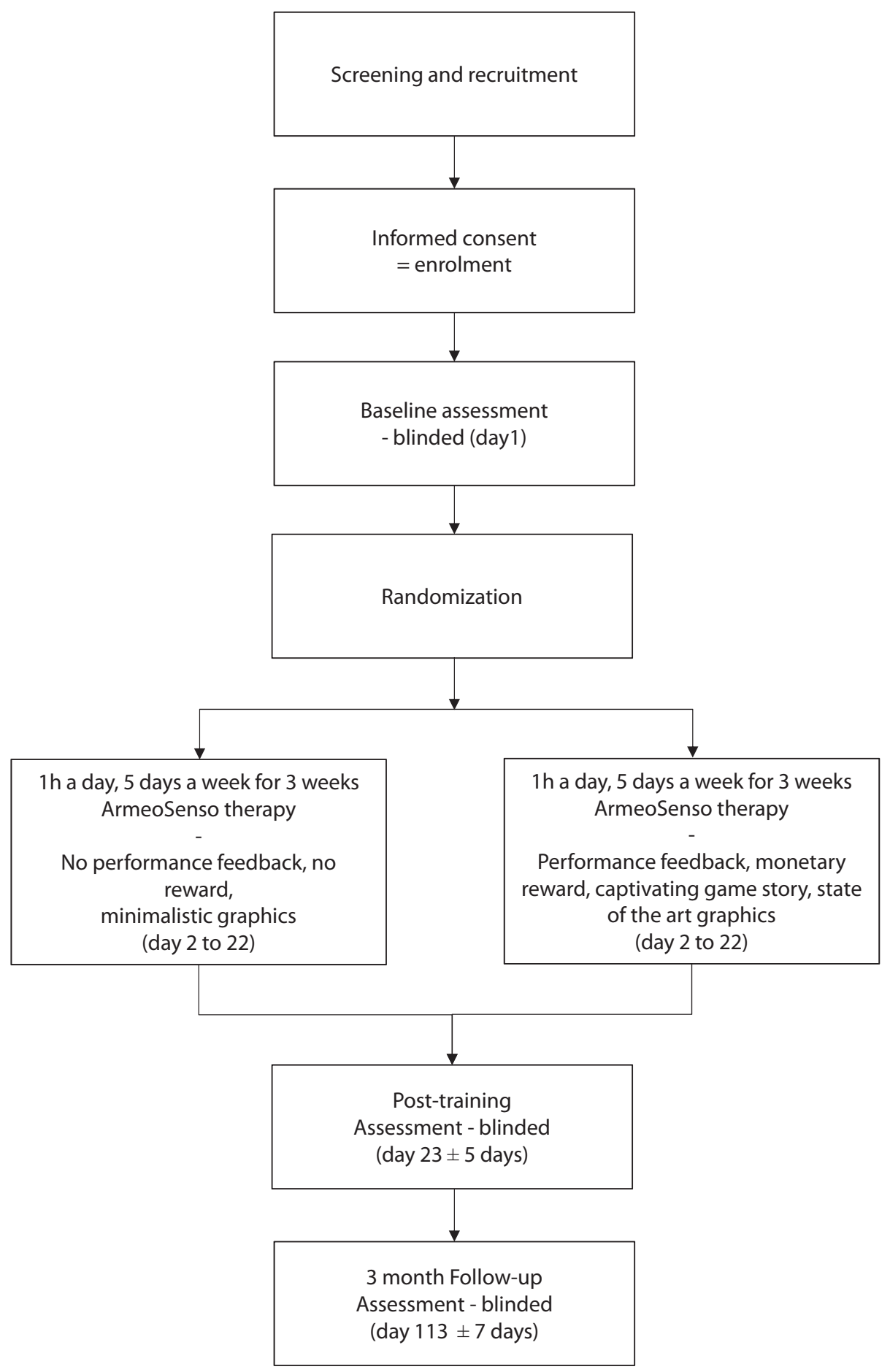

Figure 6.1: Flow diagram illustrating the trial design and sequence. 
created. IDs are chronologically assigned to each new study participant, stratified by the study center. Allocation to one of the two study groups is balanced in blocks of four. The randomization list containing the subject-ID, the corresponding group allocation and a randomly generated password was sent to an independent (unblinded) study staff member ("admin") who has set up respective patient-user computer accounts used for accessing the therapy game. The group-specific version of the game, i.e., either with or without reward, is defined by the account. The admin keeps the assignment list and is not involved in data collection.

Immediately before the first training session, each study participant has to confirm by signature to have received a sealed envelope containing a butterfly etiquette with ID and password to access the account. The patient keeps this etiquette for the entire study duration.

\section{ArmeoSenso Training System}

The arm rehabilitation system combines motion capturing via wearable inertial measurement units (IMUs) in combination with a therapy game, running on a touch screen computer (Inspiron 2330, Dell Inc., Round Rock, TX USA) (Figure 6.2A). Three wireless IMUs (MotionPod 3, Movea SA, Grenoble, France) are fixed to the functionally impaired lower and upper arm as well as the trunk. ${ }^{18,21}$

In contrast to robot-based VR therapy systems, this sensor-based approach does not offer any weight support for the impaired arm. The ArmeoSenso system specifically requires the patient to lift the arm against gravity and to increase hand workspace in three-dimensional (3D) space. The system was validated in a home feasibility trial with stroke patients. ${ }^{21}$ For the present study, the ArmeoSenso system includes two automated functional assessments, one consisting of a pointing task with nine targets arranged in two semicircles in the transversal
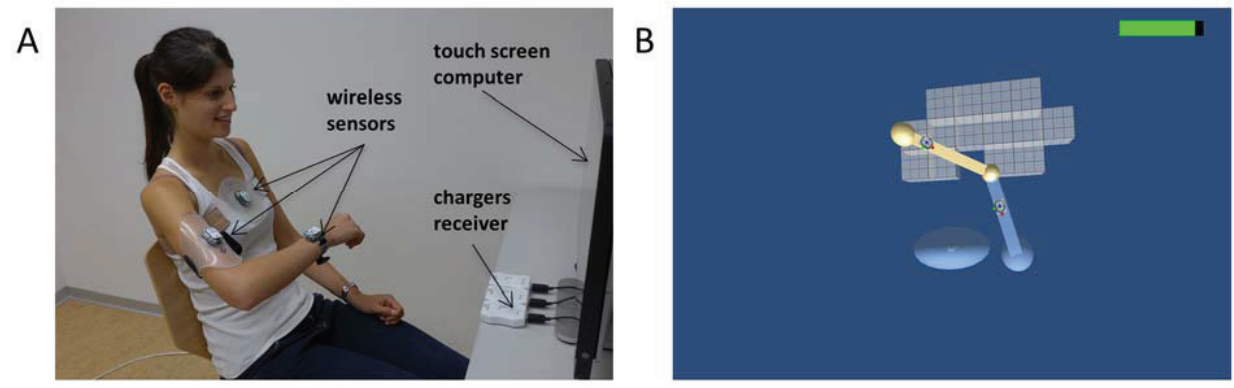

Figure 6.2: A) Healthy subject using the ArmeoSenso training system. B) Arm workspace assessment:grey cubic voxels arranged in the transverse plane reflecting $10 \mathrm{~cm} \times 10 \mathrm{~cm}$ active workspace relative to the patient's trunk. 

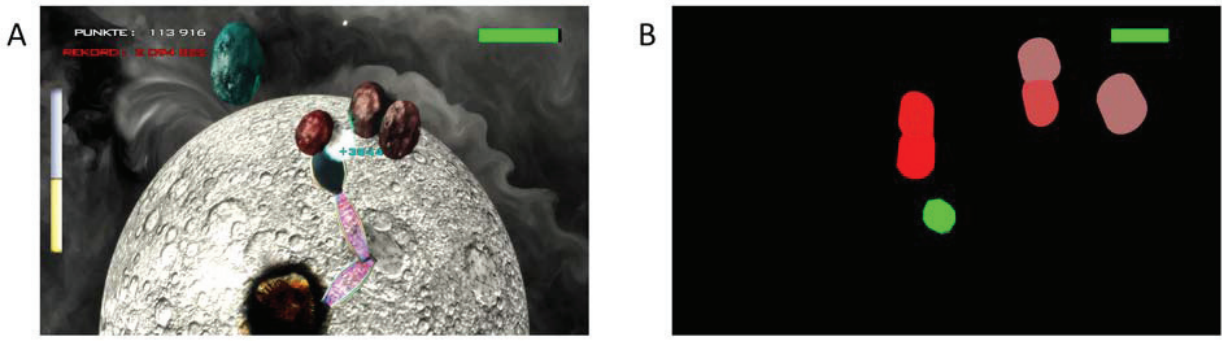

Figure 6.3: A) ArmeoSenso-Reward: METEORS therapy game. The hand of the virtual arm is used to catch the falling meteors before they crash onto the planet. If caught, the meteor explodes and a score appears. If missed, the planet gets damaged (note the impact crater). The current score (=PUNKTE) is displayed on the upper left (white font colour) and compared to the patient's all-time record (=REKORD; red font colour, upper left). The green bar on the upper right indicates resting time. If completely black, the patient has to rest for $4 \mathrm{~s}$ before new meteors are spawned. During rest, the bar fills with green. The yellow bar on the left indicates how much playtime is left in the ongoing round (max. $150 \mathrm{~s}$ ). B) Control game. The virtual hand is a green decagon that can be used to touch the pill-shaped, single-colored targets dropping in from the top of the screen, which then disappear with a delay of $1 \mathrm{~s}$ without producing a score. The green bar on the upper right fills up whenever the patient assumes the resting position.

plane. The second assessment measures the hand workspace of the trained limb (see the "Primary Outcome" section). While identical assessments are performed in both training groups, the system includes a specific version of a therapy game for each of the two training groups: (A) a rewarding version including monetary rewards, knowledge of performance feedback and graphical special effects (Figure 6.3A), and (B) a non-rewarding version lacking these motivators (Figure 6.3B). A more detailed description follows.

\section{Intervention}

In addition to standard therapy, both groups train 1 hour per day, 5 days a week for 3 weeks while inpatients in a participating rehabilitation hospital. Note that for study participants, standard therapy excludes additional proximal-arm training. All other therapies, however, are not affected by the study.

ArmeoSenso training is supervised by a therapist. Since 1 hour of consecutive upper limb training per day without weight support can be too demanding for some patients, deviations from this protocol are allowed to a minimum cumulative training time of $720 \mathrm{~min}$.

A typical ArmeoSenso training session is described in Wittmann et al. ${ }^{21}$ For the present study, patients log in to their user account with their random ID and the password printed on their butterfly etiquette. The IMUs are fixated to the affected lower and upper arm and to the trunk using custom-made Velcro straps (Balgrist Tec AG, Zurich, Switzerland). The supervising therapist may help if necessary. The ArmeoSenso system then guides the 
patient through three calibration poses and two automated assessments (see the "Outcome Measures" section) before training starts (beginning of the targeted 60 min session duration). In order to prevent physical exhaustion, the patient is visually instructed to rest for at least 4 seconds every 40 seconds. Moreover, patients are allowed to interrupt the training session if an additional break is needed. The duration of the additional breaks is added at the end of the training session. After $60 \mathrm{~min}$ of net training time, the automated assessments will be repeated and the patient will be asked to fill in a short motivation questionnaire (see the "Secondary Outcome" section).

Both groups train with modified versions of the ArmeoSenso "METEORS" game (see Wittmann et al. ${ }^{18}$ and Wittmann et al. ${ }^{21}$ ). Although the two versions differ markedly in terms of their appearance, they share the underlying game mechanics. That is, in both a virtual "hand" which matches the movement of the subject's real hand is used to catch objects that drop downwards from the top of the screen. The targets are placed within, or at the border of, the patient's virtual 3D workspace, which is continuously estimated and updated using a voxel-based model. ${ }^{18}$ The time to complete a round in the METEORS game is T_max $=150$ $\mathrm{s}$ (excluding rest). If, during these $150 \mathrm{~s}$, less than five targets were missed, the round is won and the difficulty increases by up to three levels, depending on the number of targets that hit the ground. Difficulty is adapted dynamically by changing (1) the average target speed of falling, (2) the target spawn interval and (3) the number of simultaneously spawned targets (one to a maximum of seven). It increases in this order $(1,2,3,1, \ldots)$. Conversely, difficulty decreases in reverse order if more than four targets were missed and the round is lost after a certain time ( $T_{-}$loss). In that case, the difficulty decrease is calculated by rounding $\frac{T_{-} m a x}{T_{-} \text {loss }}$ to the closest integer, but with a maximum of four difficulty levels.

\section{Rewarded training}

The reward group will train for 15 hours with a version of the METEORS game that is very similar to the one used in previous studies. ${ }^{18,21}$ Briefly, the hand is used to catch the targets that are depicted as meteors. The movement of the patient's whole arm is displayed with low latency on the computer screen as a moving virtual arm; a feature implemented to increase the feeling for embodiment and thus improve the motivation to move the arm. ${ }^{22}$ Subjects are instructed to use the hand to catch the falling meteors in order to protect their planet from being destroyed (Figure 6.3A). This game theme is easily understood and emotionally involving. ${ }^{21}$

Whenever a meteor is touched by the virtual hand, it explodes, giving the patient immediate knowledge of the result. Furthermore, a score appears with each exploding meteor that 
depends on the falling speed and diminishes with the time the meteor was visible on the screen before being caught. Scores are summed up over a round and reset when the next round starts. However, there is also an all-time high score always visible on the upper left (Figure 6.3A). If a meteor is missed, it crashes on the planet and damages it. Should the patient miss more than four meteors within T_loss $<150 \mathrm{~s}$, the round is lost, which results in visual effects showing the planet being destroyed and the camera shaking, followed by a message encouraging the patient to try again.

After successful level completion, patients are shown a feedback screen illustrating that they have successfully saved the planet, how many meteors they managed to catch and how many they have missed (Figure 6.4A). Monetary rewards are given for each completed level. Patients can win up to 1 Swiss Franc (CHF; approx. US\$ 1), if they succeed, but 0.1 $\mathrm{CHF}$ is deducted for every missed meteor. As a new level can be started approximately every 3 minutes, a maximum of $20 \mathrm{CHF}$ could be won per training session in case of an uninterrupted winning streak. This, however, is unlikely due to the difficult adaption described above. All of it, the money won during the preceding round, during the ongoing training session and the total money gathered over the whole course of the study, is presented on the feedback screen (Figure 6.4A), which is followed by a high score list showing the top 10 results (Figure 6.4B). If the current result was in the top 10, it is marked in the list (Figure 6.4B). This feature was also implemented to optimize patient engagement.

New planets (eight in total) and/or backgrounds (12 in total) are unlocked during the course of the three-week training. These rewards do not have any influence on the gameplay and difficulty but are intended to add variety to the game. Once three planets have been unlocked, the patient can choose between two randomly selected planets at the start of every round.
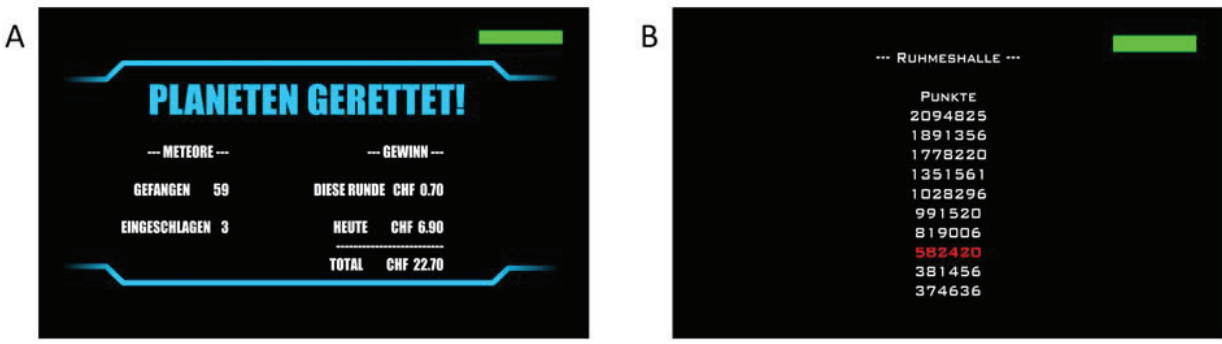

Figure 6.4: ArmeoSenso-Reward feedback screens. A) "PLANETEN GERETTET": planet saved. This screen is presented after each completed round. The number of meteors caught ("GEFANGEN", top) and meteors hitting the planet ("EINGESCHLAGEN", bottom) is indicated on the left. The monetary reward ("GEWINN") for the current round ("DIESE RUNDE", top), the current day ("HEUTE", middle) and the total amount of money gathered over the course of the study ("TOTAL", bottom) are displayed on the right. Note that a maximum of 1 Swiss Franc (CHF) can be won per round. B) Hall of fame ("RUHMESHALLE") with the patient's top 10 scores. If the current score is in the top 10 , it is highlighted in red. 


\section{Control training}

The control training consists of the same sensor system and game mechanics with all rewarding feedback removed. In order to reduce the feeling of embodiment, ${ }^{22}$ only the position of the hand is shown as a green decagon on a plain black background. Targets are simple pill-shaped, single-colored objects that disappear with a delay of $1 \mathrm{~s}$ without producing a score or sound after being touched; hence, there is no immediate but delayed knowledge of performance. Complete removal of knowledge of performance is not possible in this game because patients then might reach for the same target for several times, which would hamper comparability to the other study group. The feedback screen, the monetary reward, the high score list and the unlocking of new planets and backgrounds are also removed. Instead, patients are looking at a blank screen to keep the training time comparable. Most notably, the target placement and difficulty adaption remain unaffected.

\section{Outcome measures}

The clinical assessments are collected by assessors blinded to treatment allocation. All assessors are trained in performing the assessments before the start of the trial. In addition to the outcome measures described below, demographics, comorbidities, cognitive function (Mini Mental State Examination) and concomitant therapy will be recorded (Figure 6.5).

\section{Primary outcome}

The primary outcome of this trial is the workspace of the impaired arm in the horizontal plane, measured by using an assessment integrated into the ArmeoSenso platform. Subjects are instructed to actively reach out as far as possible with their impaired arm forward, backward and sideways to explore the entire arm workspace. The workspace is corrected for trunk movements and computed as the number of square pixels of $10 \mathrm{~cm}$ side length arranged in the transverse plane relative to the patient's trunk (Figure 6.2B) (see Wittmann et al. ${ }^{18}$ and Wittmann et al. ${ }^{21}$ for more information). This assessment is conducted immediately before and after every therapy session (Figure 6.5).

\section{Secondary outcome}

Arm impairment is assessed using the Fugl-Meyer Assessment - Upper Extremity (FMAUE), arm activity using the Wolf Motor Function Test (WMFT), the Box and Block Test, and a pointing task (ArmeoSenso integrated assessment) (Figure 6.5). For the pointing task, nine targets arranged in two semicircles appear one after another in the transversal 


\begin{tabular}{|c|c|c|c|c|c|c|}
\hline & \multicolumn{6}{|c|}{ STUDY PERIOD } \\
\hline & Enrolment & $\begin{array}{c}\text { Baseline } \\
\text { assessment }\end{array}$ & Allocation & Training & $\begin{array}{c}\text { Post-training } \\
\text { assessment }\end{array}$ & $\begin{array}{l}3 \text { Month } \\
\text { Follow-up }\end{array}$ \\
\hline TIMEPOINT & Day 0 & Day 1 & Day 2 & Day 2-22 & Day $23 \pm 5$ & Day $113 \pm 7$ \\
\hline ENROLMENT: & & & & & & \\
\hline Eligibility screen & $\mathrm{x}$ & & & & & \\
\hline Informed consent & $\mathrm{x}$ & & & & & \\
\hline $\begin{array}{l}\text { Randomization and } \\
\text { allocation }\end{array}$ & & & $\mathrm{x}$ & & & \\
\hline INTERVENTIONS: & & & & & & \\
\hline Rewarded training & & & & $\mathrm{x}$ & & \\
\hline Control training & & & & $\mathrm{x}$ & & \\
\hline ASSESSMENTS: & & & & & & \\
\hline Demographics & & $\mathrm{x}$ & & & & \\
\hline Comorbidities & & $\mathrm{x}$ & & & & \\
\hline $\begin{array}{l}\text { Mini-Mental State } \\
\text { Examination }\end{array}$ & & $\mathrm{X}$ & & & & \\
\hline Concomitant therapy & & $x$ & & & & $\mathrm{x}$ \\
\hline Primary outcome: & & & & & & \\
\hline $\begin{array}{l}\text { Workspace } \\
\text { assessment }\end{array}$ & & & & $\mathrm{x}$ & & \\
\hline Secondary outcomes: & & & & & & \\
\hline $\begin{array}{c}\text { Fugl-Meyer } \\
\text { Assessment - Upper } \\
\text { Extremity }\end{array}$ & & $\mathrm{x}$ & & & $\mathrm{x}$ & $\mathrm{x}$ \\
\hline $\begin{array}{c}\text { Wolf Motor Function } \\
\text { Test }\end{array}$ & & $\mathrm{x}$ & & & $\mathrm{x}$ & $\mathrm{x}$ \\
\hline Box and Block Test & & $x$ & & & $\mathrm{x}$ & $\mathrm{x}$ \\
\hline $\begin{array}{l}\text { Motor Activity } \\
\text { Log }-14\end{array}$ & & $\mathrm{x}$ & & & $\mathrm{x}$ & $\mathrm{x}$ \\
\hline Barthel Index & & $\mathrm{x}$ & & & $\mathrm{x}$ & $\mathrm{x}$ \\
\hline $\begin{array}{l}\text { National Institutes of } \\
\text { Health Stroke Scale }\end{array}$ & & $\mathrm{X}$ & & & $\mathrm{X}$ & $\mathrm{x}$ \\
\hline modified Rankin Scale & & $\mathrm{x}$ & & & $\mathrm{x}$ & $\mathrm{x}$ \\
\hline Pointing task & & & & $\mathrm{x}$ & & \\
\hline $\begin{array}{l}\text { Motivation } \\
\text { questionnaire }\end{array}$ & & & & $\mathrm{x}$ & & \\
\hline Adverse events: & & $x$ & & $\mathrm{x}$ & $\mathrm{x}$ & $\mathrm{x}$ \\
\hline
\end{tabular}

Figure 6.5: Recommendation for Interventional Trials (SPIRIT) figure: the schedule of enrolment, interventions, and assessments. MT, month; MMSE, Mini-Mental State Examination; FMA-UE, Fugl-Meyer Assessment of the upper extremity; WMFT, Wolf Motor Function Test; MAL-14, Motor Activity Log 14; BI, Barthel Index; NIHSS, National Institutes of Health Stroke Scale; mRS, modified Rankin Scale.

plane in front of the subject. The goal is to reach out to the target within $8 \mathrm{~s}$. The number of targets reached and the mean time to target is reported. The Motor Activity Log 14 (MAL-14) for self-reported movement ability, the Barthel Index (BI) as a measure of 
independence in daily living, the National Institutes of Health Stroke Scale (NIHSS) as a measure of stroke severity are recorded and the global disability is assessed using the modified Rankin Scale (mRS) (Figure 6.5).

Finally, patients fill in a short questionnaire after each training session. Ten questions (five positively and five negatively formulated), given in randomized order, evaluate the subjective appraisal of the training on a five-point Likert-scale (Figure 6.5).

\section{Assessments of safety}

Adverse events (AEs) expected to occur are skeletal or muscular pain and fatigue indicating a syndrome of overuse. The quality management system of the Clinical Trail Center Zurich will be followed according to national and international guidelines. ${ }^{23}$ Adverse events (AEs) will be documented and related serious adverse events (SAEs) will be reported to the Ethical Committee, the competent authority (Swissmedic) and local principle investigators (PIs). All SAEs will be included in an annual report to authorities and PIs. AEs will be recorded from baseline assessment to the end of the trial.

\section{Sample size}

The sample size is estimated to detect a between-group difference of 4.8 voxels in the workspace difference from beginning to end of training, based on the improvement in arm workspace from pilot results (unpublished) and an estimated group difference of $20 \%$. This assumes a two-sided alpha level at 0.05 and a power of $80 \%$. For an effect with a standard deviation of seven voxels, 35 subjects per group yields $80 \%$ power to detect the true alternative. We will randomize 37 subjects in each group, based on our observed attrition rate of $5 \%$ in a previous interventional trial. ${ }^{21}$ This calculation was performed using $\mathrm{G}^{\star}$ Power 3.1.24,25

\section{Statistical analysis}

Our primary analysis is an intention-to-treat analysis comparing the two groups. In addition, as this is an explanatory trial, a per-protocol analysis will be used to analyse the effect of feedback under ideal conditions. ${ }^{26,27}$ Therapy will take place in 15 sessions over 3 weeks, and there is the possibility that some subjects will not complete the full treatment regimen due to scheduling issues or other time constraints. If they still perform at least 12 hours of therapy the data will be analysed. All other patients will be considered "noncompliant" in the sense that they do not receive the full treatment dose. According to the per-protocol principle, their outcomes will not be analysed. 
A two-sample t-test comparing the mean change in voxel workspace assessment between the two groups will be used; in case of non-normality, a Mann-Whitney test will be computed instead. Moreover, repeated measures analysis of variance (ANOVAs), in case of normally distributed data, or non-parametric Friedman tests will be used to assess the development of the different outcome measures over time. Statistical significance will be based on a p-value threshold of 0.05. Data will be analysed using MATLAB R2013b (or newer) (Mathworks Inc., Natick, MA, USA) and SPSS (version 23 (or newer), IBM Corp., Armonk, NY, USA).

\section{DISCUSSION}

This is the first randomized clinical trial to evaluate the effect of enhanced feedback and reward on arm rehabilitative training following stroke. Intrinsic (score, knowledge of performance) and extrinsic rewards (money) hypothetically improve motor cortex plasticity and overall motivation to train. Because motivation affects training time and time is a crucial determinant of effect, ${ }^{4}$ this trial controls for time by using a control intervention that is matched in time and dose of training.

In a motor learning study with healthy young subjects, we have shown that the consolidation/retention of a skilled motor task is more effective if the task was trained in the presence of reward. ${ }^{10}$ In a rat model, projections from midbrain dopaminergic regions to $\mathrm{M} 1$ are required for successful motor learning and functional plasticity at cortical (layer II/III) synapses, ${ }^{15,16}$ mechanisms that presumably support recovery after stroke. ${ }^{28}$ Whether the dopaminergic system can be stimulated to improve recovery remains to be shown. Likewise, whether reward is an appropriate stimulus is yet unknown.

Previous studies have assessed the patient's motivation for a specific training (e.g. Wittmann et al. ${ }^{21}$ and Nijenhuis et al. ${ }^{29}$ ), but none of them compared the outcome to an appropriate control condition for the evaluation of the effectiveness of rewarding therapy. Although functional improvement itself might be motivating enough for some patients to train, here we are in search of a clinical effect of reward on a reduction in impairment (shoulder/elbow range of motion (ROM)) mediated by active and repetitive proximal-arm training. We chose this training method because (1) it can be standardized in its conduct and has quantifiable parameters of dose, movement success and arm workspace as primary outcome measure, (2) it is based on a therapy system which was already evaluated with patients and found to be safe, (3) it is easily supported in participating institutions without much training of therapists who provide assistance to the patient and (4) it has shown a moderate effect on chronically arm-impaired stroke survivors. ${ }^{21}$ Because the ArmeoSenso training only works 
on proximal-arm function, it is not expected to have a clinically relevant effect on activities of daily living, independence, or quality of life. We therefore chose a primary outcome that is close to what is actually being trained, i.e., arm workspace. Workspace assessments have been widely used to assess the arm function of stroke patients, thereby showing high correlation to standard clinical scales. ${ }^{30,31}$ For a discussion of clinical relevance of functional outcomes, in the interest of clarity and conciseness, we would like to refer to the review of Ashford, Slade, Malaprade, Turner-Stokes. ${ }^{32}$ Nevertheless, potential transfer to more clinical scores can be tracked using our secondary outcome measures.

The study is enrolling subjects during the initial three months after stroke. Most recovery is occurring in this period. ${ }^{33-37}$ Therefore, we expect an improvement in arm function in both groups.

A positive outcome of this trial will emphasize the role of reward in rehabilitative training. This result could potentially be applicable to various forms of post-stroke rehabilitative training. Social rewards (smileys, praise), food rewards (sweets, dietary allowance), monetary reward or token programs are options that are easy to implement in situations where there is systematic interaction between a patient and a human trainer or a technical training device. Virtual-reality-based training games, therapy elements including repetitive performance feedback and similar approaches are examples where to integrate reward according to suggestions to be derived from the described study.

\section{Trial status}

The trial is currently recruiting patients. At the time of submission, 16 stroke patients have been enrolled.

\section{Declarations}

\section{Ethics approval and consent to participate}

The study will follow GCP-guidelines and has been approved by the responsible local ethics committees "Ethikkommission Nordwest- und Zentralschweiz" and the "Kantonale Ethikkommission Zürich" (LU2013-079 and PB_2016-01804), and the Swiss Agency for Therapeutic Products (Swissmedic: 2014-MD-0033). All subjects have to give written informed consent in accordance with the Declaration of Helsinki. 


\section{Consent for publication}

Written informed consent was obtained from the participant (Figure 6.2A) for publication of this photograph in this manuscript. The consent form is held by the authors and is available for review by the Editor-in-Chief.

\section{Acknowledgements}

The authors would like to thank Mark van Raai for his help in the implementation of the ArmeoSenso software, and Irene Christen, Jose Lopez and Belen Valladares for their support with the study.

\section{REFERENCES}

1. Parker VM, Wade DT, Langton Hewer R. Loss of arm function after stroke: measurement, frequency, and recovery. International Rehabilitation Medicine. 1986;8(2):69-73.

2. Kwakkel G, Kollen BJ, van der Grond J, Prevo AJ. Probability of regaining dexterity in the flaccid upper limb: impact of severity of paresis and time since onset in acute stroke. Stroke. 2003;34(9):2181-2186.

3. Nichols-Larsen DS, Clark PC, Zeringue A, Greenspan A, Blanton S. Factors influencing stroke survivors' quality of life during subacute recovery. Stroke. 2005;36(7):1480-1484.

4. Veerbeek JM, van Wegen E, van Peppen R, van der Wees PJ, Hendriks E, Rietberg M, Kwakkel G. What is the evidence for physical therapy poststroke? A systematic review and meta-analysis. Plos One. 2014;9(2).

5. Cooke EV, Mares K, Clark A, Tallis RC, Pomeroy VM. The effects of increased dose of exercisebased therapies to enhance motor recovery after stroke: a systematic review and meta-analysis. BMC Medicine. 2010;8:60.

6. Kwakkel G. Impact of intensity of practice after stroke: issues for consideration. Disability and Rehabilitation. 2006;28(13-14):823-830.

7. Kwakkel G, Kollen BJ, Krebs HI. Effects of robot-assisted therapy on upper limb recovery after stroke: a systematic review. Neurorehabilitation and Neural Repair. 2008;22(2):111-121.

8. Wachter T, Lungu OV, Liu T, Willingham DT, Ashe J. Differential effect of reward and punishment on procedural learning. Journal of Neuroscience. 2009;29(2):436-443.

9. Abe M, Schambra H, Wassermann EM, Luckenbaugh D, Schweighofer N, Cohen LG. Reward improves long-term retention of a motor memory through induction of offline memory gains. Current Biology. 2011;21(7):557-562.

10. Widmer M, Ziegler N, Held J, Luft A, Lutz K. Rewarding feedback promotes motor skill consolidation via striatal activity. Progress in Brain Research. 2016.

11. Galea JM, Mallia E, Rothwell J, Diedrichsen J. The dissociable effects of punishment and reward on motor learning. Nature Neuroscience. 2015;18(4):597-602.

12. Knutson B, Delgado MR, Phillips PE. Representation of subjective value in the striatum. Neuroeconomics: Decision making and the brain. 2008:398-406.

13. Lutz K, Pedroni A, Nadig K, Luechinger R, Jancke L. The rewarding value of good motor performance in the context of monetary incentives. Neuropsychologia. 2012;50(8):1739-1747.

14. Schott BH, Minuzzi L, Krebs RM, Elmenhorst D, Lang M, Winz OH, Seidenbecher CI, et al. Mesolimbic functional magnetic resonance imaging activations during reward anticipation correlate with reward-related ventral striatal dopamine release. Journal of Neuroscience. 2008;28(52):14311-14319. 
15. Hosp JA, Pekanovic A, Rioult-Pedotti MS, Luft AR. Dopaminergic projections from midbrain to primary motor cortex mediate motor skill learning. Journal of Neuroscience. 2011;31(7):24812487.

16. Molina-Luna K, Pekanovic A, Rohrich S, Hertler B, Schubring-Giese M, Rioult-Pedotti MS, Luft AR. Dopamine in motor cortex is necessary for skill learning and synaptic plasticity. PLoS One. 2009;4(9):e7082.

17. Krakauer JW. Motor learning: its relevance to stroke recovery and neurorehabilitation. Current Opinion in Neurology. 2006;19(1):84-90.

18. Wittmann F, Lambercy O, Gonzenbach RR, van Raai MA, Hover R, Held J, Starkey ML, et al. Assessment-driven arm therapy at home using an IMU-based virtual reality system. Paper presented at: International Conference on Rehabilitation Robotics (ICORR) 2015.

19. Boutron I, Moher D, Altman DG, Schulz KF, Ravaud P, Group C. Extending the CONSORT statement to randomized trials of nonpharmacologic treatment: explanation and elaboration. Annals of Internal Medicine. 2008;148(4):295-309.

20. Chan AW, Tetzlaff JM, Altman DG, Laupacis A, Gotzsche PC, Krleza-Jeric K, Hrobjartsson A, et al. SPIRIT 2013 statement: defining standard protocol items for clinical trials. Annals of Internal Medicine. 2013;158(3):200-207.

21. Wittmann F, Held JP, Lambercy O, Starkey ML, Curt A, Hover R, Gassert R, et al. Self-directed arm therapy at home after stroke with a sensor-based virtual reality training system. Journal of NeuroEngineering and Rehabilitation. 2016;13(1):75.

22. Price TF, Peterson CK, Harmon-Jones E. The emotive neuroscience of embodiment. Motivation and Emotion. 2012;36(1):27-37.

23. ICH. ICH Harmonised Tripartite Guideline. Guideline for Good Clinical Practice E6(R1). 1996.

24. Faul F, Erdfelder E, Buchner A, Lang AG. Statistical power analyses using G*Power 3.1: tests for correlation and regression analyses. Behavior Research Methods. 2009;41(4):1149-1160.

25. Faul F, Erdfelder E, Lang AG, Buchner A. G*Power 3: a flexible statistical power analysis program for the social, behavioral, and biomedical sciences. Behavior Research Methods. 2007;39(2):175-191.

26. Sedgwick P. Explanatory trials versus pragmatic trials. British Medical Journal. 2014;349:g6694.

27. Sedgwick P. Intention to treat analysis versus per protocol analysis of trial data. British Medical Journal. 2015;350:h681.

28. Nudo RJ. Adaptive plasticity in motor cortex: implications for rehabilitation after brain injury. Journal of Rehabilitation Medicine. 2003(41 Suppl):7-10.

29. Nijenhuis SM, Prange GB, Amirabdollahian F, Sale P, Infarinato F, Nasr N, Mountain G, et al. Feasibility study into self-administered training at home using an arm and hand device with motivational gaming environment in chronic stroke. Journal of NeuroEngineering and Rehabilitation. 2015;12(1):1.

30. Bailey RR, Klaesner JW, Lang CE. Quantifying real-world upper-limb activity in nondisabled adults and adults with chronic stroke. Neurorehabil Neural Repair. 2015;29(10):969-978.

31. van Meulen FB, Reenalda J, Buurke JH, Veltink PH. Assessment of daily-life reaching performance after stroke. Annals of Biomedical Engineering. 2015;43(2):478-486.

32. Ashford S, Slade M, Malaprade F, Turner-Stokes L. Evaluation of functional outcome measures for the hemiparetic upper limb: a systematic review. Journal of Rehabilitation Medicine. 2008; 40(10):787-795.

33. Prabhakaran S, Zarahn E, Riley C, Speizer A, Chong JY, Lazar RM, Marshall RS, et al. Interindividual variability in the capacity for motor recovery after ischemic stroke. Neurorehabilitation and Neural Repair. 2008;22(1):64-71. 
34. Zarahn E, Alon L, Ryan SL, Lazar RM, Vry MS, Weiller C, Marshall RS, et al. Prediction of Motor Recovery Using Initial Impairment and fMRI 48 h Poststroke. Cerebral Cortex. 2011; 21(12):2712-2721.

35. Zeiler SR, Gibson EM, Hoesch RE, Li MY, Worley PF, O’Brien RJ, Krakauer JW. Medial premotor cortex shows a reduction in inhibitory markers and mediates recovery in a mouse model of focal stroke. Stroke. 2013;44(2):483-489.

36. Krakauer JW, Carmichael ST, Corbett D, Wittenberg GF. Getting neurorehabilitation right: What can be learned from animal models? Neurorehabilitation and Neural Repair. 2012; 26(8):923-931.

37. Murphy TH, Corbett D. Plasticity during stroke recovery: from synapse to behaviour. Nature Reviews Neuroscience. 2009;10(12):861-872. 



\section{Chapter 7}

\section{Autonomous rehabilitation at stroke patients home for balance and gait: safety, usability and compliance of a virtual reality system}

J.P. Held, B. Ferrer, R. Mainetti, A. Steblin, B. Hertler, A. Moreno-Conde, A. Duenas, M. Pajaro, C.L. Parra-Calderon, E. Vargiu, M.J. Zarco, M. Barrera, C. Echevarria, F. Jodar-Sanchez, A.R. Luft, N.A. Borghese 


\section{ABSTRACT}

Background New technologies, such as telerehabilitation and gaming devices offer the possibility for patients to train at home. This opens the challenge of safety for the patient as he is called to exercise neither with a therapist on the patients' side nor with a therapist linked remotely to supervise the sessions.

Aim To study the safety, usability and patient acceptance of an autonomous telerehabilitation system for balance and gait (the REWIRE platform) in the patient's home.

Design Cohort study.

Setting Community, in the stroke patients' home.

Population 15 participants with first-ever stroke, with a mild to moderate residual deficit of the lower extremities.

Methods Autonomous rehabilitation based on virtual rehabilitation was provided at the participants' home for twelve weeks. The primary outcome was compliance (the ratio between days of actual and scheduled training), analysed with the twotailed Wilcoxon Mann-Whitney test. Furthermore safety is defined by adverse events. The secondary endpoint was the acceptance of the system measured with the Technology Acceptance Model. Additionally, the cumulative duration of weekly training was analysed.

Results During the study there were no adverse events related to the therapy. Patients performed on average $71 \%$ (range 39 to $92 \%$ ) of the scheduled sessions. The Technology Acceptance Model Questionnaire showed excellent values for stroke patients after the training. The average training duration per week was $99 \pm 53 \mathrm{~min}$.

Conclusions Autonomous telerehabilitation for balance and gait training with the REWIRE-system is safe, feasible and can help to intensive rehabilitative therapy at home.

Clinical rehabilitation impact Telerehabilitation enables safe training in home environment and supports of the standard rehabilitation therapy.

Trial registration URL: https://clinicaltrials.gov. Unique identifier: NCT02192125. Registered 30 May 2014 (retrospectively registered). 


\section{BACKGROUND}

A key factor for successful neurorehabilitation after stroke is intensive training. ${ }^{1}$ Intensity is defined by the time spent in training and the training demand, i.e., its complexity, level of difficulty and challenge for the trainee. This is optimized for being inpatient. ${ }^{2}$ After being discharged home, most stroke patients still suffer from a residual impairment that requires additional therapy to improve further or to prevent deterioration. ${ }^{3}$ This additional therapy could be offered in outpatient centers or at patients' home (domiciliary therapy), which requires a large organization effort and strain. ${ }^{4}$ Besides this, economical support for outpatient rehabilitation by service providers has become very limited due to budget cuts. ${ }^{3,5,6}$ Moreover, in most cases, outpatient therapy does not reach intensity sufficient to produce meaningful effects. ${ }^{1,7}$

Information and communication technologies (ICT) have the potential to improve this situation $^{9-13}$ by enabling the video/audio communication between a healthcare professionals and the patient at home. Results generally show a fragmentation in protocols and not enough evidence for a benefit for the patients. ${ }^{13}$ Moreover, economical gains are limited, as a therapist has still to be present remotely.

A different approach to telerehabilitation has been offered by commercial gaming devices like the Nintendo balance board and the Microsoft Kinect used to track human motion in real-time with an acceptable accuracy. ${ }^{14,15}$ In combination with powerful game engines like Panda3D and Unity3D, they have been recently explored to provide autonomous rehabilitation at the patient's home in the form of games. ${ }^{8,16,17}$

These studies have shown that such an approach can be effective in improving patient's motor ability and motivating, and even more effective than standard rehabilitation. ${ }^{18}$ However, such approach opens the challenge of safety for the patient as he is called to exercise neither with a therapist on the patients' side nor with a therapist linked remotely to supervise the sessions. A recent pilot clinical studies have reported adverse events (e.g., Prosperini et al. ${ }^{19}$ - occurrence of knee or lower back pain that limited compliance) that have caused patients dropping out of the studies.

Recently, inside the European Commission funded project REWIRE, a platform that integrates inside the game engine a continuous monitoring of patient's motion is provided: ${ }^{20}$ motion data are analysed in real-time, to diminish the risk for occurrence of therapy-related adverse events. This allows preventing maladaptation and/or joints overloading, thereby making therapy unsupervised by the therapist, safe for the patient. This real-time analysis is used to provide immediate on-line feedback by the system about movement quality and suggestions to correct it. At the end of each training session, a summary feedback on 
how well the exercise was carried out (online tutor) as well as on how much progress was achieved is provided to the patient.

Such an approach would allow one step forward in telerehabilitation as it would allow safe exercising at home. Here, we present the usability results of a home autonomous therapy system (the REWIRE platform ${ }^{[1]}$ ).

\section{METHODS}

\section{Description of the platform}

The REWIRE autonomous telerehabilitation platform is aimed at allowing patients to continue intensive rehabilitation at home under asynchronous remote monitoring by clinicians working at the hospital. The platform consists of three hierarchical components: Patient Station, Hospital Station, and Networking Station.

Patient Station, Computer installed at patient's home and a TV screen, to play rehabilitative balance exergames (a video game that is also a form of exercise) and collects game information and motion data. The Patient Station in this study has been configured for posture rehabilitation and it includes a force plate (Tymo, Tyromotion, Graz, Austria), a 3D camera (Kinect, Microsoft Inc, Redmond USA) and a host computer connected to a TV screen, ${ }^{20}$ to the Kinect camera via USB port and to the force plate through Bluetooth wireless connection.

Control of the Patient Station software is implemented by gesture tracking, therefore no keyboard or mouse input is necessary. ${ }^{21,22}$ Exergames are pre-selected by a clinician at the Hospital Station and, each day at training start, they are proposed to the patient by the virtual therapist, embedded insight the game engine, are presented to the patient. The patient is free to choose which game to start with.

The patient exercises in front of the TV screen and the movement is tracked by the Kinect camera $^{23}$ and used to animate the avatar. For some exergames, a force plate is used to track the Centre of Pressure; ${ }^{24}$ this is projected down to the virtual floor of the game to provide feedback to the patient. In the exergame, the patient sees himself as an avatar moving and interacting in real-time with the virtual game environment (Figure 7.2D, and Figure 7.3). The virtual therapist guides the patients through each therapy session, including advice and encouragement during the exergames; at the end of each daily session it summarizes the results.

[1] http://www.rewire-project-eu 
The Virtual Therapist is endowed with an artificial intelligence engine based on a fuzzy control system. ${ }^{22}$ This analyses motion and pressure data during the training sessions and is able to inform the patient about wrong movements that are potentially harmful and may lead to maladaptation or strain injuries. ${ }^{19,25}$ The fuzzy system is instructed by clinicians before starting the therapy. The correctness of the posture maintained while performing the exercise is clearly shown in real-time through a meaningful color code painted over the 3D avatar (Figure 7.1). Each body segment color could change from an intense green (correct posture) to a red colour (wrong posture) passing through the spectrum of colors (yellow and orange). If a wrong posture is detected, the exergame is paused and the Virtual Therapist avatar pops-up and explains the correct way to perform the movements required by the game (Figure 7.2). ${ }^{22}$ To this aim, a simple face animation of the virtual therapist is used to improve engagement and compliance. ${ }^{26}$ In the extreme cases, when the movement is dangerous, the system forces the shutdown and sends a message to the therapist (this never occurred in the pilot). Music, selected by the patient, is played during the game to avoid isolation and increase compliance.
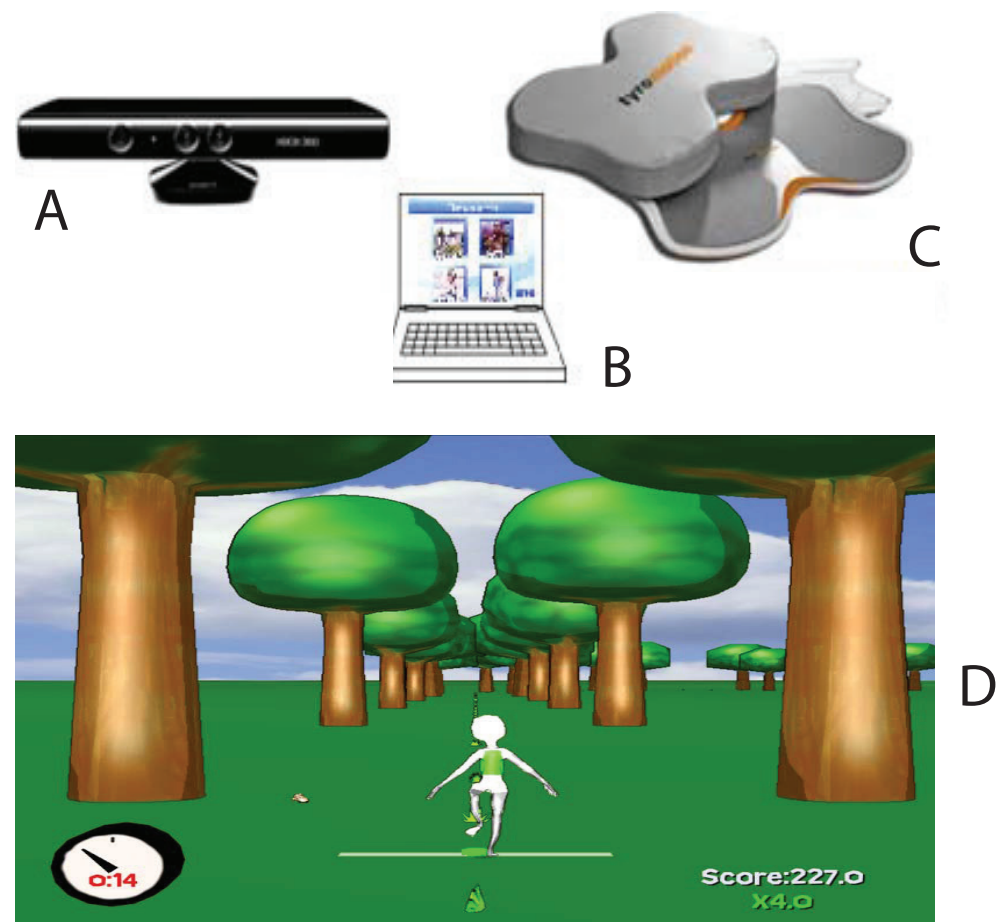

Figure 7.1: Patient station components and deployment for one patient.

A) Microsoft Kinect Camera; B) Laptop; C) Tyromotion Balance Board; D) Screenshot of one of the exergames: "animal hurdler". Notice the green spot under the avatar: this shows the position of the center of pressure (CoP) that indicates the resulting of all forces exerted on the ground. In a healthy postural control, the CoP lies in between the feet when standing vertically. 


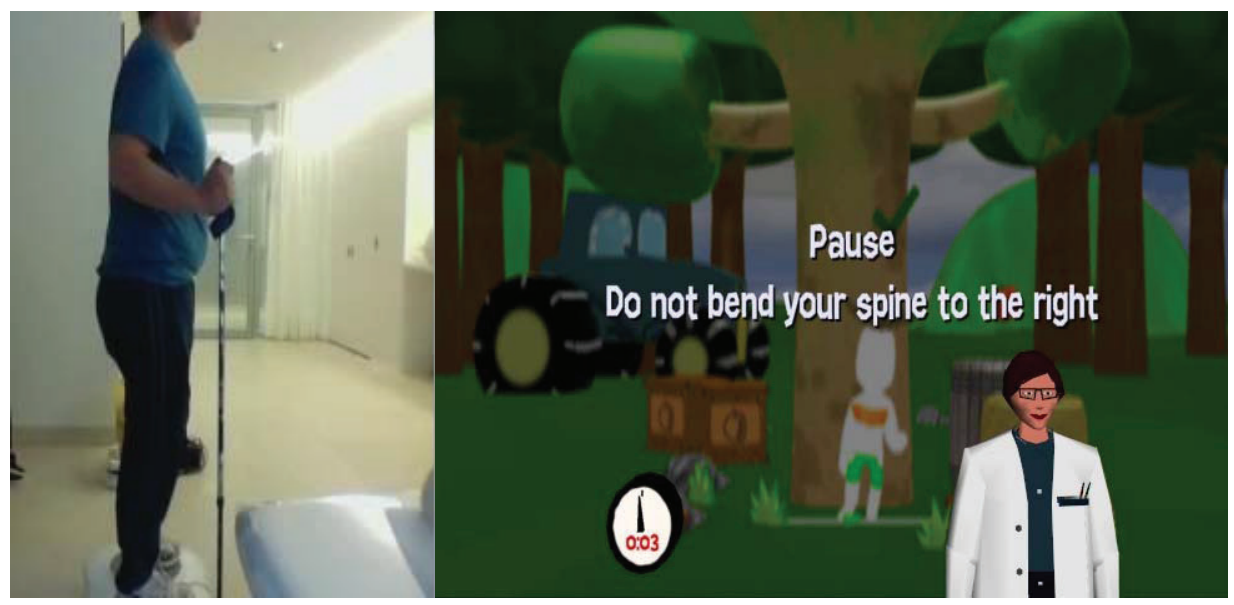

Figure 7.2: Playing patient.

A patient playing fruit catcher game is bending his spine on the right too much and he is advised by the Virtual Therapist.

A set of eleven exergames have been designed starting from therapists' specifications according to pre-specified guidelines ${ }^{27}$ to implement exercises to train body alignment, weight shifting, stepping, raising legs and sit to stand (see Pirovano, Mainetti for detailed description of the exergame). ${ }^{22}$ The Patient Station logs the gaming data: the motion of the patient's skeleton, the time course of the Center Of Pressure and the interaction with game elements are recorded and sent to the Hospital Station at the end of each session for assessment by a clinician.

The Hospital Station installed in the rehabilitation center to configure, schedule and review therapy sessions by a clinician. The Hospital Station is a cloud-based service that allows therapists, through a Web graphical interface, to schedule and personalizes the rehabilitation sessions and revise rehabilitation results, any time, from anywhere, also outside the hospital. It has been designed with therapists to maximize compliance and it is organized into two main modules: therapy schedule and assessment. The modules are structured through a set of hierarchical interfaces to maximize ease of use. The scheduler is based on a calendar: it allows defining the mix of exercises that the patient is required to carry out daily. Such exercises can be grouped into sessions to make assignment easier.

A critical aspect specific of exergames for rehabilitation is the regulation of the degree of difficulty: this should be adapted to the actual patient capabilities. When an exercise is too easy, it has little effect, if exercise is too difficult, it may become frustrating. The Hospital Station allows regulating for each exergame its speed, range of motion and/or accuracy. 
To simplify the exercises, a set of predefined difficulty levels is offered to the therapist. The regulation of the difficulty enables implementing a progressive therapy schedule as a continuous learning stimulus. ${ }^{28,29}$ The exergame parameters are for instance the amplitude of apples falling from a tree (amplitude control), the speed of a tractor moving in the virtual field (velocity control).

The assessment module shows the patient's performance, through a specific Web interface integrated inside the Hospital Station. It shows a summary table of the compliance with the therapy along with a detailed description of the patient's performance. The movements executed during each session can also be played back through a skeleton to better understand how the exercise was performed along with the time course of the Center of Pressure on the floor plane. The same Web interface allows regulating monitoring. The maximum value of specific parameters is defined (e.g. spine bending, knee flexion, displacement of the Center of Pressure), thus defining for those parameters a range of admissible values. The range is mapped on a continuous scale from 0 to 4 . The interval of values [0-2) is associated to correct movement (green avatar), the interval [2-3) to warning level (yellow avatar), the interval [3-4) to dangerous movement (red avatar, the exergame is paused). If the value is equal or above 4, the exercise is interrupted for safety reasons, the system shuts down and a message is sent to the therapist.

The Networking Station, at the health provider site, mines the data to discover common features and trends of rehabilitation treatment among hospitals and regions. Moreover, analysing over time the recovery curve of each patient and the mix of exercises provided, it could determine which exercises could be most effective for particular groups of patients.

\section{Therapy scheduling}

At the beginning of the therapy program, the therapist defined a weekly set of exercises to be performed daily and tailored them to the patient status choosing the adequate level of difficulty and activates specific monitoring. Each session was composed of a mix of exercises that often involve equilibrium, coordination and increase of lower limbs lower limb muscle strength. ${ }^{3}$ The sessions were scheduled to last between 10 to 40 minutes per day depending on the patients' capacity and the therapist knowledge of performing each exercise. Based on the data collected by the Patient Station during the prior week and on the qualitative report from the weekly visit by the therapist to the patient's home, the clinician could update inside the Hospital Station the program for the following week by adjusting game mix selection, difficulty level, monitoring, and session duration. 


\section{Installing the REWIRE telerehabilitation platform at home and follow-up}

Swiss patients were trained on the Patient Station for one day at the hospital. Spanish participants received this training during the last two weeks of their outpatient program. Afterwards, the Patient Station was installed at the patient's home, and the autonomous home training was continued for three consecutive months.

When a problem occurred, the patient contacted the therapist by telephone. If the problem could not be solved in the first line, a therapist or technician visited the patient. In case the problem persisted, a remote connection through TeamViewer software (Permira, London, UK) was established with the technical team in the University of Milan to solve problems.

\section{Design of the study}

The pilot study was designed to evaluate safety, feasibility and compliance of the REWIRE intervention in stroke patients. This study was performed in the stroke patients' homes and assessments where performed at the cereneo, Centre for Neurology and Rehabilitation (Vitznau, Switzerland) and at the Virgen del Rocio University Hospital (Seville, Spain). Patients recruited had already completed their outpatient rehabilitation program and did not receive any rehabilitation training any more. The study followed "Good Clinical Practice (GCP)" guidelines ${ }^{30}$ and was approved by the responsible ethics committees for each site.

\section{Participants}

First-time stroke patients with a mild to moderate residual deficit of the lower extremities were recruited (Functional Ambulatory Categories $\geq 3 ;^{31,32}$ Berg-Balance-Scale (BBS) $\geq$ $\left.21^{33}\right)$. All patients were willing to train at home with an autonomous telerehabilitation system for balance and gait (the REWIRE platform). Exclusion criteria were presence of aphasia, dementia, severe neglect or other neurological disease.

\section{Outcome measures}

Outcome was assessed at baseline, before the intervention, and after the intervention, by a trained therapist. The primary safety outcome was related adverse events (e.g. pain, falls) or serious adverse event during the study period. The acceptance of the intervention was evaluated through the compliance defined by the ratio between days of actual and scheduled training. The reason for skipping a scheduled session was recorded by the supporting therapist. Additionally the cumulative duration of weekly training was analysed. 
Safety measure, defined in the system during the sessions was evaluated through the mean value of monitoring occurring during the training sessions, ranging from 0 (correct movement) to 4 (dangerous movement).

Patients' acceptance of the telerehabilitation program was measured using the Technology Acceptance Model (TAM) questionnaire. ${ }^{34-37}$ TAM is one of the most commonly used measures of user acceptance and usage of technological devices along the following dimensions: perceived ease of use, perceived usefulness and attitude toward using and behavioural intention to use the system..$^{38}$ The TAM questionnaire has been tailored to the REWIRE intervention by taking also into account the patient's involvement in the program, knowledge of disability and satisfaction. It comprises 24 items (see Appendix A) that are rated in a seven-point Likert-scale, whereby a score of one refers to "I do not agree at all" and a score of seven refers to "I agree entirely". Patients receiving autonomous telerehabilitation training evaluated their acceptance after the initial two weeks of training and again after three months.

\section{Statistics}

For statistical analysis we used Prism version 6 (GraphPad Prism, La Jolla, USA). Descriptive statistics are reported as median \pm interquartile range [IQR]. Two-sided $\mathrm{P}$ values less than 0.05 were considered significant. The two-tailed Wilcoxon Mann-Whitney test was used to compare the average weekly training duration in patients with higher risk of falling against patients with lower risk of falling, based on the BBS.

\section{RESULTS}

\section{Recruited participants}

16 subjects with first-ever stroke ( 3 to 74 months post-stroke) and mild to moderate impairments in balance and gait (BBS: 47 [43; 53]) were recruited between April 2014 and February 2015. All subjects gave informed consent (6 in Switzerland and 9 in Spain). The study followed GCP-guidelines and was approved by the local Cantonal ethics committee "Nordwest- und Zentralschweiz" (EKNZ: 2014-061) and by the ethical committee from Virgen del Rocío Hospital in Seville, Spain (CEIcode 2012PI/216). Baseline demographics and baseline functional parameters are shown in Table 7.1. 
Table 7.1: Baseline characteristics

\begin{tabular}{lccc}
\hline Characteristic & $\begin{array}{c}\text { Switzerland } \\
\text { Median, IQR }\end{array}$ & $\begin{array}{c}\text { Spain } \\
\text { Median, IQR }\end{array}$ & $\begin{array}{c}\text { Combined } \\
\text { Median, IQR }^{*}\end{array}$ \\
\hline Number of participants & 6 & 9 & 16 \\
Age (years) & $53[48 ; 58.5]$ & $56[52 ; 67]$ & $76[48.5 ; 63.5]$ \\
Months after stroke & $32[20 ; 55]$ & $2 / 6$ & $7[6 ; 31.5]$ \\
Sex (female/male) & $3 / 3$ & $3[2 ; 7]$ & $5 / 9$ \\
NIHSS + & $4.5[2.5 ; 6]$ & $44[41 ; 49]$ & $4[2 ; 6]$ \\
BBS $\neq$ & $51.5[46.5 ; 53.5]$ & $75[74 ; 90]$ & $47[43 ; 53]$ \\
Barthel Index & $97.5[95 ; 100]$ & & $92.5[75 ; 95]$ \\
\hline
\end{tabular}

* Interquartile Range.

† National Institutes of Health Stroke Scale (0-42 points).

‡ Berg Balance Scale (0-56 points).

\section{Safety and system usage}

One patient decided to quit after the hospital training, because of lack of appropriate infrastructure at home and technical problems with the system. Therefore, the data from that participant was not included. 15 patients completed a minimum of 12 weeks of training. No adverse events (e.g., pain or injury) occurred. Furthermore, the clinicians perceived no risky or critical situations while the patients trained at home with the REWIRE system. Monitored data showed that patients, trained below level 3 most of the time ( $>75 \%$ of the training sessions). The overall maximum monitoring score did not exceed 3.04 points: a score close to level 3 was observed 29 out of 900 times during training sessions. In none of the patients the system interrupted the therapy for security reasons (monitoring level 4 or above, Figure 7.3A).

On average, patients excluded 71\% (range 39\% and 92\%) of the scheduled sessions (Figure 7.3B). One participant had to travel abroad for work and interrupted training for 3 weeks. Other reasons for low compliance were technical issues or the requirement of more support from a clinican.

Patients used the REWIRE system on $3.58 \pm 1.59$ (Switzerland $3.63 \pm 1.87$, Spain 3.55 \pm 1.38 ) days per week. Sessions were scheduled on $5.11 \pm 1.63$ (Switzerland $6.22 \pm 1.6$, Spain $4.38 \pm 1.19$ ) days per week. The average training duration per week was $99 \pm 53 \mathrm{~min}$ (Switzerland $88 \pm 54 \mathrm{~min} /$ Spain $107 \pm 50 \mathrm{~min}$ ) (Figure 7.4A). It did not change over the course of twelve weeks of training $(\mathrm{p}=0.5328)$. 
A

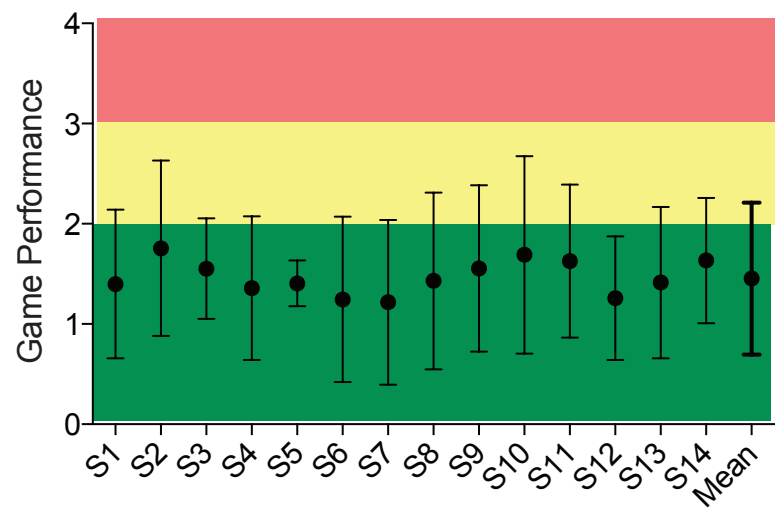

B

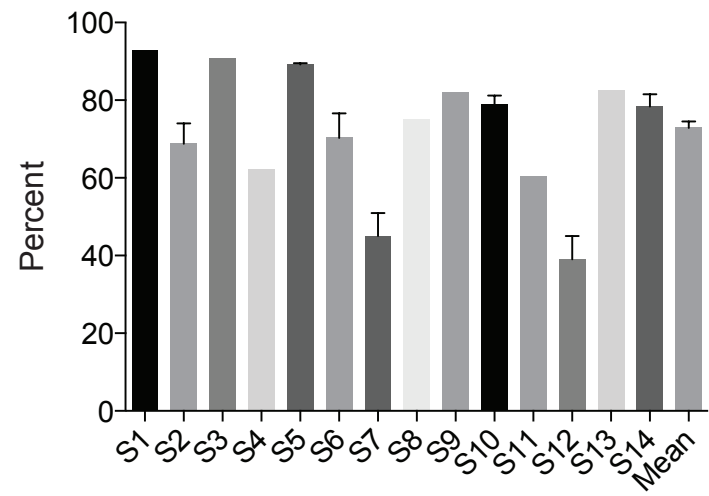

Figure 7.3: A) Game Performance. Mean and standard deviation of the monitoring level for each patient. 0-2 = green, no safety concern, 2-3 = yellow, the patient receives a warning, 3-4 = red, the exercise is interrupted and the virtual therapist shows the patient how to move correctly, $>4=$ application shuts down for severe safety concerns. B) Compliance in percentage. Percentage of executed relative to scheduled sessions.

The average training duration per training day was $28 \pm 15 \mathrm{~min}$ (Switzerland $24 \pm 11 \mathrm{~min}$ / Spain $30 \pm 7 \mathrm{~min}$ ) (Figure 7.4B). Six patients with high fall risk (BBS $\leq 45)$ 39,40 did use the system for similar amounts of time as compared with low risk subjects (BBS > 45) ( $\mathrm{p}$ $=0.533)$ (Figure 7.4C).

The TAM questionnaire showed high acceptance of the REWIRE system already at the beginning of the study (Table 7.2) and scores improved slightly but not significantly during the three months of training $(\mathrm{p}=0.197)$. All patients reported that they would have continued to use the REWIRE system after the trial if the system would have been made available to them. 
A

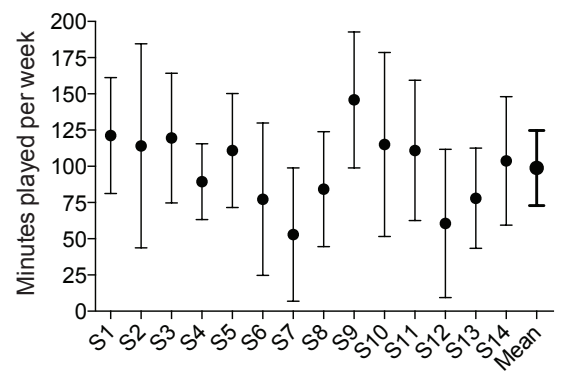

B

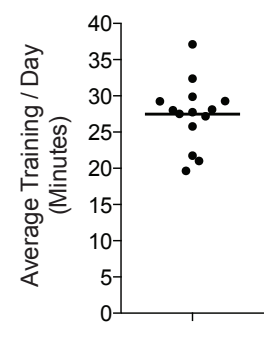

C

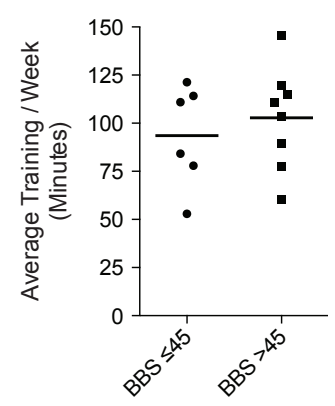

Figure 7.4: System usage.

A) Minutes trained per week per subject, mean and standard deviation B) Minutes trained per day C) Weekly training in patients with high versus low fall risk (BBS - Berg Balance Scale).

Table 7.2: Change in Technology Acceptance Model Questionnaire

\begin{tabular}{|c|c|c|c|c|c|c|}
\hline \multirow[b]{2}{*}{ Domain } & \multicolumn{2}{|c|}{$\begin{array}{c}\text { Swiss } \\
\text { Mean } \pm \text { Standard } \\
\text { Deviation }\end{array}$} & \multicolumn{2}{|c|}{$\begin{array}{c}\text { Spain } \\
\text { Mean } \pm \text { Standard } \\
\text { Deviation }\end{array}$} & \multicolumn{2}{|c|}{$\begin{array}{c}\text { Combined } \\
\text { Mean } \pm \text { Standard } \\
\text { Deviation }\end{array}$} \\
\hline & $\begin{array}{l}\text { Preinter- } \\
\text { vention }\end{array}$ & $\begin{array}{l}\text { Postinter- } \\
\text { vention }\end{array}$ & $\begin{array}{l}\text { Preinter- } \\
\text { vention }\end{array}$ & $\begin{array}{l}\text { Postinter- } \\
\text { vention }\end{array}$ & $\begin{array}{l}\text { Preinter- } \\
\text { vention }\end{array}$ & $\begin{array}{l}\text { Postinter- } \\
\text { vention }\end{array}$ \\
\hline $\begin{array}{l}\text { Perceived Ease } \\
\text { of Use }\end{array}$ & $5.5 \pm 1.58$ & $6.11 \pm 1.37$ & $6.04 \pm 1.21$ & $6.09 \pm 1.28$ & $5.8 \pm 1.17$ & $6.11 \pm 0.96$ \\
\hline $\begin{array}{l}\text { Perceived } \\
\text { Usefulness }\end{array}$ & $5.94 \pm 1.25$ & $6.67 \pm 0.66$ & $5.91 \pm 1.01$ & $5.91 \pm 1.17$ & $5.93 \pm 0.92$ & $6.24 \pm 0.85$ \\
\hline $\begin{array}{l}\text { Attitude } \\
\text { Toward Using }\end{array}$ & $4.33 \pm 2.79$ & $4.43 \pm 2.74$ & $6.33 \pm 0.8$ & $6.38 \pm 0.87$ & $5.51 \pm 0.92$ & $5.54 \pm 1.06$ \\
\hline $\begin{array}{l}\text { Behavioral } \\
\text { Intention to } \\
\text { Use }\end{array}$ & $6.11 \pm 1.47$ & $6.36 \pm 1.36$ & $5.7 \pm 1.33$ & $5.5 \pm 1.8$ & $5.89 \pm 0.88$ & $5.89 \pm 1.19$ \\
\hline TAM total & $5.54 \pm 1.91$ & $5.96 \pm 1.83$ & $5.98 \pm 1.13$ & $5.96 \pm 1.36$ & $5.8 \pm 0.68$ & $5.97 \pm 0.73$ \\
\hline
\end{tabular}

7-point Likert-scale: 1 = "I do not agree at all" to 7 = "I agree entirely".

\section{DISCUSSION}

This pilot study demonstrates that the REWIRE autonomous telerehabilitation system to train balance and gait is feasible and safe for stroke patients who live in their home and are able to walk independently. Safety is a major concern when training at home, as the patient is not supervised by a therapist in a one-to-one setting. Harmful movements could induce or aggravate high tone for patients with paresis or overload joints and induce pain. ${ }^{19,25}$ Falls are of concern during balance training. The REWIRE platform therefore 
continuously monitored the patient movement using an intelligent engine working on the data from the 3D camera and a pressure board.

The early warning to the patient, provided when slight deviations from correct movements were occurring (yellow or orange color) prevented more dangerous movements that would harm the patient. This preventive measure enabled a therapy that in no case had to be interrupted for security concerns nor led to serious adverse events. These results are in contrast to previous balance training studies, ${ }^{19,25}$ with related adverse events occurring. The system allowed movement pattern variability among subjects, as a certain movement may be harmful to some but not for other patients and the possibility to regulate and program them was rated as an important feature by all clinician.

Adequately setting the monitors was made possible because the therapist could evaluate the exercise safety while the patient performed supervised training in the clinic. The therapist then defined individual movement parameters and associated monitoring levels.

The compliance with the system was good. The average duration of 99 minutes per week, with training performed on 3.6 days/week is promising. This result compares well with other studies of home-based rehabilitation, in which duration of $105 \mathrm{~min} /$ week $^{41}$ and 85 $\mathrm{min} /$ week $^{42}$ were reported. Patients who used the REWIRE system less had a higher risk of falling, which could be due to fear of falling when using the system unassisted.

Patients used the REWIRE system at the hospital, before starting autonomous training at home. They were satisfied and motivated in using the system. Only a slight change in TAM was observed over the 3-months training period: TAM had possibly reached an already high value at the beginning of training. While high acceptance may be the result of a selection bias with patients open to technology being more likely to consent to the trial, acceptance may have been positively influenced by designing the REWIRE system intuitively to be used via gestures avoiding keyboard and mouse.

The REWIRE system provides valid scenarios that stimulate movement and behaviors that are relevant for daily life. This is implemented within a safe environment, which can be shaped in accordance with individual requirements and levels of ability. The responsive virtual environments allow patients to explore independently, increasing their sense of autonomy and independence in directing their own therapeutic experience. The controllability of the virtual environment allows for consistency in the way therapeutic protocols are delivered and performance is recorded, enabling an accurate valuation of a patient's performance over time. 


\section{CONCLUSIONS}

Autonomous telerehabilitation for balance and gait training with the REWIRE system is safe, feasible and can enable intensive rehabilitative therapy at home. Patients were satisfied with and motivated in using the system. The efficacy of the REWIRE therapy with respect to improving balance and reducing impairments should be investigated in future randomized trials.

\section{Declarations}

\section{Acknowledgements}

The authors would like to thank Dr. Viktoria Kluckner, Joachim Cerny and Elisabeth Sandner for their support with the study.

\section{Availability of data and supporting materials}

The data supporting the conclusions of this article are included within the article. Appendix A - Questionnaire. Technology Acceptance Model

\section{Consent for publication}

The individual in Figure 7.2 consented to the publication of the photograph.

\section{Ethics approval and consent to participate}

The study followed GCP-guidelines and was approved by the local Cantonal ethics committee "Nordwest- und Zentralschweiz" (EKNZ: 2014-061) and by the ethical committee from Virgen del Rocío Hospital in Seville, Spain (CEI code 2012PI/216). All subjects gave written informed consent in accordance with the declaration of Helsinki.

\section{REFERENCES}

1. Kwakkel G. Impact of intensity of practice after stroke: issues for consideration. Disability and Rehabilitation. 2006;28(13-14):823-830.

2. Stroke Unit Trialists C. Organised inpatient (stroke unit) care for stroke. Cochrane Database Syst Rev. 2013(9):CD000197.

3. Langhorne P, Coupar F, Pollock A. Motor recovery after stroke: a systematic review. Lancet Neurol. 2009;8(8):741-754.

4. Langhorne P, Duncan P. Does the organization of postacute stroke care really matter? Stroke. 2001;32(1):268-274.

5. Foley NC, Teasell RW, Bhogal SK, Doherty T, Speechley MR. The efficacy of stroke rehabilitation: a qualitative review. Topics in Stroke Rehabilitation. 2003;10(2):1-18. 
6. Dhurjaty S. The economics of telerehabilitation. Telemedicine journal and e-Health. 2004;10(2): 196-199.

7. Kwakkel G, Veerbeek JM, van Wegen EE, Wolf SL. Constraint-induced movement therapy after stroke. Lancet Neurol. 2015;14(2):224-234.

8. Cikajlo I, Rudolf M, Goljar N, Burger H, Matjacic Z. Telerehabilitation using virtual reality task can improve balance in patients with stroke. Disability and Rehabilitation. 2012;34(1):13-18.

9. Dellifraine JL, Dansky KH. Home-based telehealth: a review and meta-analysis. Journal of Telemedicine and Telecare. 2008;14(2):62-66.

10. Lohse KR, Hilderman CG, Cheung KL, Tatla S, Van der Loos HF. Virtual reality therapy for adults post-stroke: a systematic review and meta-analysis exploring virtual environments and commercial games in therapy. PLoS One. 2014;9(3):e93318.

11. Borghese NA, Murray D, Paraschiv-Ionescu A, de Bruin ED, Bulgheroni M, Steblin A, Luft A, et al. Rehabilitation at Home: A Comprehensive Technological Approach. In: Ma M, Jain CL, Anderson P, eds. Virtual, Augmented Reality and Serious Games for Healthcare 1. Berlin, Heidelberg: Springer Berlin Heidelberg; 2014:289-319.

12. Laver KE, Schoene D, Crotty M, George S, Lannin NA, Sherrington C. Telerehabilitation services for stroke. Cochrane Database of Systematic Reviews. 2013;12:CD010255.

13. Chen J, Jin W, Zhang XX, Xu W, Liu XN, Ren CC. Telerehabilitation approaches for stroke patients: Systematic review and meta-analysis of randomized controlled trials. Journal of Stroke \& Cerebrovascular Diseases. 2015;24(12):2660-2668.

14. Clark AM. Home based cardiac rehabilitation. British Medical Journal. 2010;340:b5510.

15. Clark AM, Munday C, McLaughlin D, Catto S, McLaren A, Macintyre PD. Peer support to promote physical activity after completion of centre-based cardiac rehabilitation: evaluation of access and effects. European Journal of Cardiovascular Nursing. 2012;11(4):388-395.

16. Laver KE, George S, Thomas S, Deutsch JE, Crotty M. Virtual reality for stroke rehabilitation. Cochrane Database Syst Rev. 2015;2:CD008349.

17. Llorens R, Noe E, Colomer C, Alcaniz M. Effectiveness, usability, and cost-benefit of a virtual reality-based telerehabilitation program for balance recovery after stroke: a randomized controlled trial. Archives of Physical Medicine and Rehabilitation. 2015;96(3):418-425 e412.

18. Corbetta D, Imeri F, Gatti R. Rehabilitation that incorporates virtual reality is more effective than standard rehabilitation for improving walking speed, balance and mobility after stroke: a systematic review. Journal of Physiotherapy. 2015;61(3):117-124.

19. Prosperini L, Fortuna D, Gianni C, Leonardi L, Marchetti MR, Pozzilli C. Home-based balance training using the Wii balance board: a randomized, crossover pilot study in multiple sclerosis. Neurorehabil Neural Repair. 2013;27(6):516-525.

20. Pirovano M, Mainetti R, Baud-Bovy G, Lanzi PL, Borghese NA. Intelligent Game Engine for Rehabilitation (IGER). IEEE Transactions on Computational Intelligence and Ai in Games. 2016;8(1):43-55.

21. Pirovano M, Mainetti R, Lanzi P, Borghese N. Game Engines and Exergames to Guide Rehabilitation at Home. In: Jensen W, Andersen OK, Akay M, eds. Replace, Repair, Restore, Relieve - Bridging Clinical and Engineering Solutions in Neurorehabilitation. Vol 7: Springer International Publishing; 2014:129-134.

22. Borghese NA, Pirovano M, Lanzi PL, Wuest S, de Bruin ED. Computational intelligence and game design for effective at-home stroke rehabilitation. Games for Health Journal. 2013;2(2): 81-88.

23. Microsoft. Microsoft Kinect. https://dev.windows.com/en-us/kinect, 2016.

24. Tyromotion. Tyromtion Tymo. 2016:http://tyromotion.com/produkte/tymo.

25. Bower KJ, Clark RA, McGinley JL, Martin CL, Miller KJ. Clinical feasibility of the Nintendo Wii for balance training post-stroke: a phase II randomized controlled trial in an inpatient setting. Clinical Rehabilitation. 2014;28(9):912-923. 
26. Fasola J, Matarić MJ. Socially assistive robot exercise coach: Motivating older adults to engage in physical exercise. In: Desai PJ, Dudek G, Khatib O, Kumar V, eds. Experimental Robotics: The 13th International Symposium on Experimental Robotics. Heidelberg: Springer International Publishing; 2013:463-479.

27. Pirovano M, Mainetti R, Baud-Bovy G, Lanzi PL, Borghese NA. Self-adaptive games for rehabilitation at home. Paper presented at: Computational Intelligence and Games (CIG), 2012 IEEE Conference on; 11-14 Sept. 2012.

28. Krakauer JW. Motor learning: its relevance to stroke recovery and neurorehabilitation. Current Opinion in Neurology. 2006;19(1):84-90.

29. Schollhorn WI, Beckmann H, Davids K. Exploiting system fluctuations. Differential training in physical prevention and rehabilitation programs for health and exercise. Medicina (Kaunas). 2010;46(6):365-373.

30. EU M, FDA, Health Canada, Swissmedic. International Conference on Harmonization Guideline for Good Clinical Practice - E6(R1) 1996.

31. Holden MK, Gill KM, Magliozzi MR. Gait assessment for neurologically impaired patients. Standards for outcome assessment. Physical Therapy. 1986;66(10):1530-1539.

32. Collen FM, Wade DT, Bradshaw CM. Mobility after stroke: reliability of measures of impairment and disability. International Disability Studies. 1990;12(1):6-9.

33. Berg K, Wood-Dauphinee S, Williams JI. The Balance Scale: reliability assessment with elderly residents and patients with an acute stroke. Scandinavian Journal of Rehabilitation Medicine. 1995;27(1):27-36.

34. Hu PJ, Chau PYK, Sheng ORL, Tam KY. Examining the technology acceptance model using physician acceptance of telemedicine technology. Journal of Management Information Systems. 1999;16(2):91-112.

35. Parra C, Jodar-Sanchez F, Jimenez-Hernandez MD, Vigil E, Palomino-Garcia A, MonicheAlvarez F, De la Torre-Laviana FJ, et al. Development, Implementation, and Evaluation of a Telemedicine Service for the Treatment of Acute Stroke Patients: TeleStroke. Interactive Journal of Medical Research. 2012;1(2):e15.

36. Jeon E, Park HA. Factors affecting acceptance of smartphone application for management of obesity. Healthcare Informatics Research. 2015;21(2):74-82.

37. Davis FD. Perceived usefulness, perceived ease of use, and user acceptance of information technology. Management Information Systems Quarterly. 1989;13(3):319-340.

38. King WR, He J. A meta-analysis of the technology acceptance model. Information \& Management. 2006;43(6):740-755.

39. Andersson AG, Kamwendo K, Seiger A, Appelros P. How to identify potential fallers in a stroke unit: validity indexes of 4 test methods. Journal of Rehabilitation Medicine. 2006;38(3):186-191.

40. Dogan A, Mengulluoglu M, Ozgirgin N. Evaluation of the effect of ankle-foot orthosis use on balance and mobility in hemiparetic stroke patients. Disability and Rehabilitation. 2011;33(1516):1433-1439.

41. Amirabdollahian F, Ates S, Basteris A, Cesario A, Buurke J, Hermens H, Hofs D, et al. Design, development and deployment of a hand/wrist exoskeleton for home-based rehabilitation after stroke-SCRIPT project. Robotica. 2014;32(08):1331-1346.

42. Sivan M, Gallagher J, Makower S, Keeling D, Bhakta B, O’Connor RJ, Levesley M. Home-based Computer Assisted Arm Rehabilitation (hCAAR) robotic device for upper limb exercise after stroke: results of a feasibility study in home setting. Journal of NeuroEngineering and Rehabilitation. 2014;11(1):163. 


\section{APPENDIX A \\ QUESTIONNAIRE TECHNOLOGY ACCEPTANCE MODEL}

\section{Perceived Ease of Use}

1. I found the exergames easy to use.

2. Learning to use the exergames would be easy for me.

3. My interaction with the exergames was clear and understandable.

4. I think the messages displayed by the REWIRE system would be clear.

5. I think it would be easy to acquire the skills required to use the exergames.

6. I found the lifestyle devices easy to use.

7. In general, I think the REWIRE system will be easy to use.

\section{Perceived Usefulness}

8. Using the exergames would enhance my effectiveness in training.

9. Using the exergames would improve my training performance.

10. Using the exergames would increase my productivity in training.

11. I found the exergames useful.

12. Using the lifestyle devices would improve the follow up of my rehabilitation.

13. In general, the REWIRE system may help to improve the rehabilitation.

\section{Attitude Toward Using}

14. I dislike the idea of using the exergames. (R)

15. I have a generally favorable attitude toward using exergames.

16. I believe it is (would be) a good idea to use these exergames for my training.

17. Using the exergames is a foolish idea. $(\mathrm{R})$

18. In general, I think that my family/friends would support the use of the REWIRE system.

\section{Behavioral Intention to Use}

19. I intend to use the exergames in my further training.

20. I will use the exergames often.

21. I intend to use the exergames frequently for my training.

22. I intend to use the lifestyle devices when it is necessary for my rehabilitation.

23. I intend to take part in the patients' community.

24. I intend to use the REWIRE system when it is available at my hours. 



\section{Chapter 8}

General discussion 


\section{STROKE REHABILITATION}

The field of stroke rehabilitation has changed in recent years with the development of advanced technologies to measure ${ }^{1,2}$ and treat ${ }^{3}$ recovery after a stroke. These technologies have introduced the way for a more personalised approach to the rehabilitation research process. ${ }^{4}$ However, these advanced technologies often lack context specificity, as the measurements and interventions are often performed in a rehabilitation clinic or private practice after rehabilitation discharge.

The present thesis focuses on two aspects of stroke rehabilitation: first, quantifying stroke patients' activities in the clinic and home environment during the rehabilitation process (Chapter 2) and secondly, evaluating sensor-based systems with a feedback modality for continued rehabilitation at home (Chapters 3-7).

In this general discussion, the context of each chapter is described, the findings are summarised and critically reviewed, and a future direction for sensor-based systems in the daily life of stroke rehabilitation is provided.

\section{EVALUATING A SENSOR-BASED SYSTEM TO MONITOR STROKE PATIENTS}

There is a growing interest in quantifying stroke patients' activities during the rehabilitation process to predict ${ }^{1}$ and sensitively measure motor recovery in stroke rehabilitation trials. ${ }^{5}$ For this reason, sensor-based systems have been developed to objectively measure outcomes. ${ }^{6,7}$ These systems have mainly been used for research in a laboratory setting but have the potential to measure functions and activities in daily life (ADL) situations. ${ }^{8}$ Information about the function and activities, measured with a sensor-based system, in daily life activities in patients after stroke has not been previously examined.

The sensor-based system developed in the European project, 'INTERACTION', and the metrics included from the on-body sensor data ${ }^{10}$ were applied while ischaemic stroke patients performed standard clinical assessments and measurements in a home-like environment. The sensor system is also able to capture movements while performing standard clinical assessments in the laboratory environment. ${ }^{11}$ In the prospective observational trial (Chapter 2), the sensor-based system was implemented in a clinical setting and a home environment to explore parallels between standard clinical assessments (i.e. capacity) and daily-life measures (i.e. performance). A research therapist monitored daily life measures to ensure the quality of the data. It was necessary to monitor the system because of sensor drift during 
the measurements, requiring a recalibration of the system. Sensor drift is the accumulating measurement error of acceleration sensors, gyroscopes, and magnetic-sensors over time. ${ }^{12}$ Positional tracking systems, such as cameras, and repeated calibrations can be employed to correct drift error continuously. Thus, the physical properties of the movements in the different stages of stroke rehabilitation were presented, and the use of the affected hand in daily life was compared to the measures from the clinical assessments. The measures derived from the movement sensor demonstrated that the metrics are likely to be more sensitive to change than clinical assessments. Furthermore, the motor function measured with clinical assessments, in which patients were encouraged by the therapist, did not reflect patients' behaviour concerning their impaired upper limb during ADLs. Stroke patients who score well in clinical assessments do not necessarily use their paretic arms in daily life. A limitation of this study is the small number of first-time ischaemic stroke patients included. Additionally, the early rehabilitation process was not observed. In future studies, first-time ischaemic stroke patients should not be the only patients monitored early after a stroke; haemorrhagic stroke patients and those with recurrent strokes should also be included. This is relevant because $20 \%$ of all stroke patients suffer a haemorrhagic stroke ${ }^{13}$ and approximately $25 \%$ suffer a recurrent stroke in the first 10 years. ${ }^{14}$ Future studies should investigate the natural course of upper limb movements not only in a clinical rehabilitation setting but also in daily life situations ${ }^{15}$ in order to determine the effectiveness of stroke rehabilitation interventions in the long term. ${ }^{2,16}$ Thereby, assessments with sensor-based systems are more sensitive to change, and rehabilitation interventions can be planned accordingly. Findings from these studies would then be generalisable to a larger portion of stroke survivors than those from prospective observational studies conducted that included only first-time ischaemic stroke patients. ${ }^{1}$ This knowledge could be used for designing rehabilitation trials, with a hetrogenous stroke population. Future studies should also observe the long-term effects of stroke rehabilitation intervention in the chronic stage, ${ }^{17}$ while measuring the daily life activities. The extent of upper extremity function and use during a patient's daily life activities is still unknown. Upper limb measures taken by sensor-based technologies will provide vital data by combining knowledge of clinical assessments and daily life. Moreover, these studies will not only examine the parallels between clinical assessments for upper limb function and activities as well as arm use in daily life but also provide insight into how upper limb use during daily life is measured using sensor-based technologies.

The sensor-based system described in Chapter 2 consists of 14 movement sensors placed in specific positions on the body and requires calibration and recalibration procedures during measurement. Furthermore, the transmission of data from the sensors to the receiver laptop and environmental factors, such as a change in floor levels, produced measurement errors. Therefore, the presence of a therapist was necessary. Together with the obtrusiveness 
of the system, this might influence the patients' performance during the measurements. A reduced sensor set would improve the problem of obtrusiveness, but would also imply that the measures from the sensor-based system will be less specific regarding movement quality because less information regarding the body segment orientation, relative segment position and joint angles is obtained. ${ }^{18}$ Another option to analyse movement quality, described in Chapter 5 is a reduced sensing system that uses a predefined condition for stroke rehabilitation intervention was presented. ${ }^{19,20}$ An automated, unsupervised, sensorbased assessment was added to the intervention. During the assessments, the patient was seated in front of a screen and performed standardised tasks. Stroke patients had to recalibrate the arm after every task at a predefined position. This offers the possibility to obtain objective measures of upper limb function.

Currently, in clinical stroke rehabilitation, assessments with sensor-based systems are possible but not yet integrated. To measure upper limb function during ADL a reduced sensor system should be used, noting the limitation of obtaining less advanced measures. ${ }^{21}$ To acquire exact upper limb movement parameters, such as kinematics, an extended sensorbased system can be used in the laboratory environment, ${ }^{11}$ or a therapist or technician, with an enormous effort, can constantly supervises the system during ADL measurements.

This knowledge can serve as a next step to developing patient-tailored rehabilitation.

\section{EVALUATING SENSOR-BASED SYSTEMS FOR STROKE REHABILITATION INTERVENTIONS}

The evidence for optimising post-stroke motor recovery supports high-level, intensive, specific, and enriched rehabilitation interventions in which patients receive feedback regarding their performance. ${ }^{16,22}$ However, these interventions can be difficult to deliver in daily practice because of high costs for healthcare providers. ${ }^{23,24}$ An option to increase the intensity of rehabilitation interventions is a programme in which patients independently perform motor rehabilitation in their home environment. The structure and format of the interventions can be separated in functional activities of daily life, by using tracking and feedback devices as well as structured exercises that use for example video games. Both interventions have the ability to enhance the use of the paretic side and increase patients' activity levels.

In order to meet these goals, a sensor-based system complemented with a vibrotactile actuator that can provide feedback based on users' performance was designed and tested. The aim was to coach stroke patients to use their affected upper extremity more in daily life 
(Chapter 3). The acceptance and usability of the device, 'Arm Usage Coach', was evaluated in a laboratory environment during a predefined daily task. The influence of the vibrotactile feedback modalities on arm use was also tested. Most patients reacted positively to the intuitive, vibrotactile feedback device and reported that the system could complement their current therapy. In the long term, sensor-based coaching systems could complement current therapy and be more context specific than standard therapy. Limitations of the system include its size and the requirement of a laptop to process movement data. To increase the usability of the sensor-based systems the size of the sensor should be reduced and the data should be processed on a portable smartphone. Furthermore, an integration of a combination of the vibrotactile and visual feedback and gamifications could increase the effectiveness of arm activity in daily life. With this multimodal approach, the efficacy of the system should be tested in a clinical trial.

The novel yband therapy system (yband therapy AG, Basel, Switzerland) has incorporated these contents, but the effects of such a sensor-based feedback system on upper limb use in daily life arm have not been investigated (Chapter 4). Enhancing functional activities during daily life is crucial, as interventions often lack context-specificity. This is because training is often performed in a clinic. To date, a beneficial effect of sensor-based feedback systems has not been reported from patients' paretic upper limb use in their daily lives, nor has an investigation of the influence of the feedback been completed. The protocol presented in Chapter 4 describes a randomised controlled trial that tests the efficacy of multimodal feedback to increase upper limb use in daily life as reported by chronic stroke patients who have completed their inpatient stroke rehabilitation. The multimodal feedback differentiates between extrinsic motivation from the wrist-worn device (e.g., vibrotactile sensation) and intrinsic motivation (e.g., use of the smartphone app). In addition, quantitative sensor data furnishes insight into the patients' compliance in completing arm activities while wearing the system. It is expected that the patient-reported amount of upper limb use in daily life in hemiparetic subjects will increase when wearing the wrist-worn, commercially available tracking device that provides multimodal feedback when compared with a control group. A positive result from this study would positively affect the development of sensor-based systems for rehabilitation of ADLs in patients' homes, beyond interventions in rehabilitation clinics. A similar approach has also been tested by Da-Silva et al., who demonstrated that home-based interventions can increase arm activities. ${ }^{25}$ As yet, it is not known how patients can be encouraged to keep using their paretic arm when they are in the home environemnt. The present study aims to address this issue in rehabilitation practice. The results can help therapists to remotely monitor patients and facilitate the patients to transfer learned patterns of arm movements from the therapy session to stroke patients' daily life. Based on this information, therapists can offer feedback and adapt therapy interventions 
accordingly. The proposed approach might also be beneficial for other diseases for which it is important to enhance arm use (e.g., multiple sclerosis).

Technologies for in-home interventions, including structured exercises, are being developed and evaluated to promote recovery without increasing demands on therapists' time. Two feasibility studies (Chapters 5 and 7) evaluated the use and safety of self-directed interventions in stroke patients' homes without the involvement of therapists. The level of compliance with using self-directed, sensor-based systems was high in the group, the members of which had mild-to-moderate upper limb and gait impairments. Patients with severe disabilities tended not to use these systems while performing exercises when they were not supported. In a limited number of moderately affected stroke patients, an increase in upper extremity function, measured with the Fugl-Meyer Assessment upper extremity (FMA-UE) and in arm workspace was observed. For upper limb activity measured with the Wolf Motor Function Test (WMFT), the training effects were minimal. This is likely due to the fact that there was no hand training integrated into the system. The new, commercially available, self-directed Armeo ${ }^{\circledR}$ Senso (Hocoma AG, Switzerland) has incorporated a hand module to obtain feedback and train grip function. The efficacy of this device requires investigation.

These findings are in line with a recent review that evaluated self-directed therapy programmes for upper extremities. ${ }^{26}$ It reports that these programmes have the potential to enhance arm recovery after a stroke. In addition, to increase the use of self-directed therapy systems by severely affected stroke patients, new devices such as exoskeletons could help compensate for the motor function of the hand during self-directed therapy and increase the use of the affected upper extremity. ${ }^{27,28}$ Future research using larger samples of patients including a control group should further examine the effects of treatment and identify those who would benefit most from self-directed, sensor-based systems at home. Additionally, the integrated in-system assessments, such as workspace and posture control assessments, to evaluate performance during exercises can prevent adverse events related to interventions. This is particularly important in interventions in which patients are encouraged to perform the exercise without a therapist's supervision.

Chapter 6 describes the protocol of the randomised controlled trial that investigates the effect of performance feedback and monetary rewards on movement performance using an adapted, sensor-based training system (Chapter 5). It is currently unknown how feedback and reward during rehabilitation interventions improve stroke patients' abilities and how this could change rehabilitation outcomes. It has been indicated that feedback and reward have a positive effect on motor learning. ${ }^{29,30}$ In addition, recent work supports the positive effects of monetary rewards on procedural learning (e.g., simple tasks) ${ }^{31}$ and skilled motor learning (e.g., 
complex motor tasks) ${ }^{32}$ as well as on motor adaption. ${ }^{33}$ Notably, all of these studies reported diverse effects of positive and negative rewards. Abe et al. and Galea et al., for example, found that a positive rewards influenced task consolidation and retention in healthy participants. With more knowledge regarding the type of feedback to provide to stroke patients during their rehabilitation, therapists and healthcare providers will be able to adapt therapy strategies to improve stroke patients' abilities. Reward, whether social or monetary, can be integrated into the rehabilitation programme, whether social or monetary. Technological interventions, such as sensor-based systems, can help to integrate more reward features.

\section{GENERAL CONCLUSION}

In recent years, sensor-based systems have become increasingly prevalent in measuring physical activity in stroke rehabilitation. They can also be applied in treatment by furnishing feedback on performance. ${ }^{34}$ The use of new rehabilitation technologies has made it possible to monitor the recovery of motor control in the first months after a stroke and to observe stroke patients' behaviour in their daily lives. ${ }^{35,36}$ Sensor-based systems enable performance monitoring for stroke patients. The feasibility of measuring the quality of movements with sensor-based systems has been presented in this thesis and has been revealed to be beneficial but challenging. Investigating the reliability and validity of these measures in large groups of patients and at predefined points of time after a stroke is vital to establish these measures as a standard for rehabilitation monitoring. Longitudinal studies can help to compare metrics of sensor-based systems with standard clinical scales. Based on these studies, standard measures can help to explain the natural course of post-stroke performance and can provide an early indication of functional deterioration.

For stroke neurorehabilitation interventions, sensor-based systems allow training in the patients' home environments thereby increasing the context-specificity of the therapy. Currently, evidence of how these systems can influence the use of the upper extremities or physical activity in people with stroke is limited. ${ }^{26,37}$ This is because to the fact that it is unknown how sensor-based systems can change behavioural strategies to improve physical activity for people after stroke. The development of sensor-based technologies for stroke patients will increase the acceptance for continued use, particular in persons who are less motivated, and are not included in clinical trials.

Research on using sensor-based systems for rehabilitation has only recently begun, but it can be expected that ongoing trials will identify the validity of the metrics derived from observational studies, and the efficacy on upper-extremity and physical activity outcomes from interventional trials. ${ }^{38,39}$ 


\section{OUTLOOK}

The sensor-based systems presented in this thesis ushered in the next step for individualised rehabilitation. The use of sensor-based systems is not limited to evaluating stroke survivors' capacity or performance and treating their disabilities. Other diseases influencing the function and activity of movements could profit from sensor-based systems. Improvements in sensor-based systems are required and should enhance the potential for implementation in stroke rehabilitation. For the assessment of motor performance and the execution of interventions in clinical rehabilitation, sensor-based systems need to fulfil different requirements. To assess the patients' disabilities with sensor-based systems, it is important that the system is unobtrusive and easily donned and doffed. The data analysis should be as straightforward as possible, and the metrics clinically relevant. This will increase the applicability in the daily clinical routine and the acceptance of therapists to use these systems. In addition, it is important to demonstrate to clinicians that the sensor-based systems are less time-consuming to use than standard clinical assessments. Hereby realistic, specific rehabilitation goals can be planned. Furthermore, the technical development of sensor-based systems to assess the patients' disability can also improve by adding additional measurement technologies, such as ultrasound ${ }^{40}$ or camera systems. ${ }^{41}$ The combination of Inertial Measurement Units (IMUs) and ultrasound sensors could facilitate the accurate evaluation of parameters, which depend on the relative position. ${ }^{40}$ The combination of IMUs, to track the motion of an object and the Simultaneous Localization and Mapping (SLAM) with camera systems to estimate objects in relation to the camera system in real-time. ${ }^{41}$ The combination of these approaches produces a vast amount of data, which requires adequate information technology for processing.

To treat patients' disabilities with sensor-based systems, it is essential that the systems are simple to use and low-cost and adapt to the changes in stroke subjects' performance during the interventions. A safety measure should be integrated to detect harmful movements and prevent a deterioration of movement performance. Furthermore, the possibility to telemonitor and adapt the intervention in the patients' homes and in daily life, according to the movement performance, will improve the usability and increase the effectiveness of the intervention. The task performed during the intervention should involve meaningful, challenging, and context-specific movement tasks. To avoid technical support during the intervention, the sensor-based devices should be extensively tested before delivery to stroke patients. This will help to increase the patients' acceptance of the system.

Sensor-based systems for rehabilitation interventions can integrate therapy into stroke patients' daily lives. This can prevent deterioration after discharge from rehabilitation 
centres. Nevertheless, the sensor-based methods that have been presented and tested in this thesis require further investigation in terms of efficacy and carryover of gains achieved in clinics to stroke patients' performance in daily life.

\section{REFERENCES}

1. Stinear CM, Barber PA, Petoe M, Anwar S, Byblow WD. The PREP algorithm predicts potential for upper limb recovery after stroke. Brain. 2012;135(8):2527-2535.

2. Cortes JC, Goldsmith J, Harran MD, Xu J, Kim N, Schambra HM, Luft AR, et al. A short and distinct time window for recovery of arm motor control early after stroke revealed with a global measure of trajectory kinematics. Neurorehabil Neural Repair. 2017;31(6):552-560.

3. Saposnik G, Mamdani M, Bayley M, Thorpe KE, Hall J, Cohen LG, Teasell R, et al. Effectiveness of Virtual Reality Exercises in STroke Rehabilitation (EVREST): rationale, design, and protocol of a pilot randomized clinical trial assessing the Wii gaming system. International Journal of Stroke. 2010;5(1):47-51.

4. Dobkin BH, Dorsch AK. The Evolution of Personalized Behavioral Intervention Technology: Will It Change How We Measure or Deliver Rehabilitation? Stroke. 2017;48(8):2329-2334.

5. Kwakkel G, Lannin NA, Borschmann K, English C, Ali M, Churilov L, Saposnik G, et al. Standardized measurement of sensorimotor recovery in stroke trials: Consensus-based core recommendations from the stroke recovery and rehabilitation roundtable. Neurorehabil Neural Repair. 2017;31(9):784-792.

6. Klaassen B, van Beijnum B-J, Weusthof M, Hofs D, van Meulen F, Droog E, Luinge H, et al. A full body sensing system for monitoring stroke patients in a home environment. In: Plantier G, Schultz T, Fred A, Gamboa H, eds. Biomedical Engineering Systems and Technologies: 7th International Joint Conference, BIOSTEC 2014, Angers, France, March 3-6, 2014, Revised Selected Papers. Cham: Springer International Publishing; 2015:378-393.

7. Leuenberger K, Gassert R. Low-power sensor module for long-term activity monitoring. Engineering in Medicine and Biology Society $(E M B C)$, Annual International Conference of the IEEE. 2011;2011:2237-2241.

8. Noorkoiv M, Rodgers H, Price CI. Accelerometer measurement of upper extremity movement after stroke: a systematic review of clinical studies. Journal of NeuroEngineering and Rehabilitation. 2014;11:144.

9. Klaassen B, van Beijnum BJ, Weusthof M, Hofs D, van Meulen F, Droog E, Luinge H, et al. A full body sensing system for monitoring stroke patients in a home environment. Biomedical Engineering Systems and Technologies, Biostec 2014. 2015;511:378-393.

10. van Meulen FB, Klaassen B, Held J, Reenalda J, Buurke JH, van Beijnum B-JF, Luft A, et al. Objective evaluation of the quality of movement in daily life after stroke. Frontiers in Bioengineering and Biotechnology. 2016;3(210).

11. van Meulen FB, Reenalda J, Buurke JH, Veltink PH. Assessment of daily-life reaching performance after stroke. Annals of Biomedical Engineering. 2015;43(2):478-486.

12. Luinge HJ, Veltink PH. Measuring orientation of human body segments using miniature gyroscopes and accelerometers. Medical \& Biological Engineering \& Computing. 2005;43(2):273282.

13. Feigin VL, Lawes CM, Bennett DA, Anderson CS. Stroke epidemiology: a review of populationbased studies of incidence, prevalence, and case-fatality in the late 20th century. Lancet Neurology. 2003;2(1):43-53. 
14. Mohan KM, Crichton SL, Grieve AP, Rudd AG, Wolfe CD, Heuschmann PU. Frequency and predictors for the risk of stroke recurrence up to 10 years after stroke: the South London Stroke Register. Journal of Neurology, Neurosurgery, and Psychiatry. 2009;80(9):1012-1018.

15. Veerbeek JM. Predicting real world physical activity and upper limb use after stroke (RE-USE). NCT03522519 2018; https://clinicaltrials.gov/ct2/show/NCT03522519. Accessed 23.08.2018.

16. Veerbeek JM, van Wegen E, van Peppen R, van der Wees PJ, Hendriks E, Rietberg M, Kwakkel $\mathrm{G}$. What is the evidence for physical therapy poststroke? A systematic review and meta-analysis. PLoS One. 2014;9(2):e87987.

17. Langhorne P, Bernhardt J, Kwakkel G. Stroke rehabilitation. Lancet. 2011;377(9778):1693-1702.

18. Roetenberg D, Luinge H, Slycke P. Xsens MVN: full 6DOF human motion tracking using miniature inertial sensors. Xsens Motion Technologies BV, Tech. Rep. 2009.

19. Wittmann F, Lambercy O, Gonzenbach RR, van Raai MA, Hover R, Held J, Starkey ML, et al. Assessment-driven arm therapy at home using an IMU-based virtual reality system. Paper presented at: Rehabilitation Robotics (ICORR), 2015 IEEE International Conference on2015.

20. Wittmann F, Held JP, Lambercy O, Starkey ML, Curt A, Hover R, Gassert R, et al. Self-directed arm therapy at home after stroke with a sensor-based virtual reality training system. Journal of NeuroEngineering and Rehabilitation. 2016;13(1):75.

21. Leuenberger K, Gonzenbach R, Wachter S, Luft A, Gassert R. A method to qualitatively assess arm use in stroke survivors in the home environment. Medical \& Biological Engineering \& Computing. 2016:1-10.

22. Lang CE, Macdonald JR, Reisman DS, Boyd L, Jacobson Kimberley T, Schindler-Ivens SM, Hornby TG, et al. Observation of amounts of movement practice provided during stroke rehabilitation. Archives of Physical Medicine and Rehabilitation. 2009;90(10):1692-1698.

23. Clarke DJ, Burton LJ, Tyson SF, Rodgers H, Drummond A, Palmer R, Hoffman A, et al. Why do stroke survivors not receive recommended amounts of active therapy? Findings from the ReAcT study, a mixed-methods case-study evaluation in eight stroke units. Clinical Rehabilitation. 2018:269215518765329.

24. Bernhardt J, Chan J, Nicola I, Collier JM. Little therapy, little physical activity: rehabilitation within the first 14 days of organized stroke unit care. Journal of Rehabilitation Medicine. 2007;39(1):43-48.

25. Da-Silva RH, van Wijck F, Shaw L, Rodgers H, Balaam M, Brkic L, Ploetz T, et al. Prompting arm activity after stroke: A clinical proof of concept study of wrist-worn accelerometers with a vibrating alert function. Journal of Rehabilitation and Assistive Technologies Engineering. 2018;5:2055668318761524.

26. Da-Silva RH, Moore SA, Price CI. Self-directed therapy programmes for arm rehabilitation after stroke: a systematic review. Clinical Rehabilitation. 2018:269215518775170.

27. Nycz CJ, Bützer T, Lambercy O, Arata J, Fischer GS, Gassert R. Design and Characterization of a Lightweight and Fully Portable Remote Actuation System for Use With a Hand Exoskeleton. IEEE Robotics and Automation Letters. 2016;1(2):976-983.

28. Nijenhuis SM, Prange GB, Amirabdollahian F, Sale P, Infarinato F, Nasr N, Mountain G, et al. Feasibility study into self-administered training at home using an arm and hand device with motivational gaming environment in chronic stroke. Journal of Neuroengineering and Rehabilitation. 2015;12.

29. Galea JM, Mallia E, Rothwell J, Diedrichsen J. The dissociable effects of punishment and reward on motor learning. Nature Neuroscience. 2015;18:597.

30. Quattrocchi G, Greenwood R, Rothwell JC, Galea JM, Bestmann S. Reward and punishment enhance motor adaptation in stroke. Journal of Neurology, Neurosurgery \& Psychiatry. 2017;88(9):730-736.

31. Wachter T, Lungu OV, Liu T, Willingham DT, Ashe J. Differential effect of reward and punishment on procedural learning. Journal of Neuroscience. 2009;29(2):436-443. 
32. Abe M, Schambra H, Wassermann EM, Luckenbaugh D, Schweighofer N, Cohen LG. Reward improves long-term retention of a motor memory through induction of offline memory gains. Current Biology. 2011;21(7):557-562.

33. Galea JM, Mallia E, Rothwell J, Diedrichsen J. The dissociable effects of punishment and reward on motor learning. Nature Neuroscience. 2015;18(4):597-602.

34. Henriksen A, Haugen Mikalsen M, Woldaregay AZ, Muzny M, Hartvigsen G, Hopstock LA, Grimsgaard S. Using fitness trackers and smartwatches to measure physical activity in research: Analysis of consumer wrist-worn wearables. Journal of Medical Internet Research. 2018;20(3):e110.

35. Held JPO, Klaassen B, Eenhoorn A, van Beijnum B-JF, Buurke JH, Veltink PH, Luft AR. Inertial sensor measurements of upper-limb kinematics in stroke patients in clinic and home environment. Frontiers in Bioengineering and Biotechnology. 2018;6(27).

36. Fanchamps M, Regterschot R, Boers L, Selles R, Ribbers G, Stam H, Bussmann J. Recovery of upper limb use after stroke and its relationship with upper limb function. Annals of Physical and Rehabilitation Medicine. 2018;61:e192-e193.

37. Lynch EA, Jones TM, Simpson DB, Fini NA, Kuys SS, Borschmann K, Kramer S, et al. Activity monitors for increasing physical activity in adult stroke survivors. Cochrane Database Syst Rev. 2018;7:CD012543.

38. Heron N, Kee F, Mant J, Reilly PM, Cupples M, Tully M, Donnelly M. Stroke Prevention Rehabilitation Intervention Trial of Exercise (SPRITE) - a randomised feasibility study. $B M C$ Cardiovascular Disorders. 2017;17(1):290.

39. Held JPO, Luft AR, Veerbeek JM. Encouragement-induced real-world upper limb use after stroke by a tracking and feedback device: A study protocol for a multi-center, assessor-blinded, randomized controlled trial. Frontiers in Neurology. 2018;9(13).

40. van Meulen FB, Weenk D, Buurke JH, van Beijnum BJ, Veltink PH. Ambulatory assessment of walking balance after stroke using instrumented shoes. Journal of NeuroEngineering and Rehabilitation. 2016;13(1):48.

41. Neges M, Koch C, König M, Abramovici M. Combining visual natural markers and IMU for improved AR based indoor navigation. Advanced Engineering Informatics. 2017;31:18-31. 

Summary 
Stroke is the third cause of long-term disability worldwide and the most common cause of disablement. Common, persisting disabilities are upper and lower extremity deficits, cognitive dysfunction, incontinence, and speech difficulties. Around $80 \%$ of stroke patients experience a unilateral motor deficit, which limits functionality and engagement in social life. Stroke patients require assistance for various activities of daily living and receive rehabilitation services to treat these disabilities.

Stroke rehabilitation is complex because of the varieties of brain lesions and diversity of physical and psychological issues. To tackle the complexity of post-stroke characteristics, it is important to assess the patients, set realistic goals, execute interventions, and reassess patients' abilities.

Stroke patients are assessed in a laboratory environment, where patients are encouraged by the therapist to perform predefined tasks. To measure patients' actions in their daily lives, clinicians and researchers traditionally rely on semi-structured interviews. Sensorbased technologies have been developed to objectively measure daily life activities and to allow for the continuous monitoring of daily life performance. Currently, it is not known how patients' performance, objectively measured by sensor-based systems during daily life, match and complement standard clinical assessments.

Interventions in stroke rehabilitation are well organised in a clinical setting within the first weeks after a stroke. However, the interventions after discharge are also important to improve performance and prevent deterioration in functioning. As a first step to prevent deterioration, sensor-based devices combined with feedback modalities are developed to motivate stroke patients to use and train their affected extremities more in daily life.

The present thesis focuses on the application of sensor-based systems in stroke rehabilitation. The objectives of this thesis are: 1) to evaluate a sensor-based system that can quantify stroke patients' upper limb activities in the rehabilitation clinic and in their home environment (Chapter 2); and 2) to evaluate the usability and efficacy of sensorbased systems with feedback modalities for stroke rehabilitation interventions that can be used in the patients' home environment (Chapters 3-7).

To assess the stroke patients, a sensor system called 'INTERACTION' was developed to explore parallels between standard clinical assessments (i.e. capacity) and daily-life measures (i.e. performance) in an observational study (Chapter 2). The measurements were performed in the rehabilitation clinic and in the patients' home environment. Newly developed metrics for classifying the activity and quality of upper extremity movement were applied. Their arm motor function, measured with standard clinical assessments, improved during the inpatient rehabilitation but declined in the first four weeks after discharge. 
Despite this deterioration in the clinical assessments, patients increased the number of reaches they performed of the affected side during daily life in their home environment. The metrics derived from the sensor system are likely to be more sensitive to change than clinical assessments. Furthermore, this study revealed that the motor function measured with clinical assessments did not reflect patients' behaviour in relation to their impaired upper limb during activities of daily living. Stroke patients who score well in clinical assessments do not necessarily use their paretic arm in daily life.

For stroke rehabilitation intervention, a sensor-based system combined with vibrotactile feedback, the 'Arm Usage Coach', was tested with patients in a laboratory environment (Chapter 3). The system aimed to influence arm use by monitoring performance and providing real-time feedback. The acceptance and usability were evaluated. Most patients found the vibrotactile feedback intuitive, agreeable and reported that the system could complement their current therapy.

The findings of Chapter 3 led to the development of a new sensor-based feedback system, the yband therapy system (Yband Therapy AG, Basel, Switzerland), which is described in Chapter 4 and is now being tested in a randomised clinical trial. The system monitors arm use in daily life and coaches stroke patients to increase use of their paretic arm in daily life with an integrated feedback system. The ongoing randomised controlled trial aims to test the efficacy of the feedback system in stroke patients who have completed their clinical rehabilitation. Results of this study will positively influence the development of sensor-based feedback systems for rehabilitation of real-world arm use in patients' homes, beyond interventions in rehabilitation clinics.

Technologies for in-home interventions, including structured exercises, are being developed and evaluated to promote recovery without increasing demands on therapists' time. Two feasibility studies (Chapters 5 and 7), the 'ArmeoSenso' for the upper extremity and the 'REWIRE' for balance and gait, evaluated the use and safety of self-directed interventions in stroke patients' homes without supervision of a therapists. Compliance with using these self-directed, sensor-based systems was high in patients who had mild-to-moderate upper extremity and gait impairments. Patients with severe disabilities tended to not use these systems. In addition, there were no safety issues in using the self-directed, sensor-based systems.

To evaluate the efficacy of the performance feedback and reward provision, an adapted sensor-based 'ArmeoSenso' system is currently being investigated in a randomised controlled trial described in Chapter 6. The results of this trial could emphasise the role of reward in stroke rehabilitation. With this knowledge, therapists and healthcare providers will be able to adapt strategies to diminish stroke patients' disabilities. 
The use of new rehabilitation technologies, such as sensor-based systems, have made it possible to monitor motor function and observe stroke patients' behaviour in their daily lives. Furthermore, these technologies can help measure function and activities in rehabilitation trials and profile the patients' recovery. In addition, sensor-based systems for stroke rehabilitation interventions allow training in the patients' home environments, thereby increasing the specificity of the therapy. As such, they can facilitate the integration of therapy into stroke patients' daily lives.

Nevertheless, the presented and tested sensor-based methods need to be further investigated in terms of efficacy as well as the carryover of gains achieved in clinics for mildly to moderately affected stroke patients' performance in their daily lives. 

Samenvatting

(Summary in Dutch) 
Beroertes zijn wereldwijd de derde oorzaak van langdurige invaliditeit. Veelvoorkomende beperkingen zijn paresen van armen en benen, cognitieve stoornissen, incontinentie en problemen met spreken. Ongeveer $80 \%$ van de patiënten met een beroerte hebben een eenzijdige parese van arm en/of been. Dit beperkt niet alleen het uitvoeren van dagelijkse activiteiten zoals het wassen en aankleden, maar ook sociale interactie en maatschappelijke participatie. Patiënten met een beroerte nemen deel aan een revalidatieprogramma om deze beperkingen te reduceren.

Revalidatie na een beroerte is complex, omdat de locatie van het hersenletsel van patiënt tot patiënt verschilt en daarmee de fysieke en psychologische problemen die zij ervaren. Om deze complexe problemen aan te pakken, is het belangrijk de patiënten eerst te onderzoeken en op basis daarvan realistische revalidatiedoelen en een behandelplan op te stellen. Tijdens en na de behandelperiode worden de beperkingen van de patiënten opnieuw beoordeeld.

Patiënten met een beroerte worden normaliter in een kliniek onderzocht met behulp van standaard klinische tests. Hierbij worden ze door de therapeut aangemoedigd om voorgedefinieerde taken uit te voeren. Om inzicht te krijgen in wat patiënten buiten therapieën om of in de thuissituatie doen, maken clinici en onderzoekers gewoonlijk gebruik van semigestructureerde interviews. Deze hebben als nadeel dat ze subjectief zijn en hoge eisen stellen aan het begrip en de communicatieve vaardigheden van patiënten. Sensorgebaseerde technologieën zijn ontwikkeld om op objectieve wijze dat wat patiënten tijdens het dagelijks leven doen (d.w.z. prestaties) te meten. Daarnaast kunnen ze deze prestaties gedurende meerdere uren of dagen registreren. Momenteel is het niet bekend of de prestaties van patiënten die objectief worden gemeten via sensorgebaseerde systemen tijdens het dagelijks leven overeenstemmen met standaard klinische beoordelingen en hoe ze deze aanvullen.

Revalidatie-interventies na beroertes zijn goed georganiseerd gedurende opname in een ziekenhuis en revalidatiecentrum. Voor patiënten die niet volledig zijn hersteld is het echter belangrijk dat revalidatie na ontslag wordt voortgezet teneinde achteruitgang te voorkomen en zo mogelijk het functioneren verder te verbeteren. Sensorgebaseerde systemen gecombineerd met feedbackmodaliteiten spelen hierop in door patiënten na een beroerte te motiveren om bijvoorbeeld hun aangedane arm meer te gebruiken in het dagelijkse leven.

Het onderhavige proefschrift richt zich op het gebruik van sensorgebaseerde systemen in de beroerterevalidatie. De doelstellingen van dit proefschrift zijn: 1) het evalueren van een sensorgebaseerd systeem dat de activiteiten van de armen van patiënten met een beroerte kan meten in de revalidatiekliniek en in de thuisomgeving (Hoofdstuk 2); en 2) het evalueren van de bruikbaarheid en effectiviteit van sensorgebaseerde systemen met 
feedbackmodaliteiten voor revalidatie na een beroerte die gebruikt kunnen worden in de thuisomgeving (Hoofdstukken 3 tot en met 7).

In een observationele studie werden bij patiënten met een beroerte de parallelen tussen standaard klinische tests (d.w.z. capaciteit) en metingen tijdens het dagelijks leven (d.w.z. prestaties) onderzocht (Hoofdstuk 2). Hiervoor werd het 'INTERACTION' sensorsysteem gebruikt. De metingen werden uitgevoerd in de revalidatiekliniek en in de thuisomgeving van de patiënten. Er werden nieuw ontwikkelde parameters voor het classificeren van zowel de mate van armactiviteit als de kwaliteit van de armbeweging toegepast. De motorische functie van de aangedane arm, gemeten met standaard klinische tests, verbeterde tijdens klinische revalidatie, maar verslechterde tijdens de eerste vier weken na ontslag uit de kliniek. Ondanks deze achteruitgang verhoogden patiënten het aantal keren dat ze naar iets reikten in hun thuisomgeving. De uit het sensorsysteem afgeleide parameters zijn wellicht gevoeliger voor verandering dan standaard klinische tests. Bovendien onthulde deze studie dat de met klinische tests gemeten motorische functie het gebruik van de aangedane arm in het dagelijks leven niet altijd weerspiegelden: niet alle patiënten die goed scoorden op klinische tests gebruikten hun paretische arm in het dagelijks leven.

In Hoofdstuk 3 werd de 'Arm Usage Coach' in de kliniek getest op acceptatie en bruikbaarheid. Het systeem trachtte het armgebruik in het dagelijks leven te beïnvloeden door prestaties te controleren en realtime vibrotactiele feedback te geven. De meeste patiënten vonden de vibrotactiele feedback aangenaam en rapporteerden dat het systeem hun huidige therapie kon aanvullen.

De bevindingen van Hoofdstuk 3 leidden tot de ontwikkeling van een nieuw sensorgebaseerd feedbacksysteem (yband therapy system, Yband Therapy AG, Basel, Zwitserland) dat beschreven wordt in Hoofdstuk 4. Het systeem meet hoe vaak patiënten na een beroerte hun aangedane arm in het dagelijks leven bewegen. Vervolgens stimuleert het systeem middels een geïntegreerd feedbacksysteem om de prestaties te verhogen. Momenteel wordt de effectiviteit van dit systeem getest in een gerandomiseerde gecontroleerde studie waaraan 62 thuiswonende patiënten met een beroerte zullen deelnemen. Naar verwachting zullen de resultaten van deze studie de ontwikkeling van sensorgebaseerde feedbacksystemen voor het herstel van armgebruik in het dagelijks leven positief beïnvloeden.

Momenteel worden technologieën voor interventies in de thuisomgeving ontwikkeld en geëvalueerd om herstel na een beroerte te bevorderen zonder dat de inzet van therapeuten toeneemt. Twee haalbaarheidsstudies (Hoofdstukken 5 en 7), de 'ArmeoSenso' voor de aangedane arm en het REWIRE-therapiesysteem voor de balans en het gaan, evalueerden het gebruik en de veiligheid van ongesuperviseerd oefenen door patiënten met een beroerte 
in hun thuisomgeving. De therapietrouw was hoog bij patiënten die milde tot matige beperkingen hadden. Patiënten met zeer ernstige beperkingen waren niet snel geneigd deze systemen te gebruiken.

Om de effectiviteit van de geleverde prestatiefeedback en beloningen te evalueren, wordt momenteel een aangepast sensorgebaseerd 'ArmeoSenso'-systeem in een gerandomiseerde studie onderzocht (Hoofdstuk 6). De resultaten zouden het belang van beloningen bij revalidatietraining kunnen onderstrepen. Met deze kennis zullen therapeuten en andere zorgverleners hun behandelstrategieën kunnen aanpassen met als doel beperkingen van patiënten met een beroerte verder te verminderen.

Het gebruik van nieuwe technologieën zoals sensorgebaseerde systemen heeft het mogelijk gemaakt de motorische functie te monitoren en de prestaties van patiënten met een beroerte in hun dagelijks leven objectief vast te leggen. Sensorgebaseerde systemen maken het mogelijk dat patiënten in hun thuisomgeving trainen, waardoor de specificiteit van de therapie wordt verhoogd. Patiënten gebruiken deze sensorgebaseerde systemen ook daadwerkelijk en de training is veilig gebleken.

Door de ontwikkeling van nieuwe revalidatietechnologieën kunnen de motorische functie en de prestaties van patiënten met een beroerte worden geobjectiveerd. Tevens kunnen ze worden ingezet om patiënten die mild tot matig aangedaan zijn in staat te stellen in de thuissituatie te oefenen. Daarmee kunnen ze de integratie van de therapie in het dagelijkse leven van patiënten vergemakkelijken. Ondanks dat de eerste resultaten veelbelovend zijn, moeten de gepresenteerde sensorgebaseerde systemen verder onderzocht worden op hun effectiviteit, inclusief de transfer van de in de kliniek bereikte resultaten naar het dagelijks leven. 

Zusammenfassung

(Summary in German) 
Schlaganfall ist weltweit die dritthäufigste Ursache für dauerhafte Einschränkungen und die häufigste Ursache für Behinderungen. Häufige Einschränkungen sind Defizite der oberen und unteren Extremitäten, kognitive Dysfunktion, Inkontinenz und Sprachstörungen. Etwa 80\% der Schlaganfallpatienten leiden unter einem einseitigen motorischen Defizit, das die Funktionalität und die Teilnahme am gesellschaftlichen Leben einschränkt. Schlaganfallpatienten benötigen Unterstützung bei verschiedenen Alltagsaktivitäten und erhalten Rehabilitation zur Behandlung dieser Einschränkungen.

Die Rehabilitation nach Schlaganfall ist aufgrund der unterschiedlichen Hirnläsionen und der Vielfalt der physischen und psychischen Probleme komplex. Um die vielfältigen Eigenschaften nach einem Schlaganfall zu behandeln, ist es wichtig, die Patienten zu untersuchen, realistische Ziele festzulegen, Therapien durchzuführen und im Anschluss daran die Einschränkungen der Patienten neu zu bewerten.

Schlaganfallpatienten werden unter Laborbedingungen beurteilt, wobei sie vom Therapeuten aufgefordert werden bestimmte Aufgaben durchzuführen. Um den Alltag der Patienten zu messen, sind Therapeuten, Ärzte und Wissenschaftler üblicherweise auf teilstrukturierte Interviews angewiesen. Um die Aktivitäten der Patienten im Alltag objektiv und kontinuierlich zu messen, wurden sensorbasierte Technologien entwickelt. Derzeit ist nicht bekannt, wie die tatsächlichen Aktivitäten und Leistungen der Patienten im Alltag objektiv durch sensorbasierte Technologien gemessen werden können und wie diese mit den klinischen Tests übereinstimmen, bzw. welche ergänzenden Informationen die neuen Technologien liefern können.

Die Therapien in der Rehabilitation sind in den ersten Wochen nach dem Schlaganfall im Krankenhaus und in den Rehabilitationskliniken gut organisiert. Um die Leistungen der Patienten auch nach der Entlassung aus den Kliniken zu verbessern und einer Verschlechterung vorzubeugen, ist es wichtig, Therapien für den Alltag der Patienten zu planen. Um Schlaganfallpatienten zu motivieren, die betroffenen Extremitäten im Alltag einzusetzen und zu trainieren wurden sensorbasierte Technologien und Feedbacksysteme kombiniert.

Die vorliegende Doktorarbeit fokussiert sich auf die Anwendung sensorbasierter Systeme in der Schlaganfallrehabilitation. Die Ziele dieser Dissertation sind: 1) Bewertung eines sensorbasierten Systems, das die Aktivitäten der oberen Extremitäten von Schlaganfallpatienten in der Rehabilitationsklinik und bei den Patienten zu Hause quantifizieren kann (Kapitel 2); und 2) Die Benutzerfreundlichkeit und Effektivität von sensorbasierten Feedbacksystemen für Rehabilitationsinterventionen im Alltag von Schlaganfallpatienten zu bewerten (Kapitel 3-7). 
Um die Aktivitäten von Schlaganfallpatienten zu messen, wurde das Sensorsystem „INTERACTION“ entwickelt und in einer Beobachtungsstudie (Kapitel 2) verwendet, um den Zusammenhang zwischen klinischen Tests (d.h. Fähigkeit) und Alltag (Performance) zu untersuchen. Die Messungen mit dem Sensorensystem wurden in der Rehabilitationsklinik und bei den Patienten zu Hause durchgeführt. Um die Aktivität und die Qualität der Bewegungen der oberen Extremität zu klassifizieren, wurden neu entwickelte Metriken verwendet. Die mit klinischen Tests gemessene Armfunktion verbesserte sich während der stationären Rehabilitation, verschlechterte sich aber in den ersten vier Wochen nach der Entlassung. Trotz der Verschlechterung erhöhten die Patienten die Anzahl Greifbewegungen im Alltag. Die aus dem Sensorensystem abgeleiteten Metriken reagieren vermutlich empfindlicher auf Veränderungen als klinische Tests. Darüber hinaus zeigte diese Studie, dass das mit klinischen Tests gemessene Bewegungsverhalten der Patienten, bezogen auf die betroffene obere Extremität, nicht die Aktivitäten im Alltag widerspiegeln. Schlaganfallpatienten, die bei klinischen Tests gut bewertet werden, verwenden ihre paretischen Arme nicht unbedingt im täglichen Leben.

Für die Therapien in der Schlaganfallrehabilitation wurde der „Arm Usage Coach”, ein sensorbasiertes System kombiniert mit Vibrations-Feedback, an Patienten unter Laborbedingungen getestet (Kapitel 3). Das Ziel des Systems ist, die Armnutzung zu beeinflussen, indem es das Bewegungsverhalten misst und Echtzeit-Feedback liefert. Die Akzeptanz und Benutzerfreundlichkeit wurden bewertet. Die meisten Patienten fanden das intuitive Vibrations-Feedback angenehm und gaben an, dass das System ihre aktuelle Therapie ergänzen könnte.

Die Erkenntnisse aus Kapitel 3 führten zur Entwicklung eines neuen sensorbasierten Feedbacksystems, das yband therapy system (Yband Therapy AG, Basel, Schweiz), das in Kapitel 4 beschrieben wird, und derzeit in einer randomisierten klinischen Studie getestet wird. Das System überwacht Alltagsaktivitäten und trainiert Schlaganfallpatienten mit einem integrierten Feedbacksystem. Die randomisierte kontrollierte Studie wird die Wirksamkeit des Feedbacksystems an Schlaganfallpatienten testen, welche die Rehabilitation abgeschlossen haben, um die Verwendung der oberen Extremitäten zu erhöhen. Die Ergebnisse dieser Studie werden sich positiv auf die Entwicklung sensorbasierter Feedbacksysteme zur Rehabilitation und der tatsächlichen Nutzung des Arms im Alltag beim Patienten zu Hause, über die Therapie der Rehabilitationskliniken hinaus, auswirken.

Um die Erholung nach Schlaganfall zu unterstützen, wurden Technologien für die Therapie zu Hause entwickelt und untersucht. Diese schließen strukturierte Übungen ein, die den Zeitaufwand für Therapeuten nicht erhöhen. Zwei Machbarkeitsstudien (Kapitel 5 und 7), die „ArmeoSenso“ Studie für die oberen Extremitäten und die REWIRE-Studie für 
Gleichgewicht und Gang, evaluierten die Verwendung und die Sicherheit von selbstbestimmten Interventionen bei Schlaganfallpatienten zu Hause, ohne eine Betreuung durch einen Therapeuten. Die Schlaganfallpatienten mit leichten bis mittelschweren Beeinträchtigungen der oberen Extremität und des Ganges verwendeten Therapie Systems häufiger. Patienten mit schweren Beeinträchtigungen neigten dazu die Systems nicht zu verwenden. Zudem konnten während der Verwendung von selbstbestimmten Sensorensystemen, für die Therapie keine unerwünschte Ereignisse festgestellt werden.

Um die Wirksamkeit von Leistungs-Feedbacks und Belohnungen zu evaluieren, wird derzeit eine randomisierte kontrollierte Studie (beschrieben in Kapitel 6) durchgeführt. Hierbei wird ein überarbeitetes sensorbasiertes „ArmeoSenso“-System verwendet. Die Ergebnisse dieser Studie könnten die Bedeutung von Belohnungen im Rehabilitationstraining hervorheben. Mit diesen Kenntnissen werden Therapeuten und die im Gesundheitswesen tätigen Personen in der Lage sein, Therapien anzupassen, um die Einschränkungen von Schlaganfallpatienten zu verringern.

Durch den Einsatz neuer Rehabilitationstechnologien, wie beispielsweise sensorbasierte Systeme, ist es möglich geworden, die Motorik von Schlaganfallpatienten zu messen und zu beobachten wie sie sich im Alltag tatsächlich verhalten. Des Weiteren bieten sensorbasierte System die Möglichkeit, die Einschränkungen im Alltag des Patienten in Rehabilitationsstudien zu messen und den Verlauf der Erholung darzustellen. Zudem ermöglichen sensorbasierte Systeme für die Schlaganfallrehabilitation das Training beim Patienten zu Hause und erhöhen damit die Spezifität der Therapie. Als solches unterstützen sie die Integration von der Therapie in das alltägliche Leben.

Dennoch sollten die beschriebenen und getesteten sensorbasierten Methoden hinsichtlich ihrer Wirksamkeit weiter untersucht werden. Ausserdem sollte der Transfer der erworbenen Fähigkeit, von der stationären Klinik in den Alltag, bei leicht bis mittelschwer betroffenen Schlaganfallpatienten weiter untersucht werden. 
- "Drift" can be a disadvantage of sensor-based technologies, an everincreasing difference between where a sensor thinks an object is and the actual position. It can also happen to anyone during everyday life and it is important that systems and persons recalibrate. - 
Acknowledgements 
First, I would also like to thank Prof. Dr. Ir. Peter Veltink for agreeing to be the head of my $\mathrm{PhD}$ committee and for giving me the freedom to perform my research outside the University of Twente. I have been impressed by his drive to bridge the gap between clinicians and engineers and implement hypothesis-driven sensor-based applications in neurorehabilitation research.

I also want to express my gratitude to Prof. Dr. Med. Andreas Luft for giving me the opportunity, to collaborate with all the partners within my $\mathrm{PhD}$, to work in this interesting research field of stroke rehabilitation and to conduct this thesis at the University of Zurich. His enormous scientific knowledge and experience were highly influential for my development as a researcher.

Moreover, I would like to thank Prof. Dr. Jaap Buurke, my co-promotor, for his clinical and scientific feedback and the inspiring discussions during the meetings at international conferences and the $\mathrm{PhD}$-progress meetings.

I would like to express my thankfulness for the great support from our research team of the University of Zurich and the University Twente.

A special thank goes to my teammates and friends:

I would also thank Bert Eenhoorn for his support during the last few years. He was always surprising me with new ideas and encouraged me to progress in the scientific work during our Wednesday evening-meetings. "Ich danke dir für die guten Gespräche und dein hilfreiches Feedback in allen Lebenslagen.«

Janne Veerbeek, who supported me in finalizing the $\mathrm{PhD}$ thesis. Here scientific knowledge in stroke rehabilitation and the critical is questioning of research projects is impressive. »Herzlichen Dank, für die guten Diskussionen über Projekte innerhalb und ausserhalb der Schlaganfall-Forschung.«

Jannie van Duinen, for your daily support to perform clinical trials in our small E15environment. »Danke für deine Unterstützung im Beruflichen und insbesonder im Privaten.«

Mario Widmer, for the scientific exchange in planning and organizing clinical trials to evaluate performance feedback and reward.

Frieder Wittmann, for the discussion about sensor-based systems and the pitfalls of movement sensors. 
Bart Klaassen, for his technical support during the INTERACTION project and the discussion about physical fitness.

Thanks to all the research collaborators of the INTERACTION project, the REWIRE project, the ArmeoSenso project, and the ISEAR project.

Many thanks goes to all stroke patients for their time and enthusiasm during the studies of this thesis. Without their patience and their time, they brought along it would have been impossible to perform this studies.

I also would like to thank my parents (Anne and Lothar Held) for their support and their coaching in the last years, during my PhD. » Ihr habt mir geholfen eine andere Sichtweise auf meine Arbeit zu haben und die wichtigen Dinge im Leben nicht zu vernachlässigen. « Last but not least, I would like to express my deepest thankfulness to my wife Maike Held and my children (Jaron and Madita) for their unconditional love and support. »Ihr bringt mich immer wieder zurück in den Alltag und helft mir von der Wissenschaft weg $\mathrm{zu}$ "driften".»

- Thank you / Dank u wel / Herzlichen Dank - 

About the author 


\section{CURRICULUM VITAE}

Jeremia Held was born on July $10^{\text {th }}, 1981$ in Bielefeld, Germany. After primary school, he went to the Gesamtschule Quelle in Bielefeld and graduated (Abitur) in 2001. The following year he did mandatory community service at the physical therapy department of the General Hospital Bielefeld with a subsequent one-year world cycling trip. In 2003, he started studying physical education and mathematics at the University of Bielefeld. One year later, he switched to physical therapy at the Helmut Rödler Schule Chemnitz to become a physiotherapist. After graduation in 2007, he worked as a therapist in different rehabilitation clinics and private practices

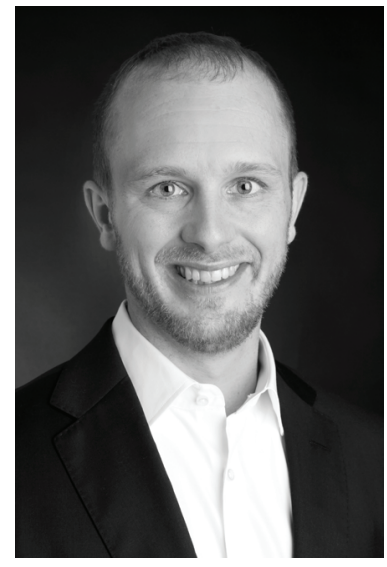
in Germany and Switzerland. During this time, he attended numerous advanced training courses (e.g., Manual Therapy, Bobath-concept) to expand his knowledge in treating patients and focused at the end in stroke rehabilitation. His drive to improve the evidence-base of physical therapy in neurorehabilitation, he started a master program in Neurorehabilitation science at the Danube University Krems, Austria, in 2011 and obtained his Master of Science degree in 2014. During the master program, he started working as research-physiotherapist in the group of Prof. Dr. med. Andreas Luft at the Division of Vascular Neurology and Neurorehabilitation, Department of Neurology, University of Zurich and University Hospital Zurich, Switzerland. Here, he was part of several national and international projects (e.g., INTERACTION, Rewire, ArmeoSenso) and got in contact with Prof. Dr. Ir. Peter Veltink of the University of Twente, Enschede, the Netherlands. This scientific environment motivated him to start the PhD-program at the University of Twente in 2015. He first worked on the development and evaluation of sensor-based systems for stroke rehabilitation, followed by the planning and organization stroke rehabilitation trials at the University of Zurich and University Hospital Zurich, Switzerland and the cereneo, center for neurology and rehabilitation. 


\section{BIBLIOGRAPHY}

\section{Peer-reviewed publications (Thesis)}

Held JPO, Klaassen B, Eenhoorn A, van Beijnum B-JF, Buurke JH, Veltink PH, Luft AR. Inertial sensor measurements of upper limb kinematics in stroke patients in clinic and home environment. Frontiers in Bioengineering and Biotechnology. 2018;6(27).

Held JPO, Luft AR, Veerbeek JM. Encouragement-induced real-world upper limb use after stroke by a tracking and feedback device: A study protocol for a multi-center, assessorblinded, randomized controlled trial. Frontiers in Neurology. 2018;9(13).

Held JP, Ferrer B, Mainetti R, Steblin A, Hertler B, Moreno-Conde A, Duenas A, Pajaro M, Parra-Calderón CL, Vargiu E, Zarco MJ, Barrera M, Echevarria C, Jodar-Sanchez F, Luft AR, Borghese NA. Autonomous rehabilitation at stroke patients home for balance and gait: safety, usability and compliance of a virtual reality system. European Journal of Physical and Rehabilitation Medicine. 2018;54(4):545-553.

Widmer M, Held JP, Wittmann F, Lambercy O, Lutz K, Luft AR. Does motivation matter in upper-limb rehabilitation after stroke? ArmeoSenso-Reward: study protocol for a randomized controlled trial. Trials. 2017;18(1):580.

Held JP, Klaassen B, van Beijnum B-JF, Luft AR, Veltink PH. Usability evaluation of a vibrotactile feedback system in stroke subjects. Frontiers in Bioengineering and Biotechnology. 2017;4(98).

Wittmann F, Held JP, Lambercy O, Starkey ML, Curt A, Hover R, Gassert R, Luft AR, Gonzenbach RR. Self-directed arm therapy at home after stroke with a sensor-based virtual reality training system. Journal of Neuroengineering and Rehabilitation. 2016;13(1):75.

\section{Peer-reviewed journal publications (0ther)}

Klaassen B, Van Beijnum BF, Held JPO, Reenalda J, Van Meulen FB, Veltink PH, Hermens HJ. Usability evaluations of a wearable inertial sensing system and quality of movement metrics for stroke survivors by care professionals. Frontiers in Bioengineering and Biotechnology. 2017;5:20.

Widmer M, Ziegler N, Held JPO, Luft A, Lutz K. Rewarding feedback promotes motor skill consolidation via striatal activity. Progress in Brain Research. 2016;229:303-323. 
Van Meulen FB, Klaassen B, Held JPO, Reenalda J, Buurke JH, Van Beijnum B-JF, Luft A, Veltink PH. Objective evaluation of the quality of movement in daily life after stroke. Frontiers in Bioengineering and Biotechnology. 2016;3(210).

Hofmann P, Held JPO, Gassert R, Lambercy O. Assessment of movement patterns in stroke patients: a case study with the Virtual Peg Insertion Test. Proceedings of the International Convention on Rehabilitation Engineering \& Assistive Technology; 2016.

Klaassen B, Van Beijnum B-J, Weusthof M, Hofs D, Van Meulen F, Droog E, Luinge H, Slot L, Tognetti A, Lorussi F, Paradiso R, Held JPO, Luft A, Reenalda J, Nikamp C, Buurke J, Hermens $\mathrm{H}$, Veltink P. A full body sensing system for monitoring stroke patients in a home environment. In: Plantier G, Schultz T, Fred A, Gamboa H, eds. Biomedical Engineering Systems and Technologies: 7th International Joint Conference, BIOSTEC 2014, Angers, France, March 3-6, 2014, Revised Selected Papers. Cham: Springer International Publishing; 2015:378-393.

\section{Peer-reviewed conference publications}

Held JPO, Veltink PH, Van Meulen FB, Luft AR, Buurke JH. Measurement of upper limb function during daily life after stroke. Paper presented at: International Conference on NeuroRehabilitation 2018.

Ryser F, Bützer T, Held JPO, Lambercy O, Gassert R. Fully embedded myoelectric control for a wearable robotic hand orthosis. Paper presented at: Rehabilitation Robotics (ICORR), 2017 International Conference on 2017.

Wittmann F, Lambercy O, Gonzenbach RR, Van Raai MA, Hover R, Held JPO, Starkey ML, Curt A, Luft A, Gassert R. Assessment-driven arm therapy at home using an IMUbased virtual reality system. Paper presented at: Rehabilitation Robotics (ICORR), 2015 IEEE International Conference on 2015.

\section{Patent}

Franinovic K, Meier D, Bauer S, Gassert R, Kim Y, Leuenberger K, Luft A, Held JPO. Detecting an evaluating movements of a user. Google Patents; 2017. WO 2017/032765. 2017. 


\section{Conference presentations}

Oral presentation:

$9^{\text {th }}$ Swiss Stroke Trialists' Meetings - 2019, Zurich, Switzerland

- Encouragement-induced real-world upper limb use after stroke by a tracking and feedback device

$2^{\text {nd }}$ Interprofessional Stroke Symposium for Nursing and Therapy - 2019, Zurich,

Switzerland

- Early mobilization and prediction

SGNR-Symposium für Interprofessionelle Neurorehabilitation - 2018, Zurich,

Switzerland

- Sensorbasierte Neurorehabilitation - Assessments und Therapie

International Conference on Neurorehabilitation - ICNR 2018, Pisa, Italy

- Measurement of upper limb function during daily life after stroke

Workshop on Robotics, Neuroscience and Learning - 2017, Dipartimento di Ingegneria dell'Informazione e Scienze Matematiche, Siena, Italy

- Sensor-based neurorehabilitation for stroke patients - monitoring and therapy

\section{Poster presentation:}

$4^{\text {th }}$ European Stroke Organisation Conference - ESOC 2018, Gothenburg, Sweden

- Encouraging real-world upper limb use after stroke by applying a tracking and feedback device

$2^{\text {nd }}$ International Congress on Neurorehabilitation and Neural Repair - NRNR 2017

Maastricht, The Netherlands

- Self-directed arm therapy at home after stroke with a sensor-based virtual reality training system

- Therapists perspectives on new technologies for upper limb function after stroke A focus group study

International Neurorehabilitation Symposium - INRS 2017, London, England

- Change of daily life arm usage after a virtual reality-based intervention for people with hemiplegic stroke

Zentrum für Neurowissenschaft Zürich - ZNZ-Symposium 2016, Zurich, Switzerland

- Self-directed arm therapy at home after stroke with a sensor-based virtual reality training system

International Conference on Neurorehabilitation - ICNR 2016, Segovia, Spain

- The evaluation of feedback modalities in stroke survivors

- Monitoring stroke patients in hospital and home environment 
